\title{
Synthesis of Silver(I) and Gold(I) Complexes Containing Enantiopure Pybox Ligands. First Assays on the Ag(I)-Catalyzed Asymmetric Addition of Alkynes to Imines
}

Gustavo M. Borrajo-Calleja, ${ }^{\mathrm{a}}$ Eire de Julián, ${ }^{\mathrm{a}}$ Esther Bayón, ${ }^{\mathrm{a}}$ Josefina Díez, ${ }^{\mathrm{a}}$ Elena Lastra, ${ }^{\mathrm{a}}$ Isabel Merino, ${ }^{\mathrm{b}}$ and M. Pilar Gamasa, ${ }^{\mathrm{a}}$ *

Departamento de Química Orgánica e Inorgánica-IUQOEM Instituto (Unidad Asociada al CSIC), Centro de Innovación en Química Avanzada (ORFEO-CINQA), Universidad de Oviedo, E-33006 Oviedo, Principado de Asturias, Spain.

\section{SUPPORTING INFORMATION.}

1. Experimental conditions for NMR experiments.

2. ORTEP drawing of cationic complexes 5, 9 and 11 (Figures $S 1-S 3$ ).

3. Characterization of the complexes $2,7,8$ and 11.

4. Low temperature ${ }^{1} \mathrm{H}$ and ${ }^{13} \mathrm{C}\left\{{ }^{1} \mathrm{H}\right\}$ NMR spectra for complexes $\mathbf{1 ,}, \mathbf{4}, \mathbf{6}, 10$ and 12.

5. Figure $S 4$. ${ }^{1} \mathrm{H}$ NMR of complex 10 in $\mathrm{CD}_{2} \mathrm{Cl}_{2}$ at (a) $298 \mathrm{~K}$, (b) $253 \mathrm{~K}$, (c) $213 \mathrm{~K}$ and (d) $193 \mathrm{~K}$.

6. Figure S5. (a) ${ }^{1} \mathrm{H} N M R$ of complex 12 in $\mathrm{CD}_{2} \mathrm{Cl}_{2}$ at $298 \mathrm{~K}$, (b) $253 \mathrm{~K}$, (c) $233 \mathrm{~K}$ and (d) $213 \mathrm{~K} ; \mathbf{L 2}, \mathbf{L 2}$ ' $=\left[\mathrm{Ag}_{2}(\text { indane-pybox })_{2}\right]\left[\mathrm{PF}_{6}\right]_{2} ; \mathbf{1 2}=\left[\mathrm{Ag}_{2}(\text { indane-pybox })_{3}\right]\left[\mathrm{PF}_{6}\right]_{2}$. (e) ${ }^{1} \mathrm{HNMR}$ of complex 4 in $\mathrm{CD}_{2} \mathrm{Cl}_{2}$ at $213 \mathrm{~K}$.

7. Figure $S 6 .{ }^{1} \mathrm{H}$ DOSY spectrum of complex 12 at $213 \mathrm{~K}$.

8. NMR spectra for complexes 1, 4, 6, 10 and 12, including ${ }^{1} \mathrm{H} \mathrm{NMR},{ }^{13} \mathrm{C} \mathrm{NMR},{ }^{31} \mathrm{P}$ NMR, ${ }^{19} \mathrm{~F}$ NMR, ${ }^{1} \mathrm{H}$ DOSY, ${ }^{19} \mathrm{~F}$ DOSY, COSY, ${ }^{1} \mathrm{H}^{13} \mathrm{C}$ HSQC, ${ }^{1} \mathrm{H}^{13} \mathrm{C} \mathrm{HMBC},{ }^{1} \mathrm{H}^{19} \mathrm{~F}$ HOESY, ROESY at room or variable temperature.

\section{Experimental conditions for NMR experiments}

NMR spectra were recorded on a Bruker AV 600 spectrometer operating at 600.15 $\left({ }^{1} \mathrm{H}\right), 150.91 \mathrm{MHz}\left({ }^{13} \mathrm{C}\right)$, using a $5 \mathrm{~mm}$ TBO BB- ${ }^{1} \mathrm{H} /{ }^{13} \mathrm{C} / \mathrm{D}$ direct probe with a z-gradient coil, or on a Bruker AV 400 spectrometer operating at $400.13\left({ }^{1} \mathrm{H}\right)$ and 100.61 
$\mathrm{MHz}\left({ }^{13} \mathrm{C}\right)$, using a $5 \mathrm{~mm}$ TBO ${ }^{1} \mathrm{H} / \mathrm{X}-\mathrm{BB} /{ }^{31} \mathrm{P} / \mathrm{D}$ direct probe with a z-gradient coil, or on a Bruker AV 300 spectrometer operating at $300.13\left({ }^{1} \mathrm{H}\right), 282.40\left({ }^{19} \mathrm{~F}\right)$ and $121.47 \mathrm{MHz}$ $\left({ }^{31} \mathrm{P}\right)$, using a $5 \mathrm{~mm} \mathrm{QNP}{ }^{1} \mathrm{H} /{ }^{13} \mathrm{C} /{ }^{19} \mathrm{~F} /{ }^{31} \mathrm{P} / \mathrm{D}$ probe with a z-gradient coil. The samples were prepared under argon atmosphere and dried, freshly distilled deuterated methylene chloride $\left(\mathrm{CD}_{2} \mathrm{Cl}_{2}\right)$ was used as solvent. All the experiments were acquired and processed with the TOPSPIN 2.1 Bruker NMR software. The COSY, ROESY, HSQC and $\mathrm{HMBC}$ experiments were recorded on the AV 600 spectrometer using the gradient enhanced versions of the pulse sequences. The variable temperature studies as well as the ${ }^{1} \mathrm{H}$-DOSY experiments at low temperature were performed on the AV600 spectrometer. The ${ }^{1} \mathrm{H}$ and ${ }^{19} \mathrm{~F}$-DOSY experiments at room temperature were performed on the AV400 spectrometer. A typical sample contained 3-4 mg of the corresponding silver complex dissolved in $0.5 \mathrm{ml}$ of deuterated solvent. On the AV 400 spectrometer, the ${ }^{19} \mathrm{~F}$ nucleus was tuned to a basic transmitter frequency of $376.45 \mathrm{MHz}$ through the ${ }^{1} \mathrm{H}$ channel of the probe and was detected through the X-BB preamplifier module. To the X-BB channel a D-stop filter and a ${ }^{13} \mathrm{Cstop} /{ }^{31}$ Ppass filter were added. For these conditions the 90 degree pulse was calibrated to 10.76 us for an attenuation level of 0 $\mathrm{dB}$. The gradient unit of the spectrometer produce magnetic field pulsed gradients in the $\mathrm{z}$-axis of $53.5 \mathrm{G} / \mathrm{cm}$. The gradient strength was calibrated using a $\mathrm{D}_{2} \mathrm{O}$ sample to obtain a diffusion coefficient of $1.90 \times 10^{-9} \mathrm{~m}^{2} / \mathrm{s}$ for HDO. During the DOSY experiments, the temperature was set to $298 \mathrm{~K}$ and maintained with an air flow of $535 \mathrm{l} / \mathrm{h}$. The experiments were acquired with the bipolar longitudinal eddy current delay pulse program (ledbpgps $2 s$ ) in Bruker software. The ${ }^{1} \mathrm{H}$ DOSY experiments were acquired with spinning of the sample to avoid convection effects and without spinning for the ${ }^{19} \mathrm{~F}$ DOSY measurements, since no differences were observed when spinning was applied. The diffusion time (D20) and the gradient duration (P30) were optimized with the ledbpgp 2 s $1 d$ sequence previously for each measurement to get a $1-5 \%$ of residual signal with the maximum strength, while observing a progressive decay of the signal intensities. For a typical ${ }^{1} \mathrm{H}$ or ${ }^{19} \mathrm{~F}$ - DOSY experiment, P30 was $0.9-1.2 \mathrm{~ms}$ and D20 was 100-200 ms. An eddy current delay (Te) of $5 \mathrm{~ms}$ and a spoil gradient (P19) of $0.8 \mathrm{~ms}$ were used. The pulse gradients were increased from 2 to $95 \%$ of the maximum strength in a linear ramp through 16 steps of $16 \mathrm{~K}$ data points each. For the ${ }^{1} \mathrm{H}$ DOSY experiments the size of the FID was $16 \mathrm{~K}$, the spectral width was set to $\mathrm{AQ}=2.018 \mathrm{~s}$, the number of scans were $16-32$ and a relaxation delay $\mathrm{D} 1=1 \mathrm{~s}$ was used. For the ${ }^{19} \mathrm{~F}$ DOSY experiments the size of the FID was $16 \mathrm{~K}$, the spectral width was set to AQ $=$ 
$1.356 \mathrm{~s}$, the number of scans were 32 and a relaxation delay D1 $=2 \mathrm{~s}$ was used. The direct dimension was zero-filled to $32 \mathrm{~K}$, fourier transformed and phase corrected. The diffusion dimension was processed with the standard Bruker dosy algorithm. The diffusion coefficients were measured in a logarithmic or lineal scale and the accuracy of the reported values are $\pm 0.01\left({ }^{1} \mathrm{H}\right.$-DOSY) and $\pm 0.02\left({ }^{19} \mathrm{~F}\right.$-DOSY $)$ in the logarithmic scale and $\pm 0.01 * 10^{-9}$ in the linear scale $\left({ }^{1} \mathrm{H}\right.$-DOSY). The ${ }^{1} \mathrm{H}^{19} \mathrm{~F}$ HOESY experiments were performed on the AV300 spectrometer and acquired in the phase-sensitive mode with the hoesyph pulse sequence. Selected parameters for this experiment were sweep width ca $3000 \mathrm{~Hz}, 1024$ x 128 data set, number of scans 32, mixing time D8 = $2.1 \mathrm{~s}$, relaxation delay D1 $=1.8 \mathrm{~s}$. Data were processed using zero filling in the F1 domain to 1024 data points and shifted squared sine-bell apodization of factor 2 in both dimensions.

\section{ORTEP drawing of cationic complexes 5, 9 and 11}

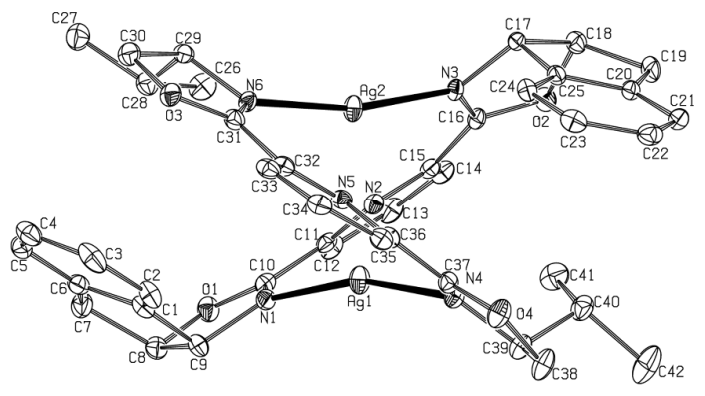

Figure $\boldsymbol{S} 1$. ORTEP drawing of cationic complex 5 showing atom-labeling scheme. Thermal ellipsoids are shown at the $20 \%$ probability level. Hydrogen atoms and the $\mathrm{OTf}^{-}$anions are omitted for clarity.

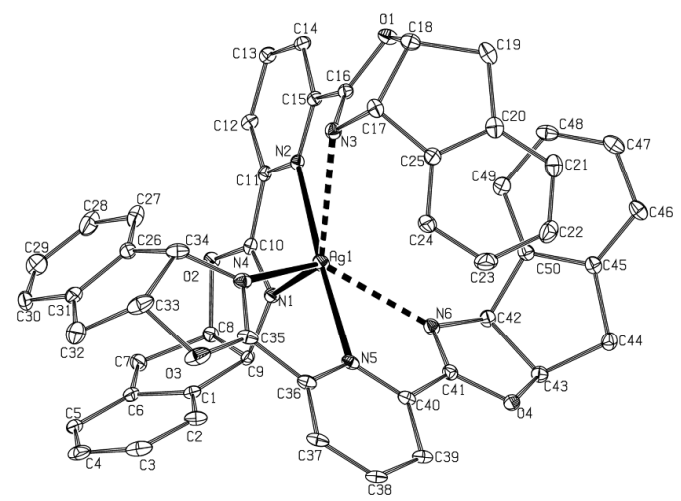

Figure S2. ORTEP drawing of complex 9 showing atom-labeling scheme. Thermal ellipsoids are shown at the $20 \%$ probability level. Solvent molecules, hydrogen atoms and the $\mathrm{BF}_{4}{ }^{-}$anion are omitted for clarity. 


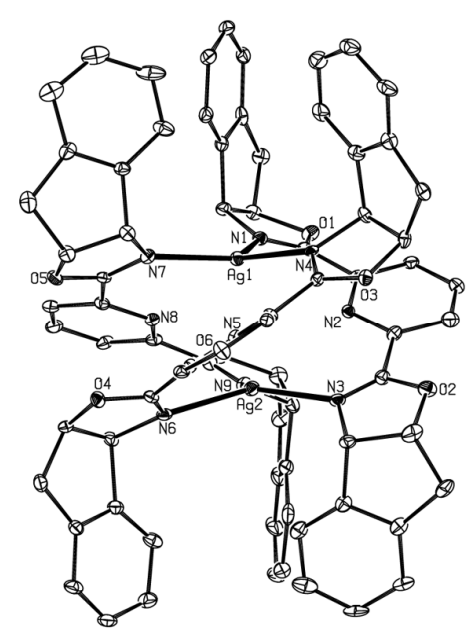

Figure S3. ORTEP drawing of cationic complex 11 showing atom-labeling scheme. Thermal ellipsoids are shown at the $10 \%$ probability level. Solvent molecules, hydrogen atoms and the $\mathrm{SbF}_{6}{ }^{-}$anions are omitted for clarity.

Characterization of the dinuclear complex $\left[\mathrm{Ag}_{2}\left\{(S, S)-{ }^{i} \mathrm{Pr}-\mathrm{pybox}_{2}\right]\left[\mathrm{PF}_{6}\right]_{2}\right.$ (2). Color: pale brown. Yield: $50 \%(0.055 \mathrm{~g}) . \Lambda_{\mathrm{M}}=258 \mathrm{~S} \mathrm{~cm}^{2} \mathrm{~mol}^{-1}$ (acetone, $\left.293 \mathrm{~K}\right)$. IR (KBr): $v\left(\mathrm{PF}_{6}^{-}\right) 830(\mathrm{vs}) \mathrm{cm}^{-1} .{ }^{31} \mathrm{P}$ NMR (121.5 MHz, $\left.\mathrm{CDCl}_{3}, 298 \mathrm{~K}\right):-144.6\left(\mathrm{sp}, J_{\mathrm{PF}}=714.2\right.$ $\left.\mathrm{Hz}, \mathrm{PF}_{6}{ }^{-}\right) .{ }^{1} \mathrm{H}$ NMR $\left(400.13 \mathrm{MHz}, \mathrm{Me}_{2} \mathrm{CO}-\mathrm{d}_{6}, 298 \mathrm{~K}\right): \delta 8.57\left(\mathrm{t}, J_{\mathrm{HH}}=7.2 \mathrm{~Hz}, 2 \mathrm{H}, \mathrm{H}^{4}\right.$ $\left.\mathrm{C}_{5} \mathrm{H}_{3} \mathrm{~N}\right), 8.46\left(\mathrm{~d}, J_{\mathrm{HH}}=7.2 \mathrm{~Hz}, 4 \mathrm{H}, \mathrm{H}^{3,5} \mathrm{C}_{5} \mathrm{H}_{3} \mathrm{~N}\right), 5.04\left(\mathrm{~m}, 4 \mathrm{H}, \mathrm{OCH}_{2}\right), 4.73(\mathrm{~m}, 4 \mathrm{H}$, $\left.\mathrm{OCH}_{2}\right), 4.37(\mathrm{~m}, 4 \mathrm{H}, \mathrm{CH} P r), 1.90\left(\mathrm{~m}, 4 \mathrm{H}, \mathrm{CHMe}\right.$ ), $0.92\left(\mathrm{~d}, J_{\mathrm{HH}}=7.2 \mathrm{~Hz}, 12 \mathrm{H}\right.$, $\left.\mathrm{CHMe} e_{2}\right), 0.87\left(\mathrm{~d}, J_{\mathrm{HH}}=6.8 \mathrm{~Hz}, 12 \mathrm{H}, \mathrm{CHMe} 2\right) \mathrm{ppm} .{ }^{13} \mathrm{C}\left\{{ }^{1} \mathrm{H}\right\}$ NMR (100.61 MHz, $\left.\mathrm{Me}_{2} \mathrm{CO}-\mathrm{d}_{6}, 298 \mathrm{~K}\right): \delta 166.4(\mathrm{~s}, \mathrm{OCN}), 144.0\left(\mathrm{~s}, \mathrm{C}^{2,6} \mathrm{C}_{5} \mathrm{H}_{3} \mathrm{~N}\right), 141.8\left(\mathrm{~s}, \mathrm{C}^{4} \mathrm{C}_{5} \mathrm{H}_{3} \mathrm{~N}\right), 128.3$ $\left(\mathrm{s}, \mathrm{C}^{3,5} \mathrm{C}_{5} \mathrm{H}_{3} \mathrm{~N}\right), 73.4\left(\mathrm{~s}, \mathrm{OCH}_{2}\right), 71.1\left(\mathrm{~s}, \mathrm{CH}^{i} \mathrm{Pr}\right), 31.5\left(\mathrm{~s}, \mathrm{CHMe}_{2}\right), 17.7,16.6(2 s$, $\mathrm{CHMe}$ ) ppm. Anal. Calcd for $\mathrm{C}_{34} \mathrm{H}_{46} \mathrm{~N}_{6} \mathrm{~F}_{12} \mathrm{O}_{4} \mathrm{P}_{2} \mathrm{Ag}_{2}$ (1108.44): C, 36.84; H, 4.18; N, 7.58. Found: C, 36.79; H, 4.22; N, 7.37.

Characterization of mononuclear complexes $\left[A g\left\{(S, S)-{ }^{i} P r-p y b o x\right\}_{2}\right][X]\left(X=P_{6}(7)\right.$, $\left.\mathrm{BF}_{4}(8)\right)$.

Complex 7. Color: cream. Yield: $83 \%(0.106 \mathrm{~g}) . \Lambda_{\mathrm{M}}=114 \mathrm{~S} \mathrm{~cm}^{2} \mathrm{~mol}^{-1}$ (acetone, 293 K). IR (KBr): v( $\left.\mathrm{PF}_{6}{ }^{-}\right) 839$ (vs) $\mathrm{cm}^{-1} .{ }^{1} \mathrm{H} \mathrm{NMR}\left(400.13 \mathrm{MHz}, \mathrm{CD}_{2} \mathrm{Cl}_{2}, 298 \mathrm{~K}\right.$ ): $\delta 8.19$ (s, $6 \mathrm{H}, \mathrm{H}^{3,4,5} \mathrm{C}_{5} \mathrm{H}_{3} \mathrm{~N}$ ), $4.62\left(\mathrm{pt}, J_{\mathrm{HH}}=9.2 \mathrm{~Hz}, 4 \mathrm{H}, \mathrm{OCH}_{2}\right.$ ), 4.22 (pt, $J_{\mathrm{HH}}=8.8 \mathrm{~Hz} 4 \mathrm{H}$, 
$\left.\mathrm{OCH}_{2}\right), 3.92(\mathrm{~m}, 4 \mathrm{H}, \mathrm{CH} P r), 1.65\left(\mathrm{~m}, 4 \mathrm{H}, \mathrm{CHMe}\right.$ ), $0.82\left(\mathrm{~d}, J_{\mathrm{HH}}=6.8 \mathrm{~Hz}, 12 \mathrm{H}\right.$, $\left.\mathrm{CHMe} e_{2}\right), 0.77\left(\mathrm{~d}, J_{\mathrm{HH}}=6.8 \mathrm{~Hz}, 12 \mathrm{H}, \mathrm{CHMe} 2\right) \mathrm{ppm} .{ }^{13} \mathrm{C}\left\{{ }^{1} \mathrm{H}\right\}$ NMR $(100.61 \mathrm{MHz}$,

$\left.\mathrm{CD}_{2} \mathrm{Cl}_{2}, 298 \mathrm{~K}\right): \delta 162.2(\mathrm{~s}, \mathrm{OCN}), 144.2\left(\mathrm{~s}, \mathrm{C}^{2,6} \mathrm{C}_{5} \mathrm{H}_{3} \mathrm{~N}\right), 139.7\left(\mathrm{~s}, \mathrm{C}^{3,5} \mathrm{C}_{5} \mathrm{H}_{3} \mathrm{~N}\right), 125.7$ $\left(\mathrm{s}, \mathrm{C}^{4} \mathrm{C}_{5} \mathrm{H}_{3} \mathrm{~N}\right), 72.6\left(\mathrm{~s}, \mathrm{OCH}_{2}\right), 72.2\left(\mathrm{~s}, \mathrm{CH}^{i} \mathrm{Pr}\right), 32.6\left(\mathrm{~s}, \mathrm{CHMe}_{2}\right), 18.3,18.0$ (2s, $\left.\mathrm{CH} \mathrm{Me}_{2}\right) \mathrm{ppm}$.

Complex 8. Color: cream. Yield: $82 \%(0.980 \mathrm{~g}) . \Lambda_{\mathrm{M}}=109 \mathrm{~S} \mathrm{~cm}^{2} \mathrm{~mol}^{-1}$ (acetone, 293 $\mathrm{K})$. IR ( $\mathrm{KBr}): v\left(\mathrm{BF}_{4}{ }^{-}\right) 1051(\mathrm{vs}) \mathrm{cm}^{-1} .{ }^{1} \mathrm{H} \mathrm{NMR}\left(400.13 \mathrm{MHz}, \mathrm{CD}_{2} \mathrm{Cl}_{2}, 298 \mathrm{~K}\right): \delta 8.20$ $\left(\mathrm{s}, 6 \mathrm{H}, \mathrm{H}^{3,4,5} \mathrm{C}_{5} \mathrm{H}_{3} \mathrm{~N}\right), 4.63\left(\mathrm{pt}, J_{\mathrm{HH}}=9.2 \mathrm{~Hz}, 4 \mathrm{H}, \mathrm{OCH}_{2}\right), 4.22\left(\mathrm{pt}, J_{\mathrm{HH}}=9.2 \mathrm{~Hz} 4 \mathrm{H}\right.$, $\left.\mathrm{OCH}_{2}\right), 3.92(\mathrm{~m}, 4 \mathrm{H}, \mathrm{CH} \mathrm{Pr}), 1.65\left(\mathrm{~m}, 4 \mathrm{H}, \mathrm{C} H \mathrm{Me}_{2}\right), 0.82\left(\mathrm{~d}, J_{\mathrm{HH}}=6.8 \mathrm{~Hz}, 12 \mathrm{H}\right.$, $\mathrm{CHMe} 2), 0.78\left(\mathrm{~d}, J_{\mathrm{HH}}=6.8 \mathrm{~Hz}, 12 \mathrm{H}, \mathrm{CHMe} 2\right) \mathrm{ppm} .{ }^{13} \mathrm{C}\left\{{ }^{1} \mathrm{H}\right\}$ NMR $(100.61 \mathrm{MHz}$, $\left.\mathrm{CD}_{2} \mathrm{Cl}_{2}, 298 \mathrm{~K}\right): \delta 162.2(\mathrm{~s}, \mathrm{OCN}), 144.2\left(\mathrm{~s}, \mathrm{C}^{2,6} \mathrm{C}_{5} \mathrm{H}_{3} \mathrm{~N}\right), 139.8\left(\mathrm{~s}, \mathrm{C}^{3,5} \mathrm{C}_{5} \mathrm{H}_{3} \mathrm{~N}\right), 125.8$ $\left(\mathrm{s}, \mathrm{C}^{4} \mathrm{C}_{5} \mathrm{H}_{3} \mathrm{~N}\right), 72.7\left(\mathrm{~s}, \mathrm{OCH}_{2}\right), 72.2\left(\mathrm{~s}, \mathrm{CH}^{i} \mathrm{Pr}\right), 32.6\left(\mathrm{~s}, \mathrm{CHMe}_{2}\right), 18.3,18.0$ (2s, $\left.\mathrm{CH} \mathrm{Me}_{2}\right) \mathrm{ppm}$.

\section{Characterization of dinuclear complex [Ag $\left.\left\{\left(3 a S, 3 a^{\prime} S, 8 a R, 8 a^{\prime} R\right) \text {-indane-pybox }\right\}_{3}\right] l$}

$\left.\boldsymbol{S b F}_{6}\right]_{2}$ (11). Color: cream. Yield: $42 \%(0.039 \mathrm{~g}) . \Lambda_{\mathrm{M}}=277 \mathrm{~S} \mathrm{~cm}^{2} \mathrm{~mol}^{-1}$ (acetone, 293 K). IR (KBr): v( $\left.\mathrm{SbF}_{6}{ }^{-}\right) 658$ (vs). ${ }^{1} \mathrm{H}$ NMR (400.13 MHz, $\left.\mathrm{Me}_{2} \mathrm{CO}-\mathrm{d}_{6}, 298 \mathrm{~K}\right): \delta 8.48$ (br s, 9H, $\mathrm{H}^{3,4,5} \mathrm{C}_{5} \mathrm{H}_{3} \mathrm{~N}$ ), 7.35 (br s, $12 \mathrm{H}, \mathrm{CH}_{\text {arom }}$ ), 6.77 (br s, 6H, $\mathrm{CH}_{\text {arom }}$ ), 6.41 (br s, $6 \mathrm{H}$, $\mathrm{CH}_{\text {arom}}$ ), 5.75 (m, 6H, OCH), 4.39 (br s, 6H, NCH), 3.59 (br s, 12H, $\mathrm{CH}_{2}$ ) ppm. ${ }^{13} \mathrm{C}\left\{{ }^{1} \mathrm{H}\right\}$ NMR $\left(100.61 \mathrm{MHz}, \mathrm{Me}_{2} \mathrm{CO}-\mathrm{d}_{6}, 298 \mathrm{~K}\right): \delta 164.3(\mathrm{~s}, \mathrm{OCN}), 144.9\left(\mathrm{~s}, \mathrm{C}^{2,6}\right.$ $\left.\mathrm{C}_{5} \mathrm{H}_{3} \mathrm{~N}\right), 140.8\left(\mathrm{~s}, \mathrm{C}^{4} \mathrm{C}_{5} \mathrm{H}_{3} \mathrm{~N}\right), 140.0,139.4\left(2 s, \mathrm{C}_{\text {arom }}\right), 129.2\left(\mathrm{~s}, \mathrm{CH}_{\text {arom}}\right), 128.8\left(\mathrm{~s}, \mathrm{C}^{3,5}\right.$ $\left.\mathrm{C}_{5} \mathrm{H}_{3} \mathrm{~N}\right), 127.9,125.8,125.1$ ( $\left.3 \mathrm{~s}, \mathrm{CH}_{\text {arom }}\right), 86.4(\mathrm{~s}, \mathrm{OCH}), 76.1(\mathrm{~s}, \mathrm{NCH}), 39.4\left(\mathrm{~s}, \mathrm{CH}_{2}\right)$ ppm. Anal. Calcd for $\mathrm{C}_{75} \mathrm{H}_{57} \mathrm{~N}_{9} \mathrm{Sb}_{2} \mathrm{~F}_{12} \mathrm{O}_{6} \mathrm{Ag}_{2}$ (1867.58): C, 48.24; H, 3.08; N, 6.75. Found: C, 48.35; H, 3.48; N, 6.49 .

\section{Low temperature ${ }^{1} \mathrm{H}$ and $\left.{ }^{13} C_{\{}{ }^{1} H\right\}$ NMR spectra for complexes 1, 4, 6, 10 and 12}

Complex 1. ${ }^{1} \mathrm{H}$ NMR (400.13 MHz, $\left.\mathrm{CD}_{2} \mathrm{Cl}_{2}, 183 \mathrm{~K}\right): \mathbf{M}: \mathbf{m}=80: 20 ; \delta 8.30(\mathrm{~s}, 6 \mathrm{H}$, $\left.\mathrm{C}_{5} \mathrm{H}_{3} \mathrm{~N} \mathrm{M}, \mathbf{m}\right), 4.94\left(\mathrm{t}, 4 \mathrm{H}, J_{\mathrm{HH}}=9.5 \mathrm{~Hz}, \mathrm{OCH}_{2} \mathbf{M}, \mathbf{m}\right), 4.57\left(\mathrm{t}, J_{\mathrm{HH}}=8.6 \mathrm{~Hz}, 4 \mathrm{H}, \mathrm{OCH}_{2}\right.$ m), $4.46\left(\mathrm{t}, J_{\mathrm{HH}}=8.6 \mathrm{~Hz}, 4 \mathrm{H}, \mathrm{OCH}_{2} \mathbf{M}\right), 4.28\left(\mathrm{~m}, 4 \mathrm{H}, \mathrm{C} H^{i} \operatorname{Pr} \mathbf{m}\right), 4.18\left(\mathrm{q}, J_{\mathrm{HH}}=9.5 \mathrm{~Hz}\right.$, 4H, CH $\left.H^{i} \operatorname{Pr} \mathbf{M}\right), 1.93$ (m, 4H, CHMe 2 m), 1.56 (m, 4H, CHMe 2 M), 0.79 (m, 12H, $\left.\mathrm{CHMe}_{2} \mathbf{M}, \mathbf{m}\right) \mathrm{ppm} .{ }^{13} \mathrm{C}\left\{{ }^{1} \mathrm{H}\right\} \mathrm{NMR}\left(100.61 \mathrm{MHz}, \mathrm{CD}_{2} \mathrm{Cl}_{2}, 183 \mathrm{~K}\right): \delta 165.8(\mathrm{C}, \mathrm{OCN}$ m), $165.7(\mathrm{C}, \mathrm{OCN} \mathbf{M}), 143.6\left(\mathrm{C}, \mathrm{C}^{2,6} \mathrm{C}_{5} \mathrm{H}_{3} \mathrm{~N} \mathrm{M}\right), 143.5\left(\mathrm{C}, \mathrm{C}^{2,6} \mathrm{C}_{5} \mathrm{H}_{3} \mathrm{~N}\right.$ m $), 141.4(\mathrm{CH}$, $\left.\mathrm{C}^{4} \mathrm{C}_{5} \mathrm{H}_{3} \mathrm{~N}\right), 129.1\left(\mathrm{CH}, \mathrm{C}^{3,5} \mathrm{C}_{5} \mathrm{H}_{3} \mathrm{~N} \mathrm{M}\right), 128.7\left(\mathrm{CH}, \mathrm{C}^{3,5} \mathrm{C}_{5} \mathrm{H}_{3} \mathrm{~N} \mathbf{m}\right), 120.5\left(\mathrm{C}^{1} J_{\mathrm{CF}}=\right.$ $320 \mathrm{~Hz}), 74.9\left(\mathrm{CH}_{2}, \mathrm{OCH}_{2} \mathbf{M}\right), 72.3\left(\mathrm{CH}_{2}, \mathrm{OCH}_{2} \mathbf{m}\right), 72.1\left(\mathrm{CH}, \mathrm{CH}^{i} \operatorname{Pr} \mathbf{M}\right), 70.0(\mathrm{CH}$, 
$\left.\mathrm{CH}^{i} \mathrm{Pr} \mathbf{m}\right), 33.8\left(\mathrm{CH}, \mathrm{CHMe}_{2} \mathrm{M}\right), 31.5\left(\mathrm{CH}, \mathrm{CHMe}_{2} \mathbf{m}\right), 19.2,18.9\left(\mathrm{CH}_{3}, \mathrm{CHMe}_{2} \mathbf{M}\right)$ 18.2, $15.7\left(\mathrm{CH}_{3}, \mathrm{CHMe} 2 \mathrm{~m}\right)$ ppm. ${ }^{19} \mathrm{~F}\left\{{ }^{1} \mathrm{H}\right\} \mathrm{NMR}\left(376.5 \mathrm{MHz}, \mathrm{CD}_{2} \mathrm{Cl}_{2}, 183 \mathrm{~K}\right)$ : -79.4 ppm.

Complex 4. ${ }^{1} \mathrm{H}$ NMR (600.15 MHz, $\left.\mathrm{CD}_{2} \mathrm{Cl}_{2}, 213 \mathrm{~K}\right): \mathbf{M}: \mathbf{m}=85: 15 ; \delta 8.45(\mathrm{~m}, 6 \mathrm{H}$, $\left.\mathrm{C}_{5} \mathrm{H}_{3} \mathrm{~N} \mathrm{M}\right), 8.35$ (bs, 6H, $\mathrm{C}_{5} \mathrm{H}_{3} \mathrm{~N} \mathrm{~m}$ ), 7.40 (d, 4H, $\left.J=7.5 \mathrm{~Hz}, \mathrm{CH}_{\text {arom }} \mathbf{m}\right), 7.36$ (t, 4H, $J$ $\left.=7.6 \mathrm{~Hz}, \mathrm{CH}_{\text {arom }} \mathbf{m}\right), 7.22\left(\mathrm{t}, 4 \mathrm{H}, J=7.5 \mathrm{~Hz}, \mathrm{CH}_{\text {arom }} \mathbf{M}\right), 7.06(\mathrm{~d}, 4 \mathrm{H}, J=7.6 \mathrm{~Hz}$, $\left.\mathrm{CH}_{\text {arom }} \mathbf{M}\right), 6.92\left(\mathrm{~d}, 4 \mathrm{H}, J=7.7 \mathrm{~Hz}, \mathrm{CH}_{\text {arom }} \mathbf{m}\right), 6.89$ (t, $\left.4 \mathrm{H}, J=7.6 \mathrm{~Hz}, \mathrm{CH}_{\text {arom }} \mathbf{m}\right), 6.86$ (t, $\left.4 \mathrm{H}, J=7.5 \mathrm{~Hz}, \mathrm{CH}_{\text {arom }} \mathbf{M}\right), 6.63\left(\mathrm{~d}, 4 \mathrm{H}, J=7.5 \mathrm{~Hz}, \mathrm{CH}_{\text {arom }} \mathbf{M}\right), 6.17$ (bt, $J=7.4 \mathrm{~Hz}$, 4H, OCH m), 5.84 (ddd, $J=9.1,7.3,2.1 \mathrm{~Hz}, 4 \mathrm{H}, \mathrm{OCH} \mathbf{M}), 5.80(\mathrm{~d}, J=8.0 \mathrm{~Hz}, 4 \mathrm{H}$, $\mathrm{NCH} \mathbf{m}), 5.73(\mathrm{~d}, J=9.1 \mathrm{~Hz}, 4 \mathrm{H}, \mathrm{NCH} \mathbf{M}), 3.68\left(\mathrm{dd}, J=18.2,6.2,1 \mathrm{H}, \mathrm{CH}_{2} \mathbf{m}\right), 3.61$ $\left(\mathrm{d}, J=18.2,1 \mathrm{H}, \mathrm{CH}_{2} \mathbf{m}\right), 3.41\left(\mathrm{dd}, J=17.6,7.3,1 \mathrm{H}, \mathrm{CH}_{2} \mathbf{M}\right), 3.09$ (d, $J=17.6,1 \mathrm{H}$, $\left.\mathrm{CH}_{2} \mathbf{M}\right)$.

Complex 6. ${ }^{1} \mathrm{H}$ NMR (400.13 MHz, $\left.\mathrm{CD}_{2} \mathrm{Cl}_{2}, 193 \mathrm{~K}\right): \delta 8.15\left(\mathrm{~m}, 6 \mathrm{H}, \mathrm{H}^{3,4,5} \mathrm{C}_{5} \mathrm{H}_{3} \mathrm{~N}\right)$, $4.61\left(\mathrm{t}, J_{\mathrm{HH}}=9.3 \mathrm{~Hz}, 4 \mathrm{H}, \mathrm{OCH}_{2}\right), 4.15\left(\mathrm{t}, J_{\mathrm{HH}}=9.1 \mathrm{~Hz} 4 \mathrm{H}, \mathrm{OCH}_{2}\right), 3.83\left(\mathrm{q}, J_{\mathrm{HH}}=9.2\right.$ $\left.\mathrm{Hz}, 4 \mathrm{H}, \mathrm{C} H^{i} \mathrm{Pr}\right), 1.55\left(\mathrm{~m}, 4 \mathrm{H}, \mathrm{C} H \mathrm{Me}_{2}\right), 0.90\left(\mathrm{t}, J_{\mathrm{HH}}=5.0 \mathrm{~Hz}, 12 \mathrm{H}, \mathrm{CHMe}\right) \mathrm{ppm}$. ${ }^{13} \mathrm{C}\left\{{ }^{1} \mathrm{H}\right\}$ NMR $\left(100.61 \mathrm{MHz}, \mathrm{CD}_{2} \mathrm{Cl}_{2}, 193 \mathrm{~K}\right): \delta 161.8(\mathrm{C}, \mathrm{OCN}), 143.6\left(\mathrm{C}, \mathrm{C}^{2,6}\right.$ $\left.\mathrm{C}_{5} \mathrm{H}_{3} \mathrm{~N}\right), 140.1\left(\mathrm{CH}, \mathrm{C}^{3,5} \mathrm{C}_{5} \mathrm{H}_{3} \mathrm{~N}\right), 125.7\left(\mathrm{CH}, \mathrm{C}^{4} \mathrm{C}_{5} \mathrm{H}_{3} \mathrm{~N}\right), 73.0\left(\mathrm{CH}_{2}, \mathrm{OCH}_{2}\right), 71.9(\mathrm{CH}$, $\left.\left.\mathrm{CH}^{i} \mathrm{Pr}\right), 33.0\left(\mathrm{CH}, \mathrm{CHMe}_{2}\right), 18.8,18.2\left(\mathrm{CH}_{3}, \mathrm{CHMe}\right)_{2}\right) \mathrm{ppm}$.

Complex 10. ${ }^{1} \mathrm{H}$ NMR $\left(600.15 \mathrm{MHz}, \mathrm{CD}_{2} \mathrm{Cl}_{2}, 213 \mathrm{~K}\right): \mathbf{1 0}: \mathbf{4}=82: 18 ; \delta 8.45(\mathrm{~m}, 6 \mathrm{H}$, $\left.\mathrm{C}_{5} \mathrm{H}_{3} \mathrm{~N}, 4\right), 8.30\left(\mathrm{~m}, 9 \mathrm{H}, \mathrm{C}_{5} \mathrm{H}_{3} \mathrm{~N}, \mathbf{1 0}\right), 7.32\left(\mathrm{~m}, 12 \mathrm{H}, \mathrm{CH}_{\text {arom }}, \mathbf{1 0}\right), 7.22$ (t, J=7.5 Hz, 4H, $\left.\mathrm{CH}_{\text {arom }}, 4\right), 7.05\left(\mathrm{~d}, J=7.5 \mathrm{~Hz}, 4 \mathrm{H}, \mathrm{CH}_{\text {arom }}, 4\right), 6.86\left(\mathrm{t}, J=7.5 \mathrm{~Hz}, 4 \mathrm{H}, \mathrm{CH}_{\text {arom }}, 4\right), 6.73$ $\left(\mathrm{m}, 12 \mathrm{H}, \mathrm{CH}_{\text {arom }}, \mathbf{1 0}\right), 6.63\left(\mathrm{~d}, J=7.5 \mathrm{~Hz}, 4 \mathrm{H}, \mathrm{CH}_{\text {arom }}, 4\right), 6.35$ (d, $J=7.6 \mathrm{~Hz}, 6 \mathrm{H}$, $\left.\mathrm{CH}_{\text {arom }}, 10\right), 5.84$ (ddd, $\left.J=8.8,7.6,1.7 \mathrm{~Hz}, 4 \mathrm{H}, \mathrm{OCH}, 4\right), 5.73$ (d, $J=8.8 \mathrm{~Hz}, 4 \mathrm{H}, \mathrm{NCH}$, 4), $5.54(\mathrm{ddd}, J=8.3,6.8,2.0 \mathrm{~Hz}, 6 \mathrm{H}, \mathrm{OCH}, 10), 4.01(\mathrm{~d}, J=8.3 \mathrm{~Hz}, 6 \mathrm{H}, \mathrm{NCH}, 10$ ), $3.57\left(\mathrm{dd}, J=18.6,6.8 \mathrm{~Hz}, 6 \mathrm{H}, \mathrm{CH}_{2}, \mathbf{1 0}\right), 3.52$ (d, $\left.J=18.6 \mathrm{~Hz}, 6 \mathrm{H}, \mathrm{CH}_{2}, \mathbf{1 0}\right), 3.39$ (dd, $J$ $\left.=17.2,7.6 \mathrm{~Hz}, 4 \mathrm{H}, \mathrm{CH}_{2}, 4\right), 3.09\left(\mathrm{~d}, J=17.2 \mathrm{~Hz}, 4 \mathrm{H}, \mathrm{CH}_{2}, 4\right) .{ }^{13} \mathrm{C}\left\{{ }^{1} \mathrm{H}\right\} \mathrm{NMR}(150.91$ $\left.\mathrm{MHz}, \mathrm{CD}_{2} \mathrm{Cl}_{2}, 213 \mathrm{~K}\right): \delta 163.9(\mathrm{C}, \mathrm{OCN}, 10), 165.2(\mathrm{C}, \mathrm{OCN}, 4), 144.4\left(\mathrm{C}, \mathrm{C}^{2,6} \mathrm{C}_{5} \mathrm{H}_{3} \mathrm{~N}\right.$, 10), $144.3\left(\mathrm{C}, \mathrm{C}^{2,6} \mathrm{C}_{5} \mathrm{H}_{3} \mathrm{~N}, 4\right), 140.9\left(\mathrm{CH}, \mathrm{C}^{4} \mathrm{C}_{5} \mathrm{H}_{3} \mathrm{~N}, \mathbf{1 0}\right), 141.5\left(\mathrm{CH}, \mathrm{C}^{4} \mathrm{C}_{5} \mathrm{H}_{3} \mathrm{~N}, 4\right)$, 139.9, 138.7 (2 $\left.\mathrm{C}_{\text {arom }}, \mathbf{1 0}\right), 139.1,138.1$ (2 $\left.\mathrm{C}_{\text {arom }}, \mathbf{4}\right), 129.6\left(\mathrm{CH}_{\text {arom }}, \mathbf{1 0}\right), 128.8\left(\mathrm{CH}_{\text {arom, }}\right.$ 4), $128.6\left(\mathrm{CH}, \mathrm{C}^{3,5} \mathrm{C}_{5} \mathrm{H}_{3} \mathrm{~N}, \mathbf{1 0}\right), 128.5\left(\mathrm{CH}, \mathrm{C}^{3,5} \mathrm{C}_{5} \mathrm{H}_{3} \mathrm{~N}, 4\right), 127.8\left(\mathrm{CH}_{\text {arom }}, \mathbf{1 0}\right), 127.0$, $126.9\left(2 \mathrm{CH}_{\text {arom }}, 4\right), 126.1\left(\mathrm{CH}_{\text {arom }}, \mathbf{1 0}\right), 124.9\left(\mathrm{CH}_{\text {arom }}, \mathbf{1 0}\right), 123.5\left(\mathrm{CH}_{\text {arom }}, 4\right), 120.5(\mathrm{C}$, 
$\left.{ }^{1} J_{\mathrm{CF}}=319 \mathrm{~Hz}\right), 86.5(\mathrm{CH}, \mathrm{OCH}, \mathbf{1 0}), 88.3(\mathrm{CH}, \mathrm{OCH}, 4), 75.9(\mathrm{CH}, \mathrm{NCH}, \mathbf{1 0}), 75.3$ (CH, NCH, 4), $39.7\left(\mathrm{CH}_{2}, \mathrm{CH}_{2} \mathrm{C}_{\text {arom }}, 10\right), 38.6\left(\mathrm{CH}_{2}, \mathrm{CH}_{2} \mathrm{C}_{\text {arom }}, 4\right)$.

Complex 12. ${ }^{1} \mathrm{H}$ NMR (600.15 MHz, $\left.\mathrm{CD}_{2} \mathrm{Cl}_{2}, 213 \mathrm{~K}\right): 12: \mathbf{L 2}: \mathbf{L 2}{ }^{\prime}=25: 51: 49 ; \delta$ $8.47\left(\mathrm{~m}, 6 \mathrm{H}, \mathrm{C}_{5} \mathrm{H}_{3} \mathrm{~N}, \mathbf{L 2}\right), 8.38\left(\mathrm{~m}, 6 \mathrm{H}, \mathrm{C}_{5} \mathrm{H}_{3} \mathrm{~N}, \mathbf{L 2}\right.$ ') 8.27 (m, 9H, $\left.\mathrm{C}_{5} \mathrm{H}_{3} \mathrm{~N}, \mathbf{1 2}\right), 7.42$ (d, $J=7.42 \mathrm{~Hz}, \mathrm{CH}_{\text {arom }}, 4 \mathrm{H}, \mathbf{L 2}$ '), 7.36 (t, $J=7.4 \mathrm{~Hz}, \mathrm{CH}_{\text {arom, }}$ 4H, L2'), 7.33 (m, 12H, $\left.\mathrm{CH}_{\text {arom }}, \mathbf{1 2}\right), 7.25$ (t, $\left.J=7.3 \mathrm{~Hz}, \mathrm{CH}_{\text {arom }}, 4 \mathrm{H}, \mathbf{L 2}\right), 7.08$ (d, $\left.J=7.3 \mathrm{~Hz}, \mathrm{CH}_{\text {arom }}, 4 \mathrm{H}, \mathbf{L 2}\right)$, 6.87 (m, 12H, $\mathrm{CH}_{\text {arom }}$ L2', L2', L2), 6.73 (m, 6H, $\mathrm{CH}_{\text {arom }}, \mathbf{1 2}$ ), 6.59 (d, J=7.1 Hz, 4H, $\mathrm{CH}_{\text {arom }}, \mathbf{L 2}$ ), 6.35 (d, $\left.J=7.7 \mathrm{~Hz}, 6 \mathrm{H}, \mathrm{CH}_{\text {arom }}, 12\right), 6.17$ (t, $J=7.5 \mathrm{~Hz}, 4 \mathrm{H}, \mathrm{OCH}, \mathbf{L 2}$ '), 5.85 (t, $J=8.4 \mathrm{~Hz}, 4 \mathrm{H}, \mathrm{OCH}, \mathbf{L 2}), 5.76$ (d, $J=8.1 \mathrm{~Hz}, 4 \mathrm{H}, \mathrm{NCH}, \mathbf{L 2}$ '), 5.85 (d, $J=9.0$ Hz, 4H, NCH, L2), 5.53 (ddd, $J=8.3,6.7,1.9 \mathrm{~Hz}, 6 \mathrm{H}, \mathrm{OCH}, 12), 4.06$ (d, $J=8.3 \mathrm{~Hz}$, 6H, NCH, 12), 3.68 (dd, $J=18.6,6.4 \mathrm{~Hz}, 4 \mathrm{H}, \mathrm{CH}_{2}, \mathbf{L 2}$ '), 3.61 (d, $J=18.6, \mathrm{~Hz}, 4 \mathrm{H}$, $\mathrm{CH}_{2}, \mathbf{L} 2$ '), 3.55 (dd, $\left.J=18.6,6.7 \mathrm{~Hz}, 6 \mathrm{H}, \mathrm{CH}_{2}, 12\right), 3.50$ (d, $J=18.6, \mathrm{~Hz}, 6 \mathrm{H}, \mathrm{CH}_{2}, 12$ ), $3.40\left(\mathrm{dd}, J=17.9,7.9 \mathrm{~Hz}, 4 \mathrm{H}, \mathrm{CH}_{2}, \mathbf{L 2}\right), 3.09$ (d, $\left.J=17.9 \mathrm{~Hz}, 4 \mathrm{H}, \mathrm{CH}_{2}, \mathbf{L 2}\right) .{ }^{13} \mathrm{C}$ $\left\{{ }^{1} \mathrm{H}\right\} \mathrm{NMR}\left(150.91 \mathrm{MHz}, \mathrm{CD}_{2} \mathrm{Cl}_{2}, 213 \mathrm{~K}\right): \delta 163.9(\mathrm{C}, \mathrm{OCN}, 12), 165.4(\mathrm{C}, \mathrm{OCN}, \mathbf{L 2})$, 166.9 (C, OCN, L2'), 144.4 (C, $\left.\mathrm{C}^{2,6} \mathrm{C}_{5} \mathrm{H}_{3} \mathrm{~N}, 12\right), 144.2$ (C, $\left.\mathrm{C}^{2,6} \mathrm{C}_{5} \mathrm{H}_{3} \mathrm{~N}, \mathbf{L 2}\right), 144.0$ (C, $\left.\mathrm{C}^{2,6} \mathrm{C}_{5} \mathrm{H}_{3} \mathrm{~N}, \mathbf{L 2}{ }^{\prime}\right), 140.8\left(\mathrm{CH}, \mathrm{C}^{4} \mathrm{C}_{5} \mathrm{H}_{3} \mathrm{~N}, \mathbf{1 2}\right), 141.7\left(\mathrm{CH}, \mathrm{C}^{4} \mathrm{C}_{5} \mathrm{H}_{3} \mathrm{~N}, \mathbf{L 2}\right), 141.4\left(\mathrm{CH}, \mathrm{C}^{4}\right.$ $\mathrm{C}_{5} \mathrm{H}_{3} \mathrm{~N}, \mathbf{L 2}$ '), 139.9, 138.7 (2 $\left.\mathrm{C}_{\text {arom }}, \mathbf{1 2}\right), 139.1,137.9$ (2 $\left.\mathrm{C}_{\text {arom }}, \mathbf{L 2}\right), 140.2,138.9$ (2 $\left.\mathrm{C}_{\text {arom, L2' }}\right), 129.6\left(\mathrm{CH}_{\text {arom }}, \mathbf{1 2}\right), 129.0$ ( $\left.\mathrm{CH}_{\text {arom }}, \mathbf{L 2}\right), 129.7\left(\mathrm{CH}_{\text {arom }}, \mathbf{L 2}\right.$ '), 128.5 (CH, $\left.\mathrm{C}^{3,5} \mathrm{C}_{5} \mathrm{H}_{3} \mathrm{~N}, \mathbf{1 2}\right), 128.7\left(\mathrm{CH}, \mathrm{C}^{3,5} \mathrm{C}_{5} \mathrm{H}_{3} \mathrm{~N}, 12\right), 128.9\left(\mathrm{CH}, \mathrm{C}^{3,5} \mathrm{C}_{5} \mathrm{H}_{3} \mathrm{~N}, \mathbf{L 2}{ }^{\prime}\right), 127.8$

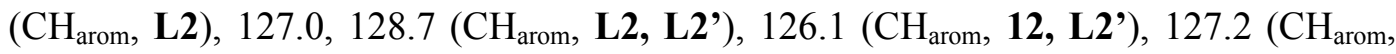
L2), 124.9 ( $\left.\mathrm{CH}_{\text {arom }}, \mathbf{1 2}\right), 123.3$ ( $\left.\mathrm{CH}_{\text {arom }}, \mathbf{L 2}\right), 124.5$ ( $\mathrm{CH}_{\text {arom }}, \mathbf{L 2}$ '), 86.5 ( $\left.\mathrm{CH}, \mathrm{OCH}, \mathbf{1 2}\right)$, 85.5 (CH, OCH, L2), 88.9 (CH, OCH, L2'), 75.9 (CH, NCH, 12), 75.3 (CH, NCH, L2), 75.0 (CH, NCH, L2'), $39.7\left(\mathrm{CH}_{2}, \mathrm{CH}_{2} \mathrm{C}_{\text {arom }}, \mathbf{1 2}\right), 38.6\left(\mathrm{CH}_{2}, \mathrm{CH}_{2} \mathrm{C}_{\text {arom }}, \mathbf{L 2}\right), 39.2\left(\mathrm{CH}_{2}\right.$, $\mathrm{CH}_{2} \mathrm{C}_{\text {arom, }}$ (L2'). 

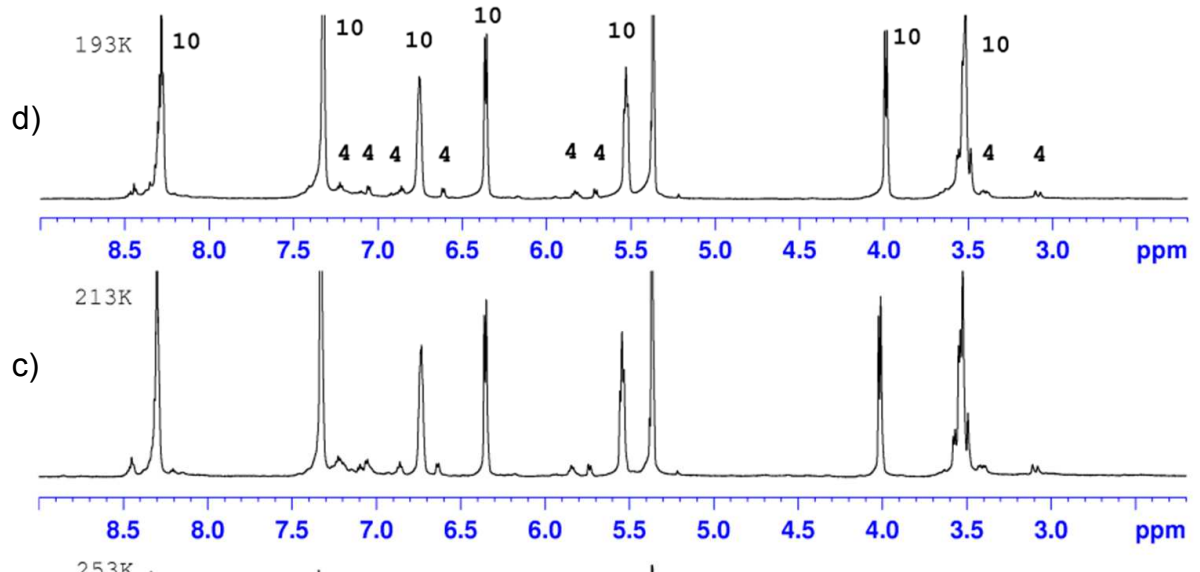

b)
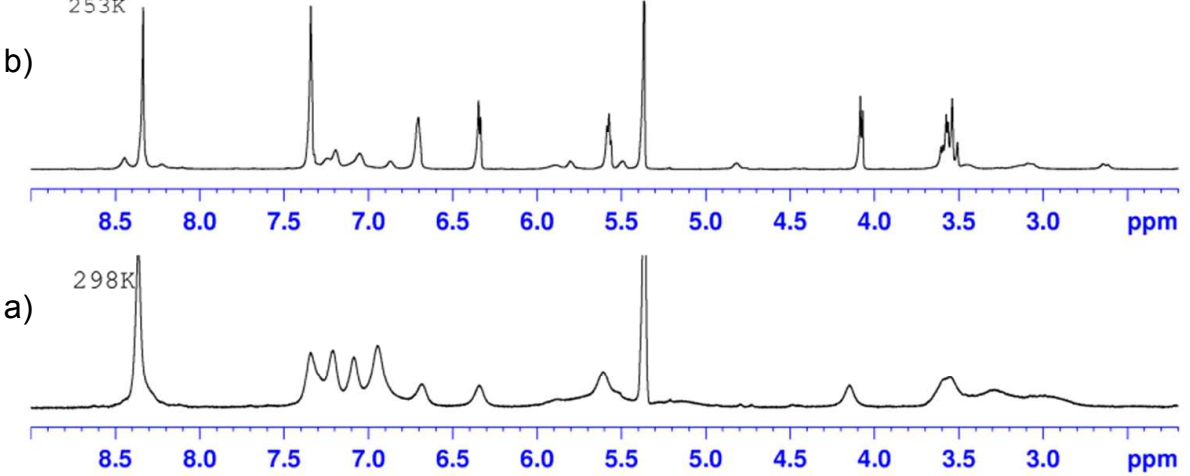

Figure S4. ${ }^{1} \mathrm{H}$ NMR of complex 10 in $\mathrm{CD}_{2} \mathrm{Cl}_{2}$ at (a) $298 \mathrm{~K}$, (b) $253 \mathrm{~K}$, (c) $213 \mathrm{~K}$ and (d) 193 K 

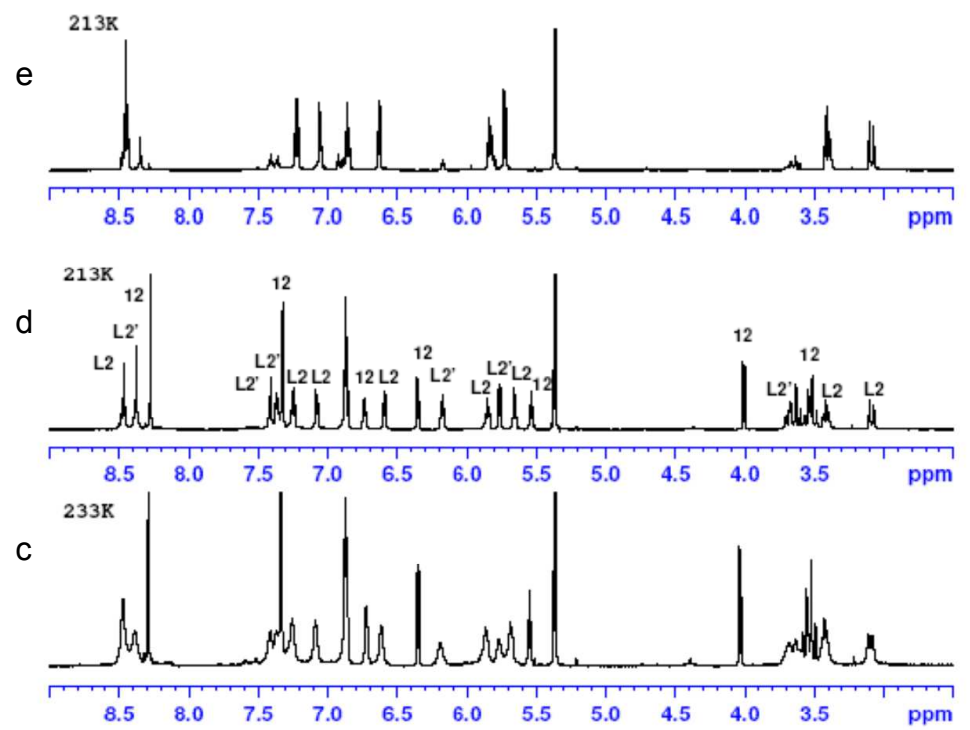

$\mathrm{b}$

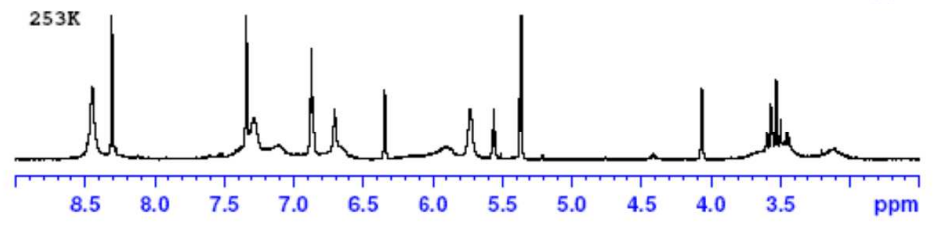

a

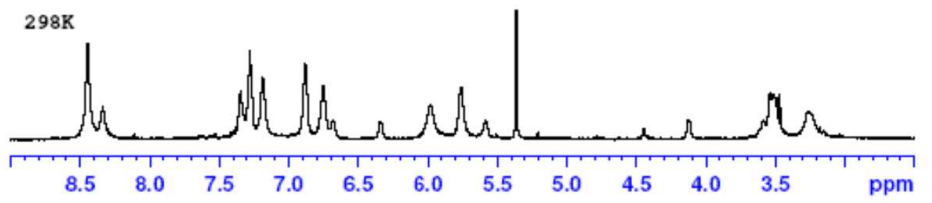

Figure S5. (a) ${ }^{1} \mathrm{H} N M R$ of complex 12 in $\mathrm{CD}_{2} \mathrm{Cl}_{2}$ at $298 \mathrm{~K}$, (b) $253 \mathrm{~K}$, (c) $233 \mathrm{~K}$ and (d) $213 \mathrm{~K}$; $\left.\mathbf{L 2}, \mathbf{L 2}^{\prime}=\left[\mathrm{Ag}_{2}(\text { indane-pybox })_{2}\right]\left[\mathrm{PF}_{6}\right]_{2} ; \mathbf{1 2}=\left[\mathrm{Ag}_{2} \text { (indane-pybox }\right)_{3}\right]\left[\mathrm{PF}_{6}\right]_{2}$. (e) ${ }^{1} \mathrm{H} N M R$ of complex 4 in $\mathrm{CD}_{2} \mathrm{Cl}_{2}$ at $213 \mathrm{~K}$.

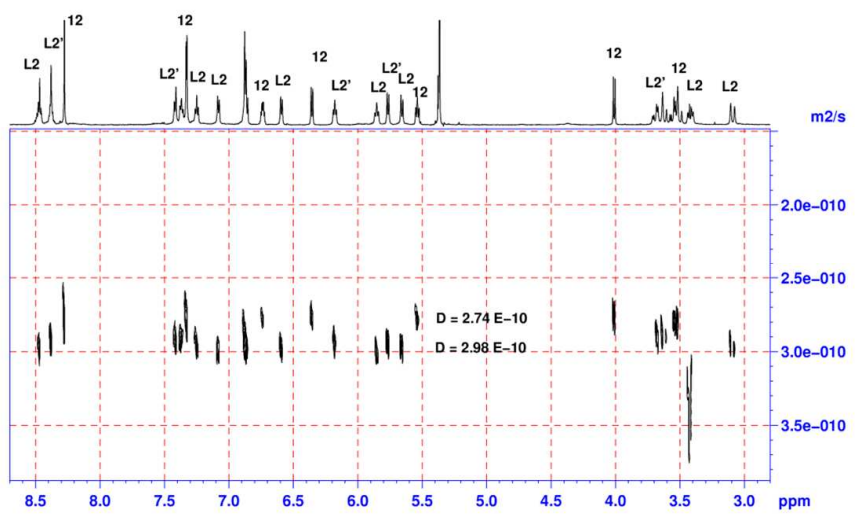

Figure $56 .{ }^{1} \mathrm{H}$ DOSY spectrum of complex 12 at $213 \mathrm{~K}$. 
NMR spectra for complexes 1,4 ,

\section{Complex 1.}

1H RMN AV 400
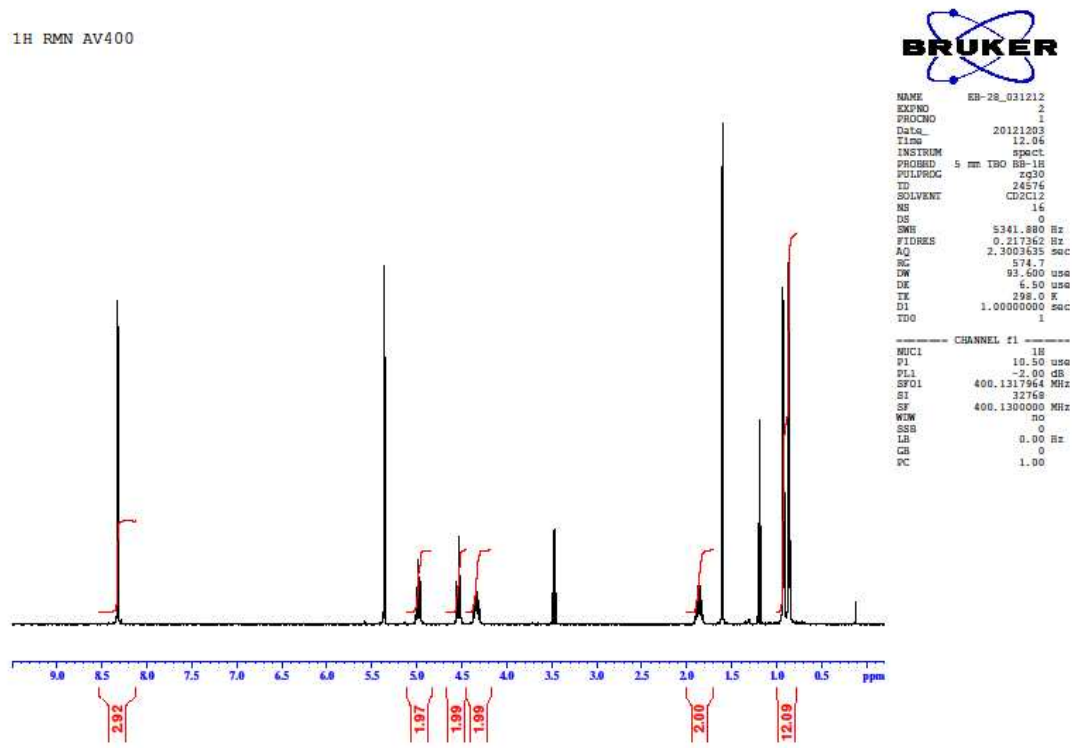

Complex 1. ${ }^{1} \mathrm{H}$ RMN $298 \mathrm{~K}\left(\mathrm{CD}_{2} \mathrm{Cl}_{2}\right)$
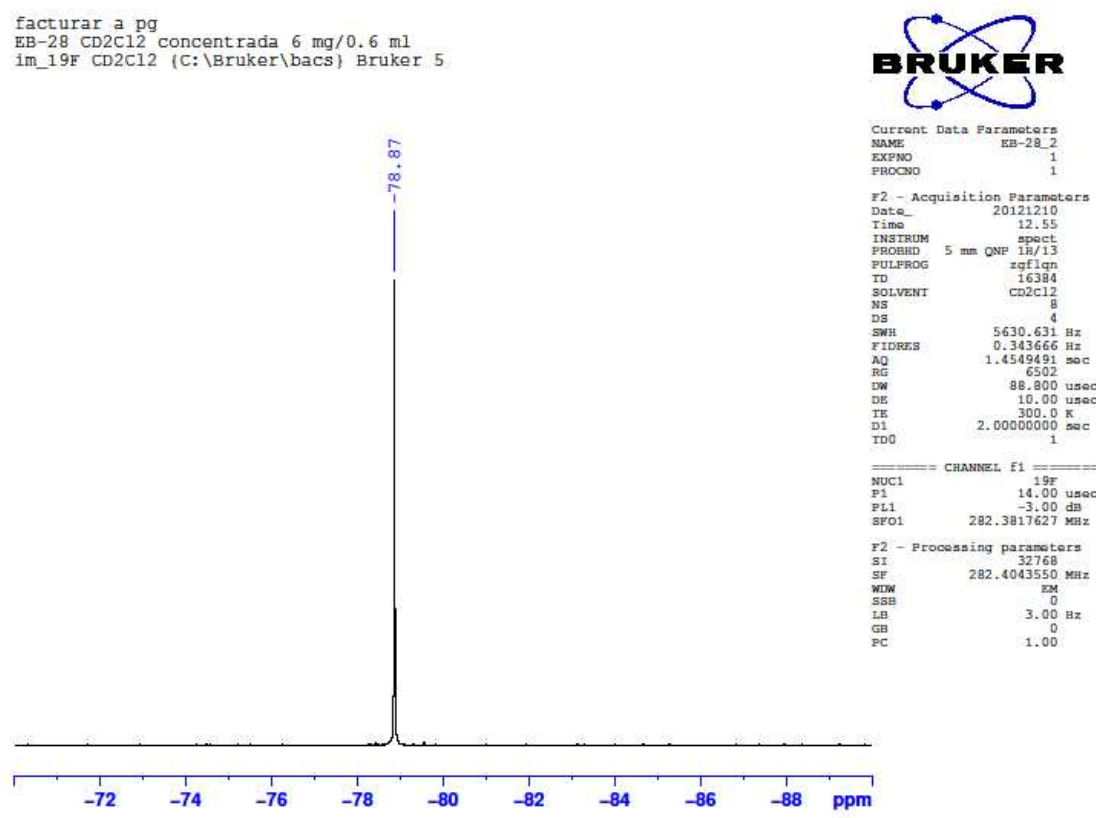

Complex 1. ${ }^{19} \mathrm{~F}$ RMN 298K $\left(\mathrm{CD}_{2} \mathrm{Cl}_{2}\right)$ 

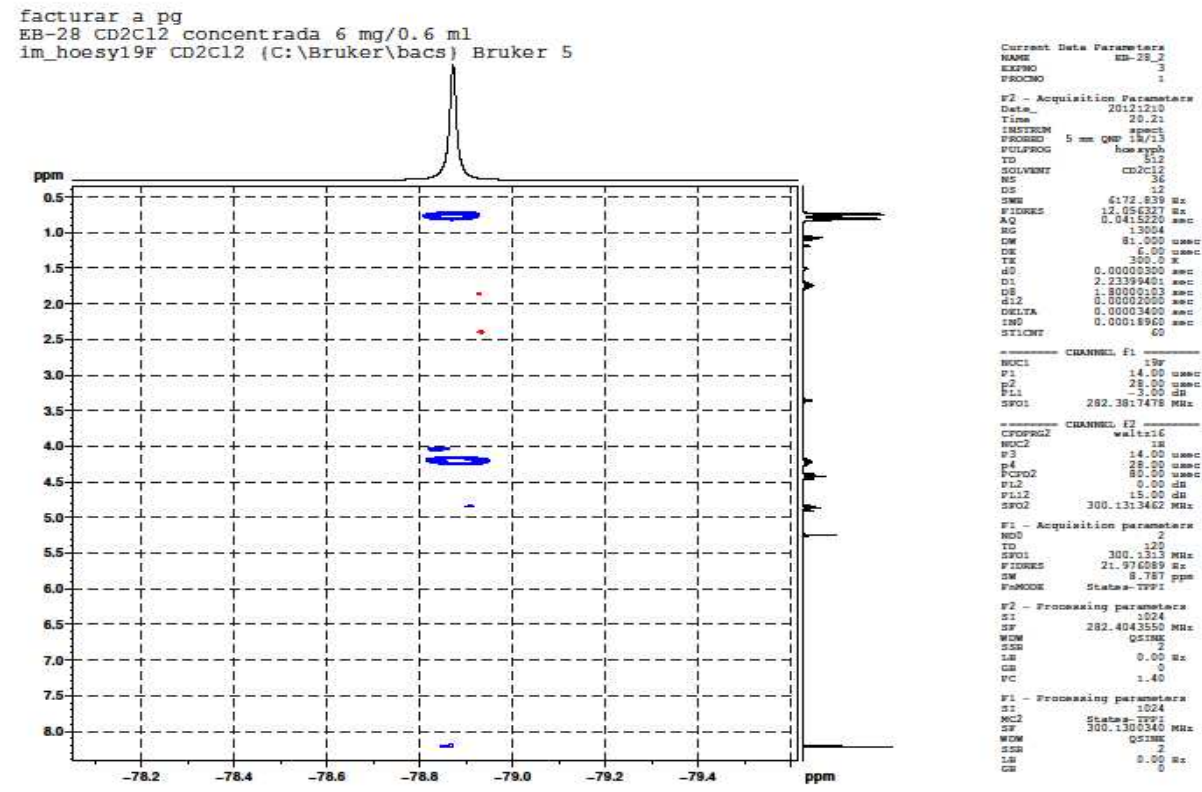

Complex 1. ${ }^{1} \mathrm{H}{ }^{19} \mathrm{~F}$ HOESY $298 \mathrm{~K}\left(\mathrm{CD}_{2} \mathrm{Cl}_{2}\right)$

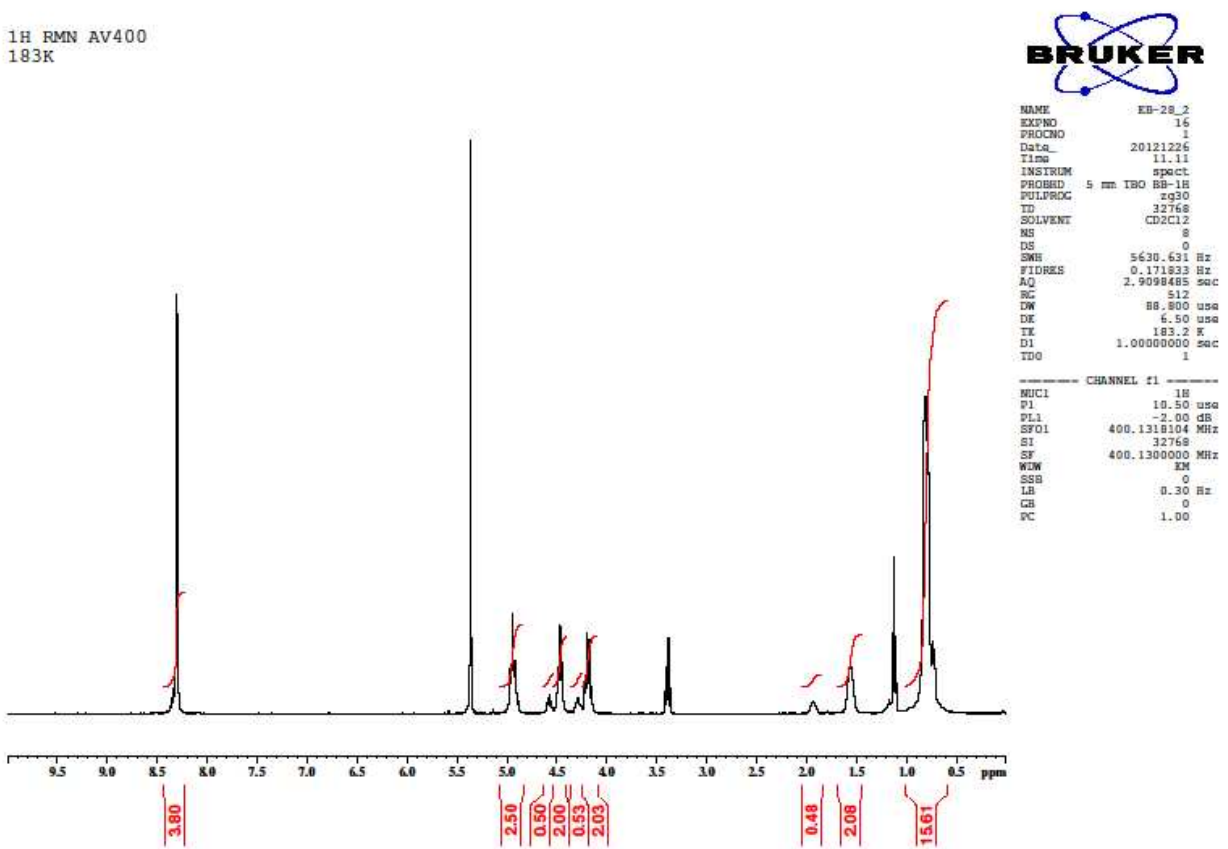

Complex 1. ${ }^{1} \mathrm{H}$ RMN $183 \mathrm{~K}\left(\mathrm{CD}_{2} \mathrm{Cl}_{2}\right)$ 

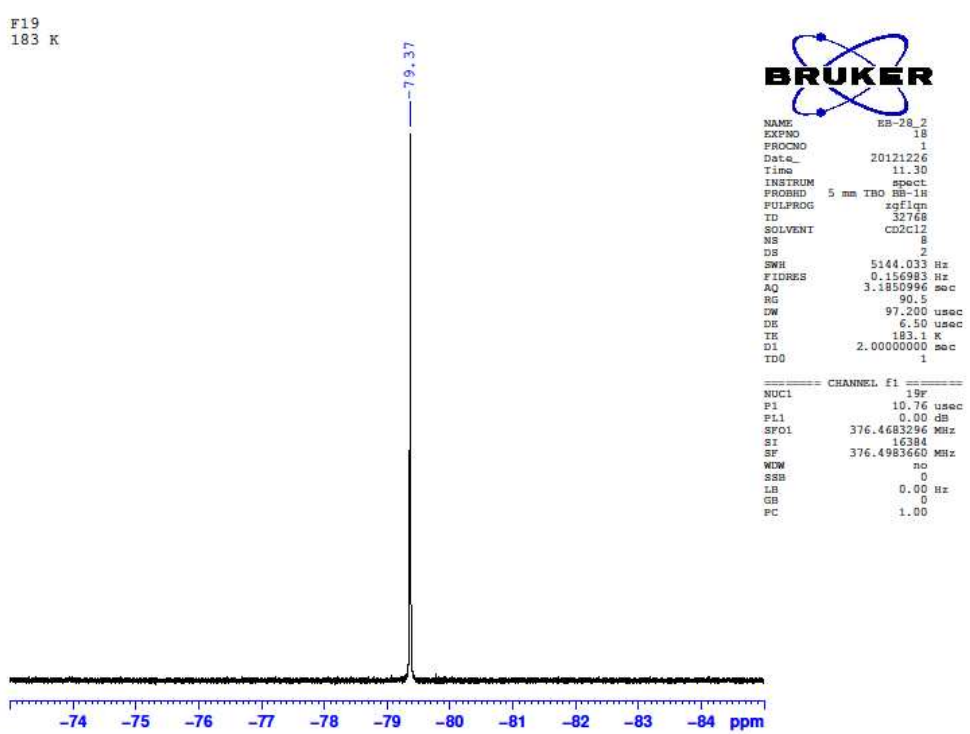

Complex 1. ${ }^{19} \mathrm{~F}$ RMN $183 \mathrm{~K}\left(\mathrm{CD}_{2} \mathrm{Cl}_{2}\right)$

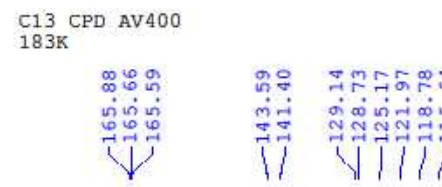

.



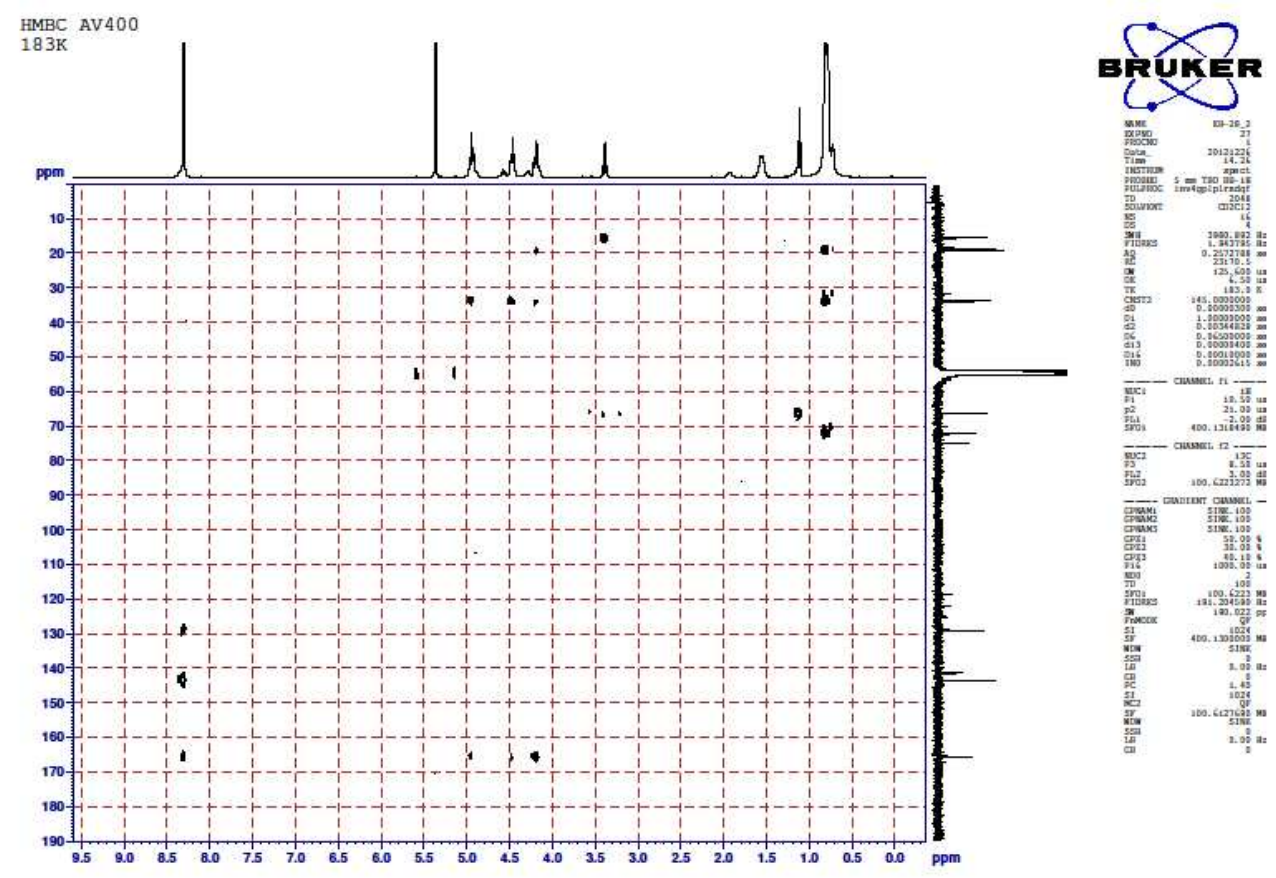

Complex 1. ${ }^{1} \mathrm{H}^{13} \mathrm{C}$ HMB $183 \mathrm{~K}\left(\mathrm{CD}_{2} \mathrm{Cl}_{2}\right)$
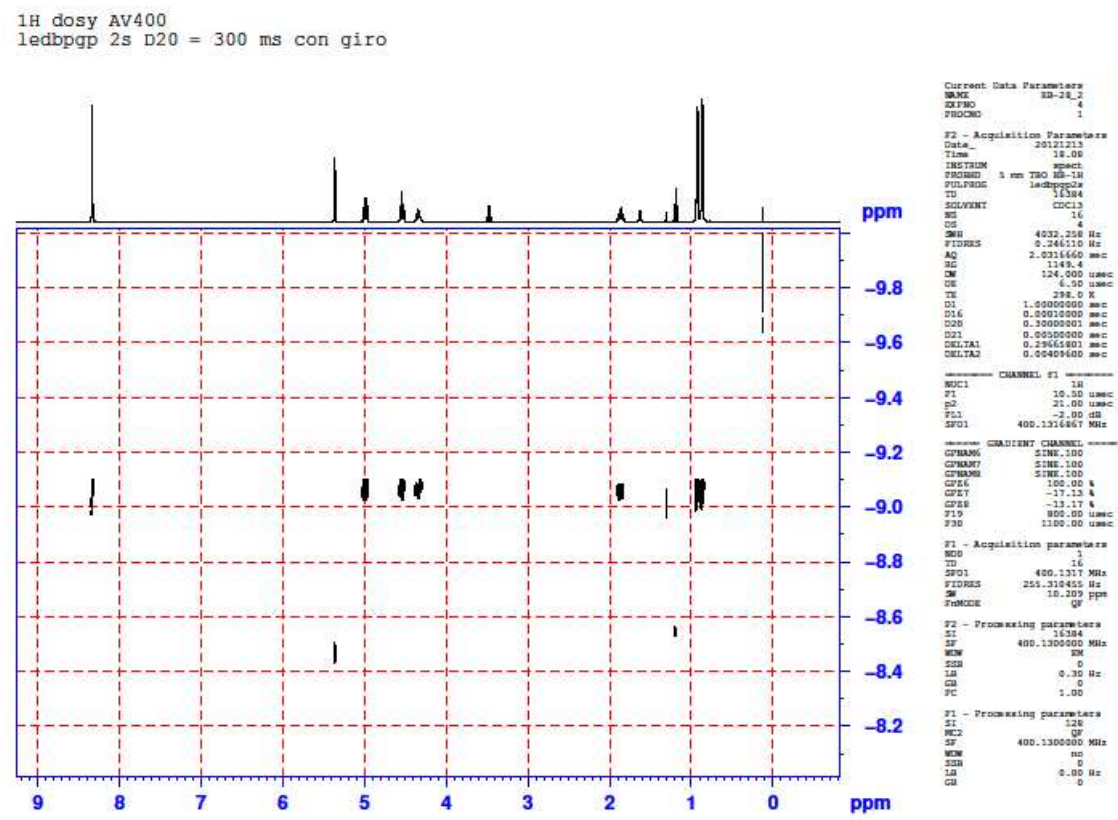

Complex 1. ${ }^{1} \mathrm{H}$ DOSY $298 \mathrm{~K}\left(\mathrm{CD}_{2} \mathrm{Cl}_{2}\right)$ 

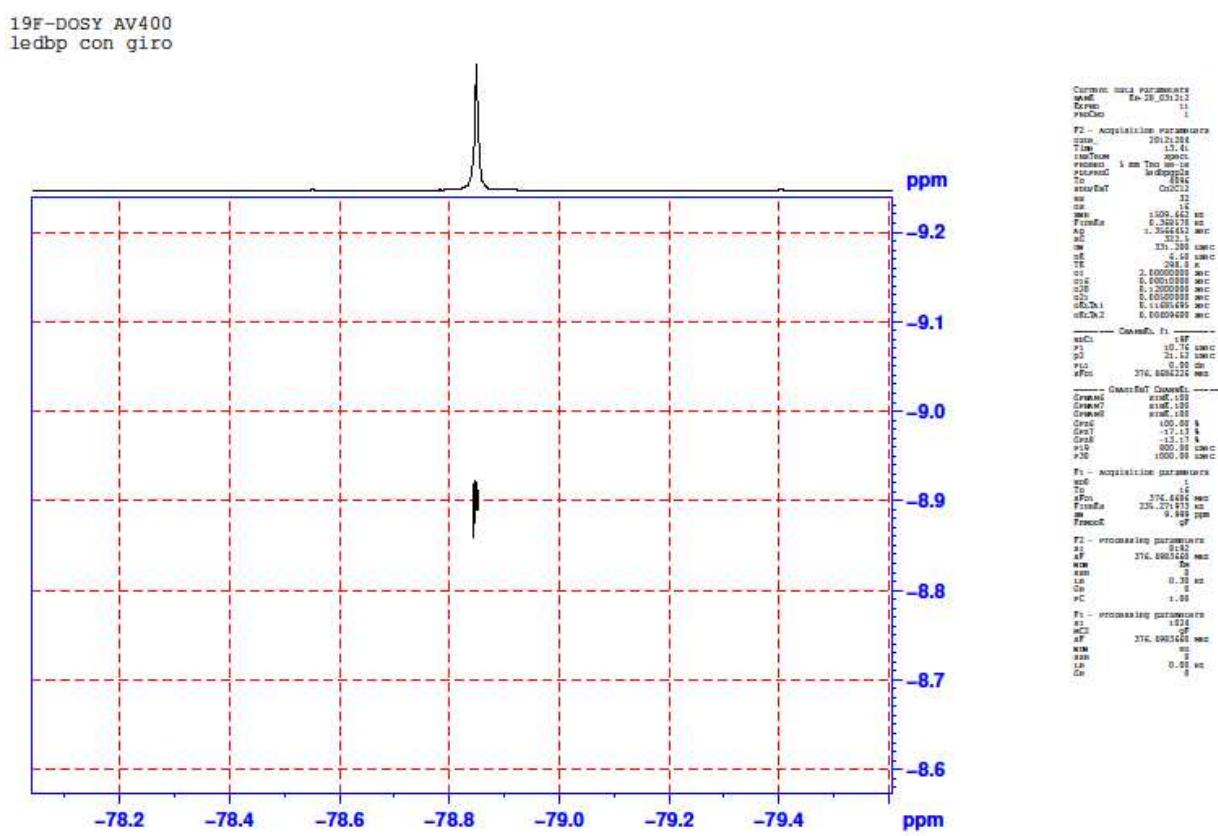

Complex 1. ${ }^{19} \mathrm{~F}$ DOSY $298 \mathrm{~K}\left(\mathrm{CD}_{2} \mathrm{Cl}_{2}\right)$

\section{Complex 6}

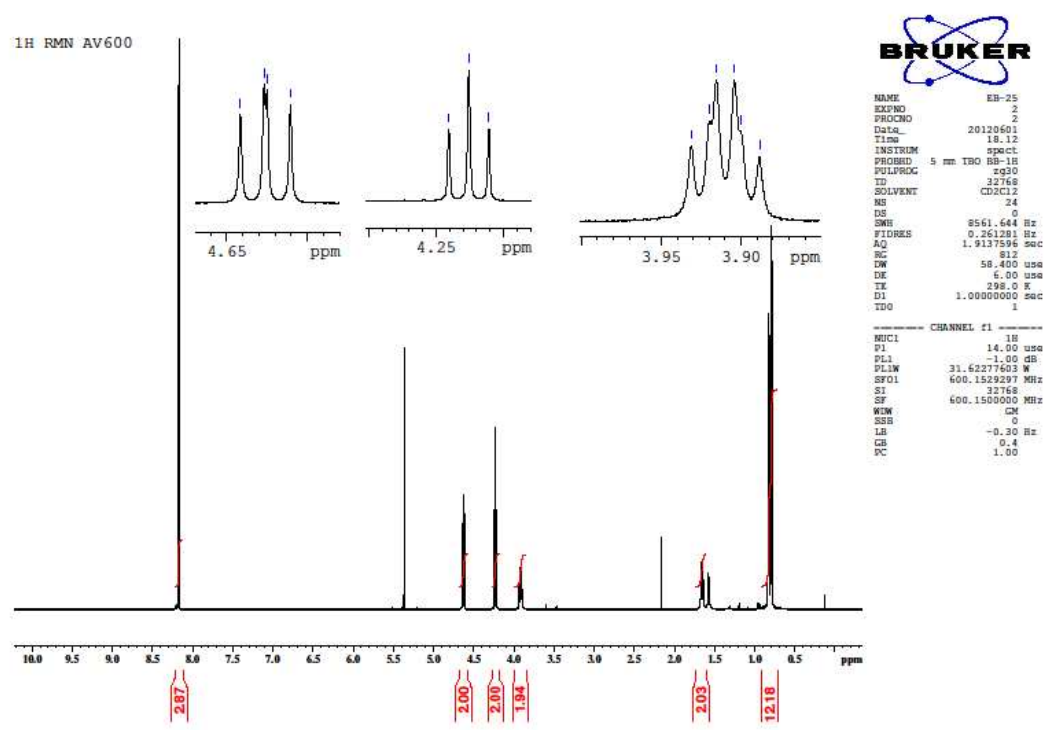

Complex 6. ${ }^{1} \mathrm{H}$ RMN $298 \mathrm{~K}\left(\mathrm{CD}_{2} \mathrm{Cl}_{2}\right)$ 


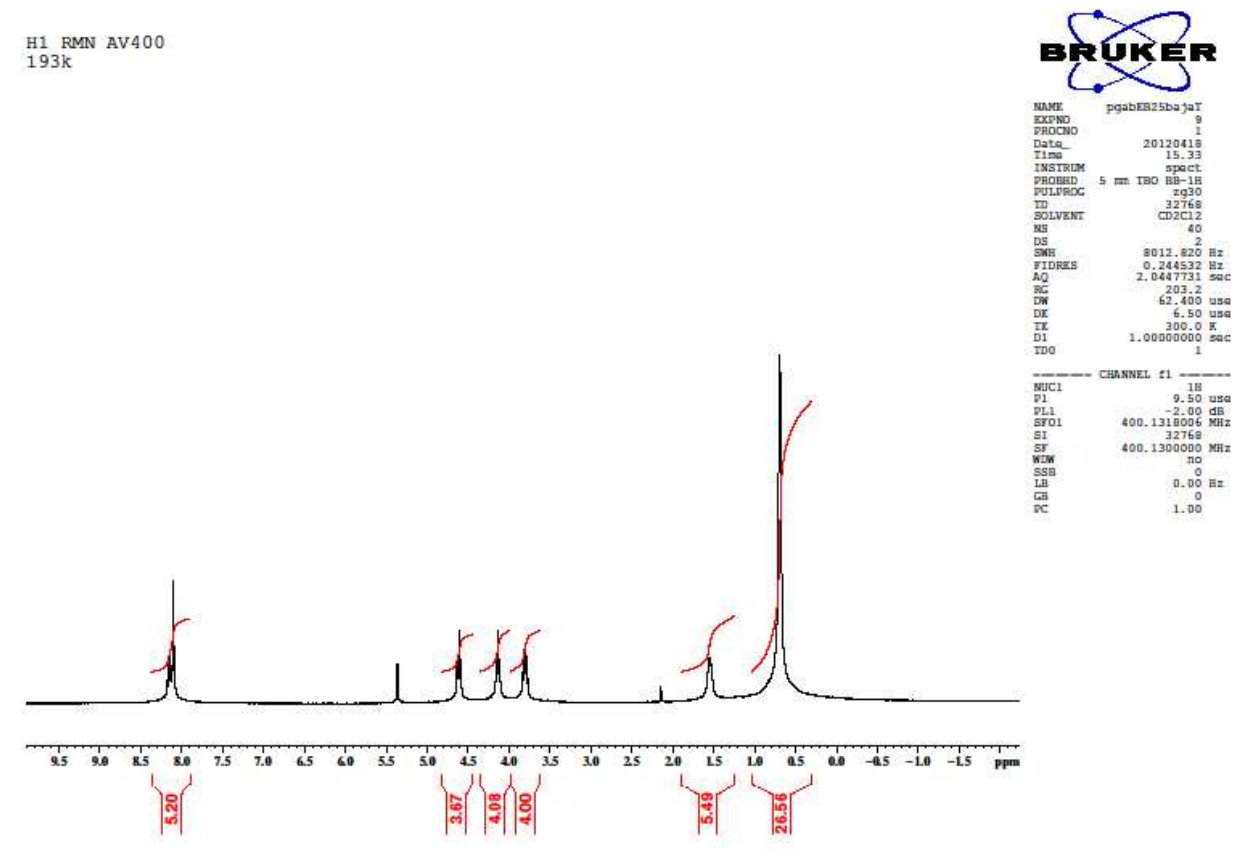

Complex 6. ${ }^{1} \mathrm{H}$ RMN $193 \mathrm{~K}\left(\mathrm{CD}_{2} \mathrm{Cl}_{2}\right)$

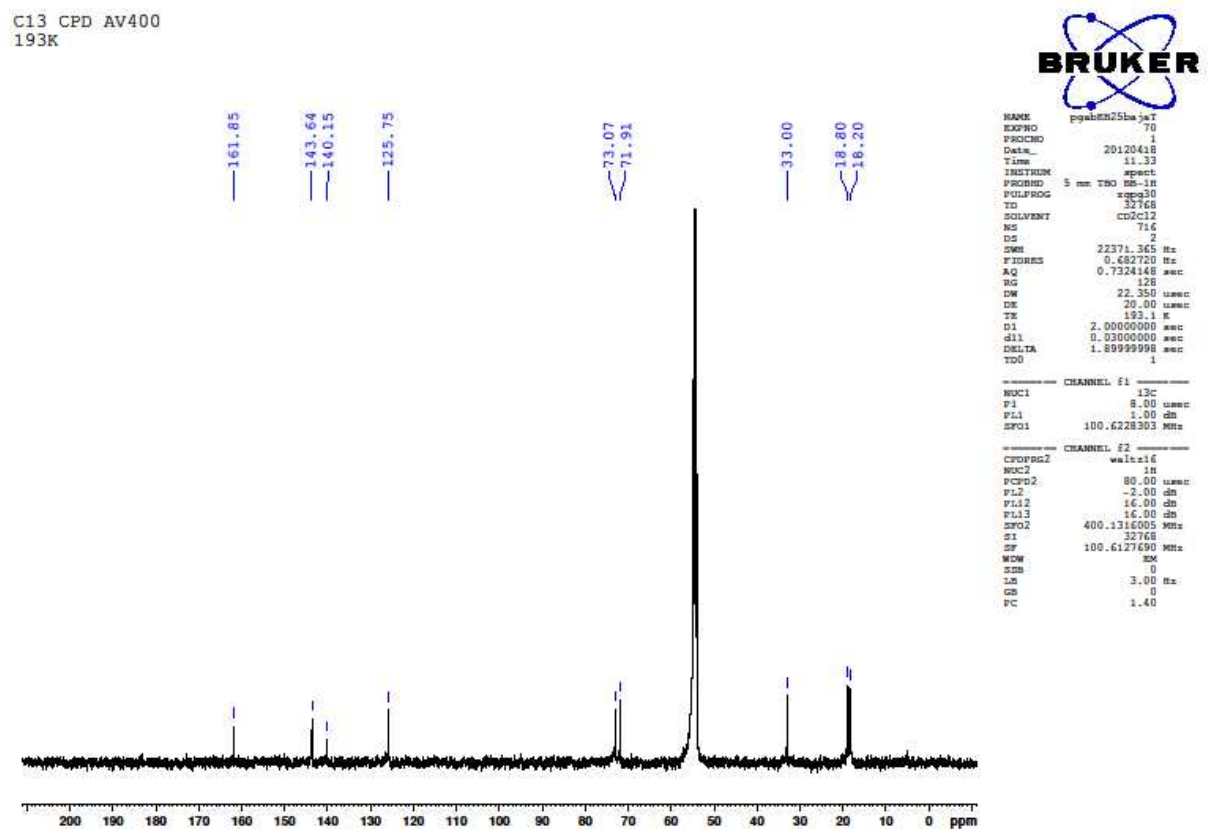

Complex 6. ${ }^{13} \mathrm{C}$ RMN $193 \mathrm{~K}\left(\mathrm{CD}_{2} \mathrm{Cl}_{2}\right)$ 

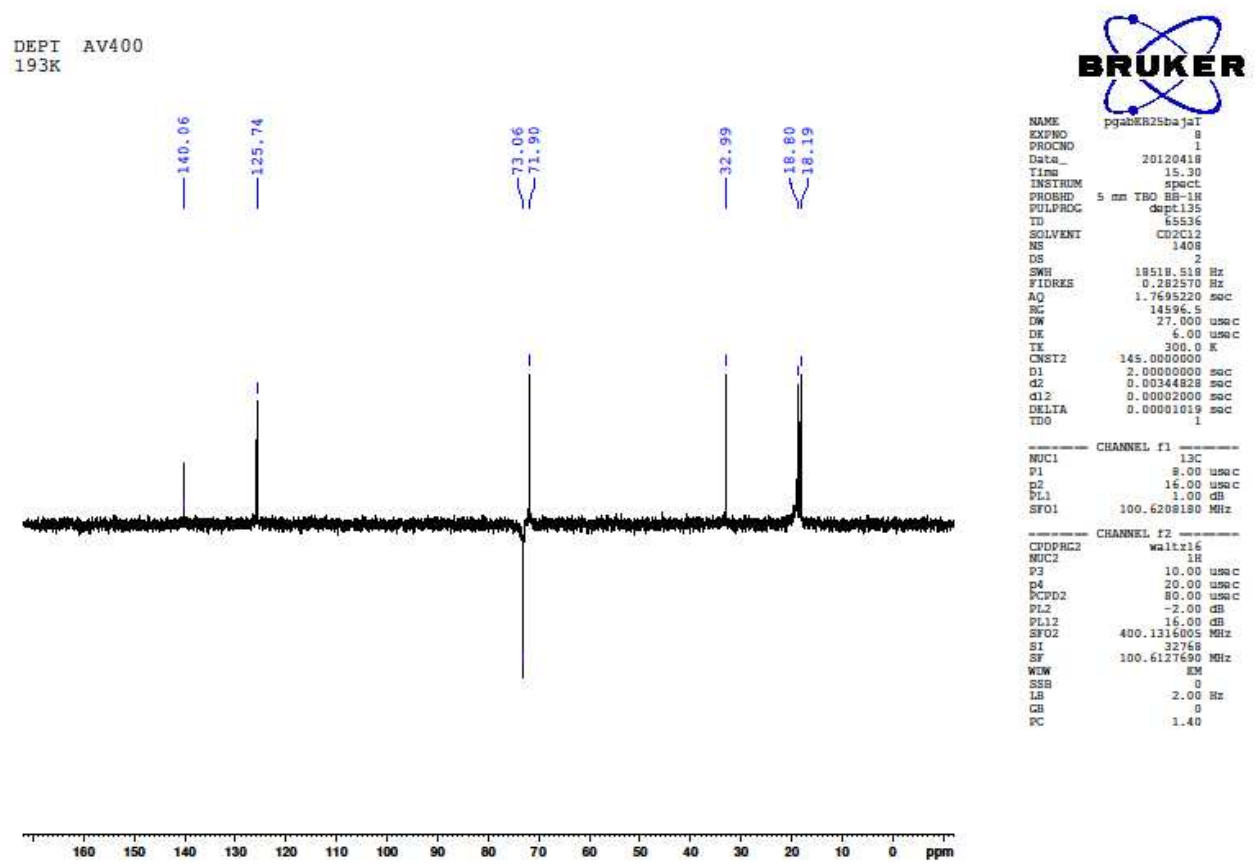

Complex 6. DEPT135 193K $\left(\mathrm{CD}_{2} \mathrm{Cl}_{2}\right)$
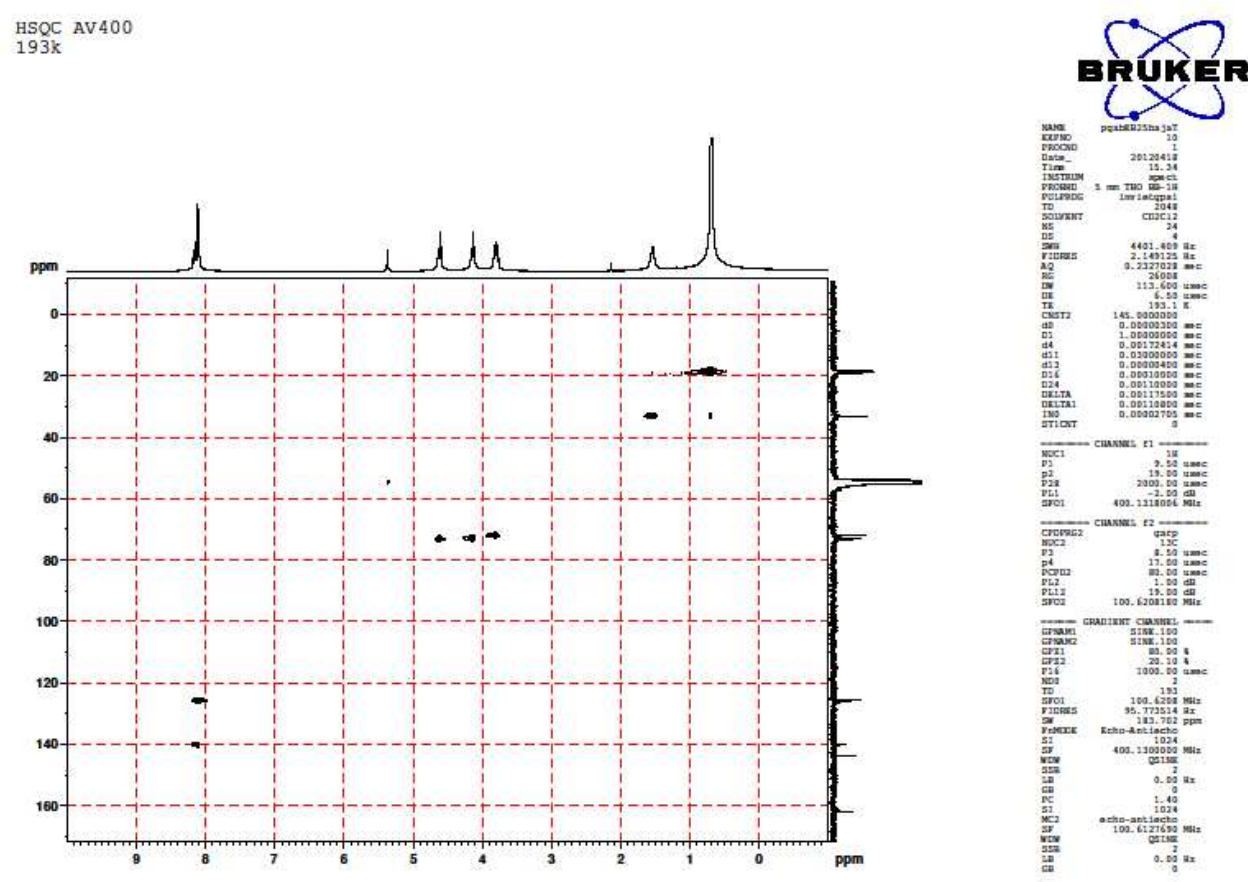

Complex 6. ${ }^{1} \mathrm{H}^{13} \mathrm{C}$ HSQC $193 \mathrm{~K}\left(\mathrm{CD}_{2} \mathrm{Cl}_{2}\right)$ 


\section{Complex 4}

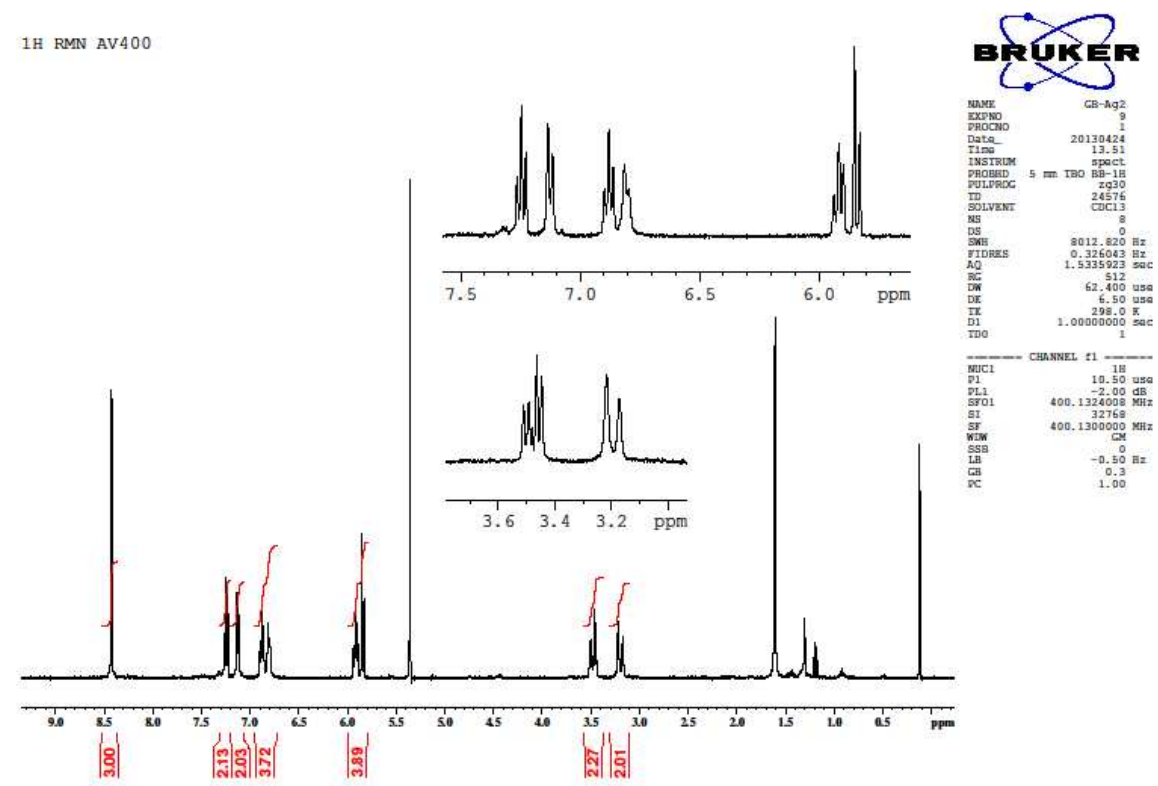

Complex 4. ${ }^{1} \mathrm{H}$ RMN $298 \mathrm{~K}\left(\mathrm{CDCl}_{3}\right)$

C13 CPD AV400

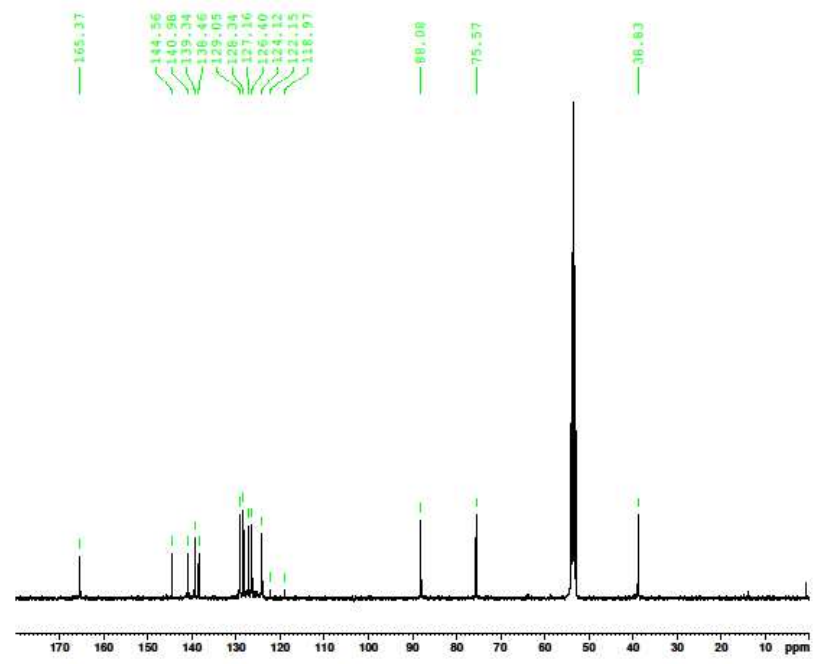

Complex 4. ${ }^{13} \mathrm{C}$ RMN $298 \mathrm{~K}\left(\mathrm{CD}_{2} \mathrm{Cl}_{2}\right)$

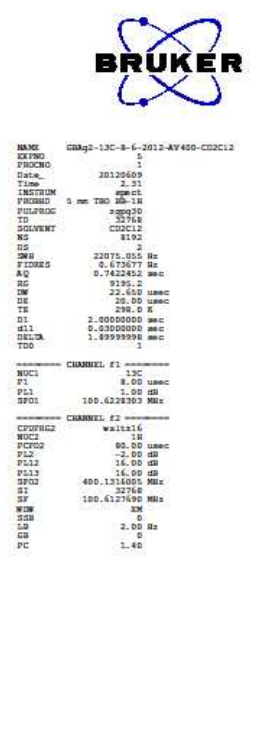




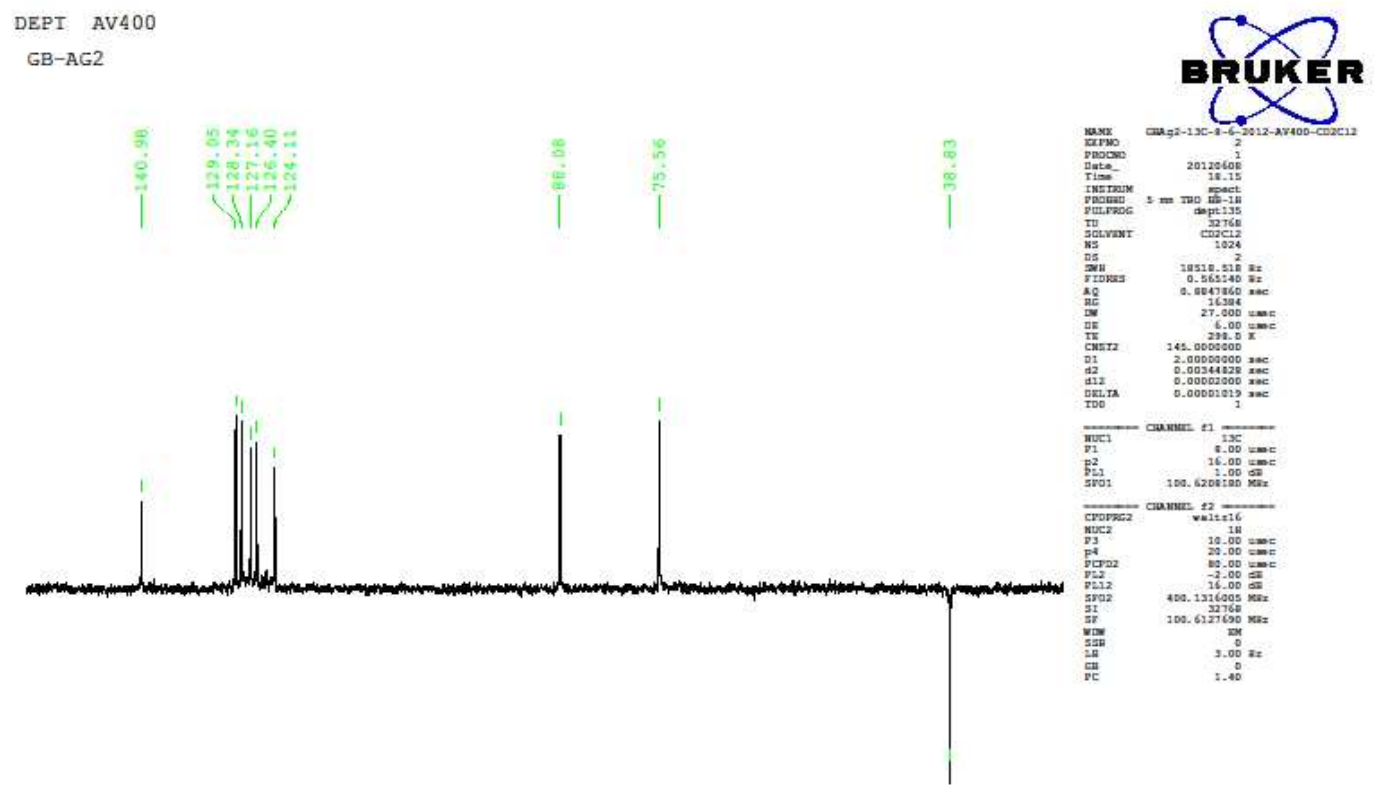

$\begin{array}{llllllllllllllllllllllllllllll}150 & 145 & 140 & 135 & 130 & 125 & 120 & 115 & 110 & 105 & 100 & 95 & 90 & 85 & 80 & 75 & 70 & 65 & 60 & 55 & 50 & 45 & 40 & 35 & \text { ppm }\end{array}$

Complex 4. DEPT135 298K $\left(\mathrm{CD}_{2} \mathrm{Cl}_{2}\right)$

HSQC AV 400

Cat-AC2
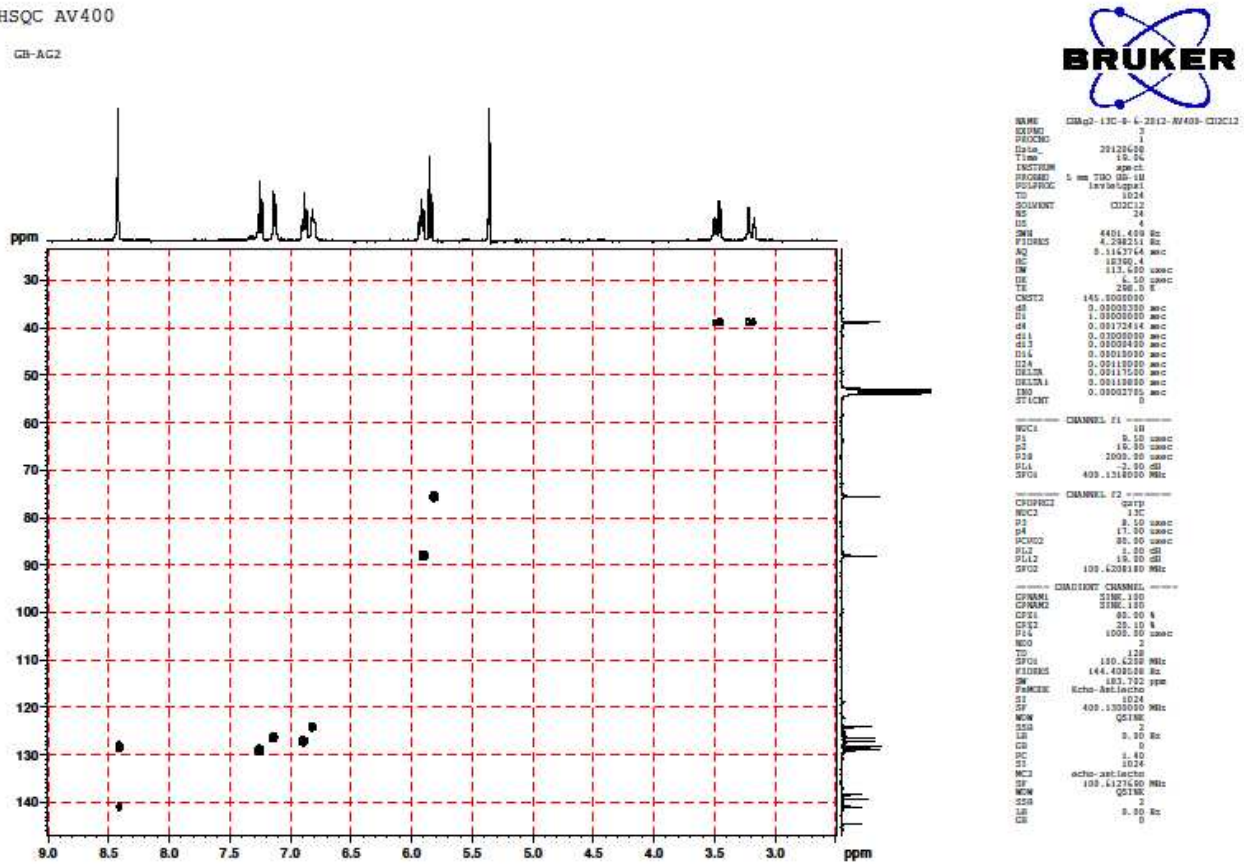

Complex 4. ${ }^{1} \mathrm{H}^{13} \mathrm{C}$ HSQC $298 \mathrm{~K}\left(\mathrm{CD}_{2} \mathrm{Cl}_{2}\right)$ 
cosy CD2C12 \{C:\Bruker\bacs\} Bruker 10

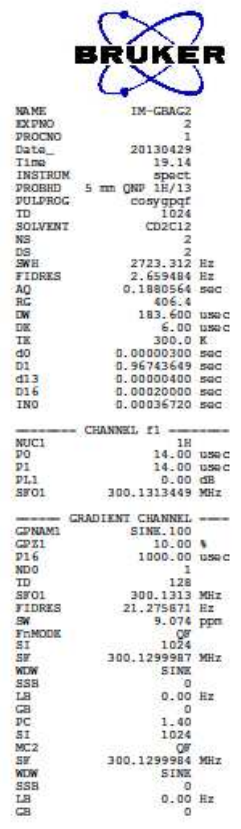

Complex 4. COSY 298K $\left(\mathrm{CD}_{2} \mathrm{Cl}_{2}\right)$
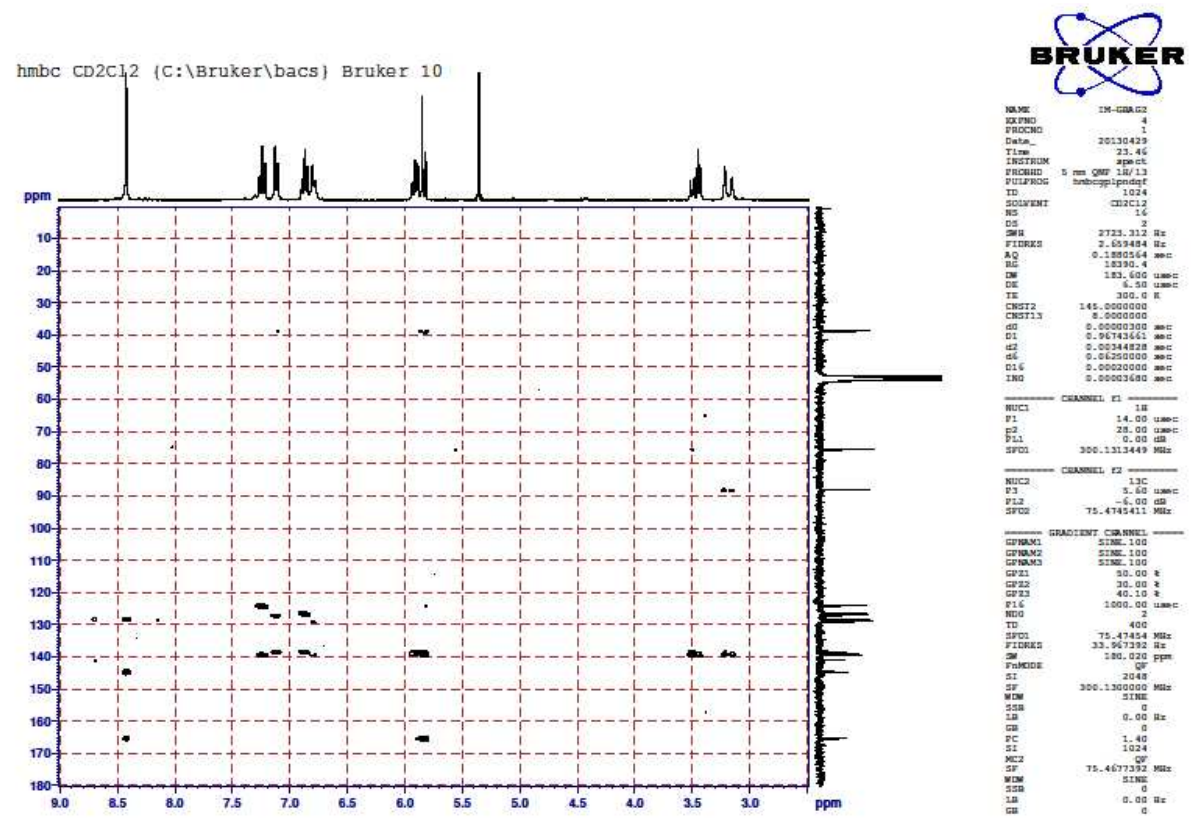

Complex 4. ${ }^{1} \mathrm{H}^{13} \mathrm{C}$ HMBC $298 \mathrm{~K}$ 


\section{हRUKदR \\ c. $\times$}

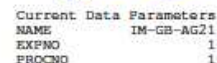

F2- Aequisition parametors
Date
Time
$\begin{aligned} & 20130422 \\ & 13.23\end{aligned}$

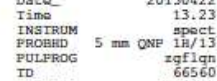

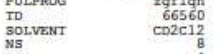

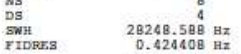

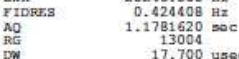

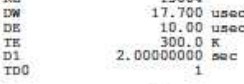

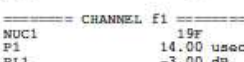

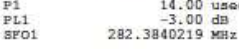

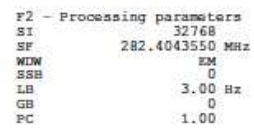

Complex 4. ${ }^{19} \mathrm{~F}$ RMN $298 \mathrm{~K}\left(\mathrm{CD}_{2} \mathrm{Cl}_{2}\right)$
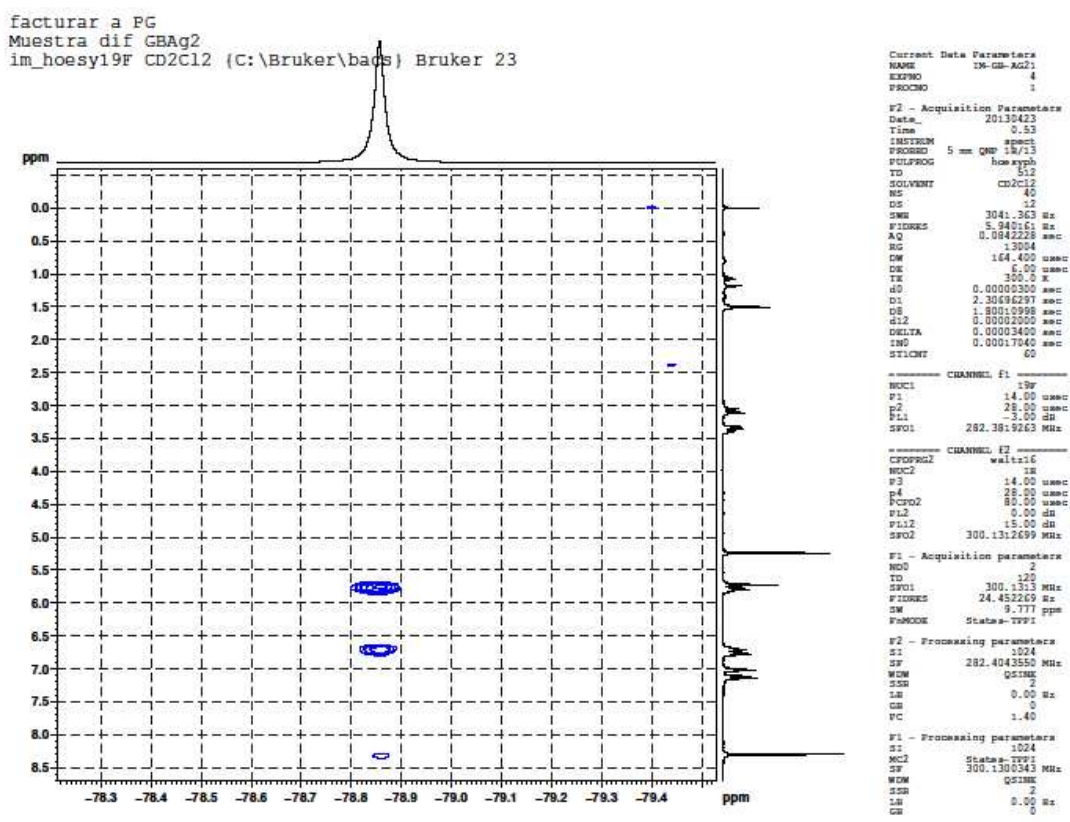

Complex 4. ${ }^{1} \mathrm{H}^{19} \mathrm{~F}$ HOESY $298 \mathrm{~K}\left(\mathrm{CD}_{2} \mathrm{Cl}_{2}\right)$ 

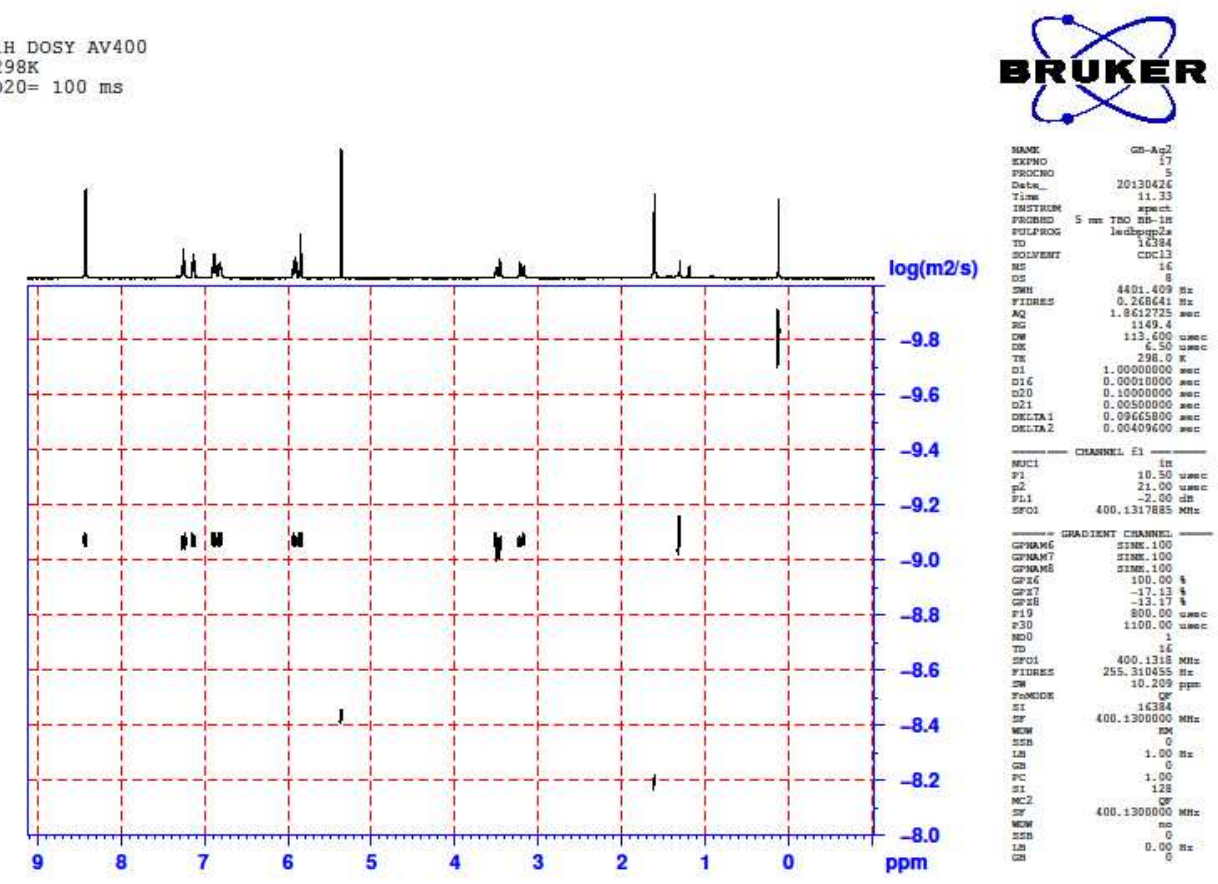

Complex 4. ${ }^{1} \mathrm{H}$ DOSY 298K D20 $=100 \mathrm{~ms}$

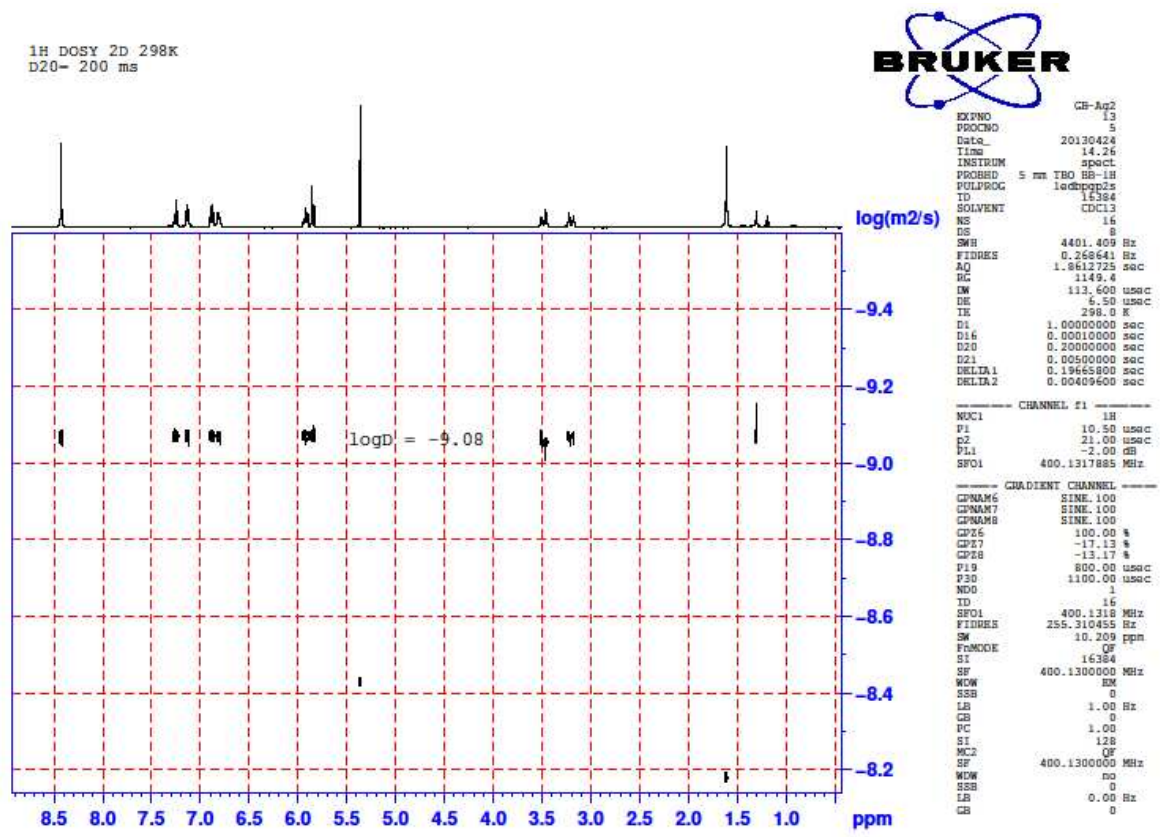

Complex 4. ${ }^{1} \mathrm{H}$ DOSY 298K D20 $=200 \mathrm{~ms}$ 

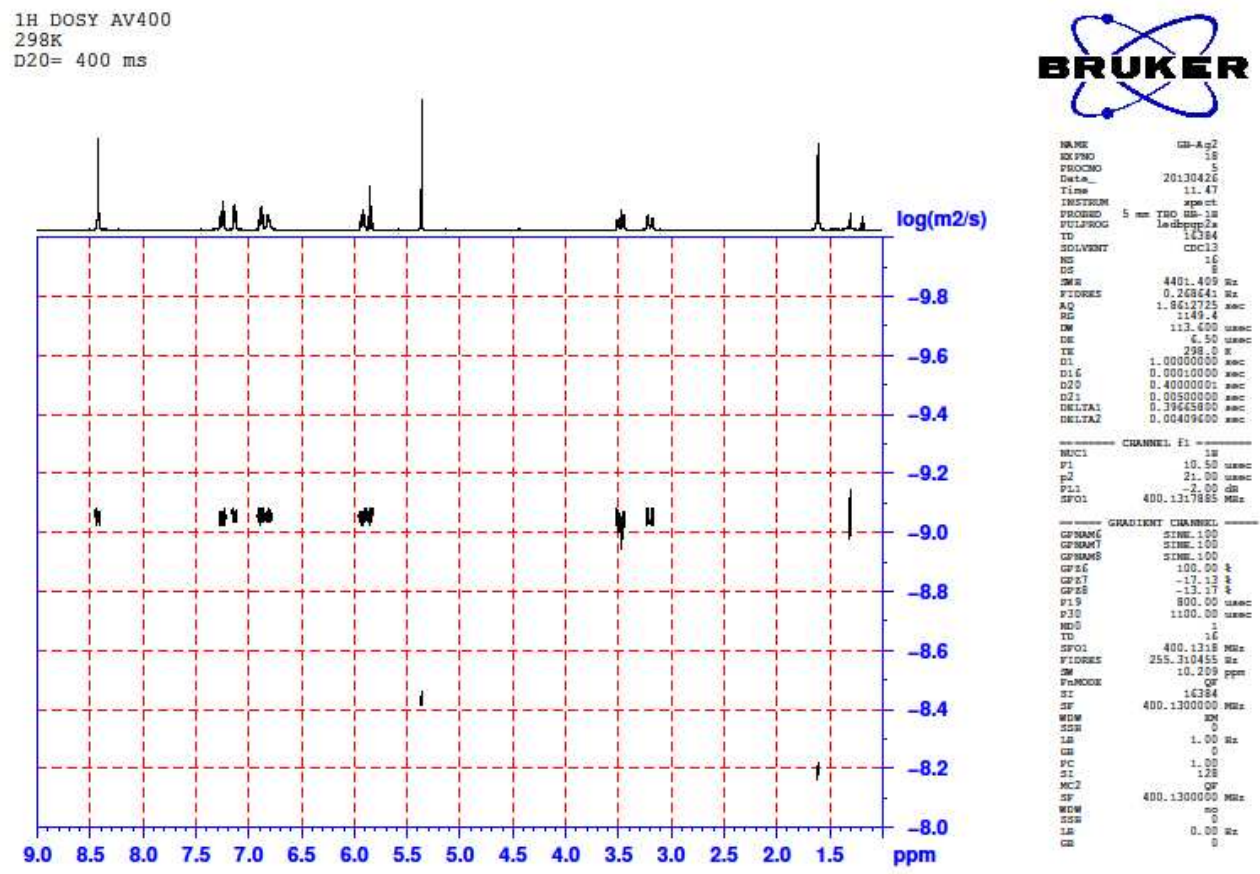

\section{Complex 4. ${ }^{1} \mathrm{H}$ DOSY 298K D20 $=400 \mathrm{~ms}$}

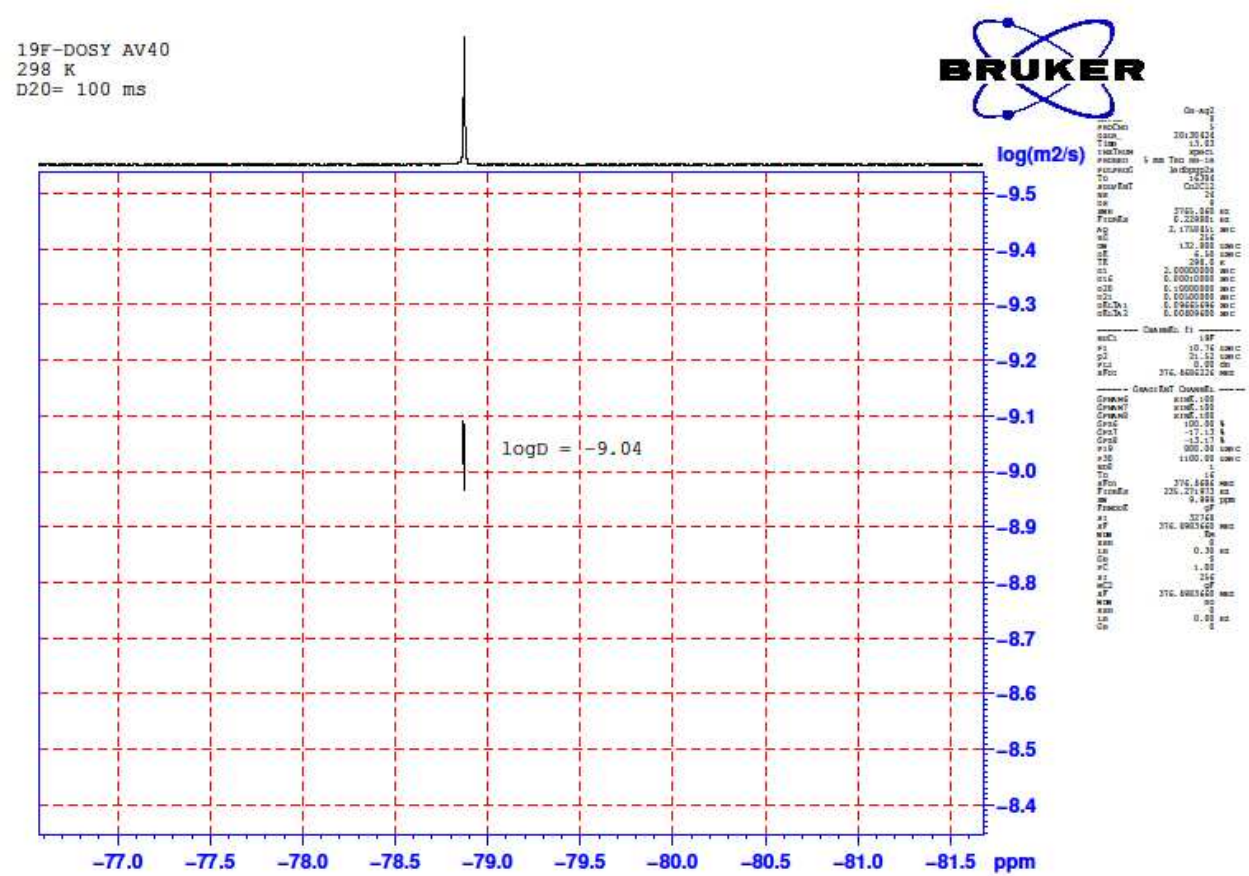

Complex 4. ${ }^{19} \mathrm{~F}$ DOSY 298K D20 $=100 \mathrm{~ms}$ 


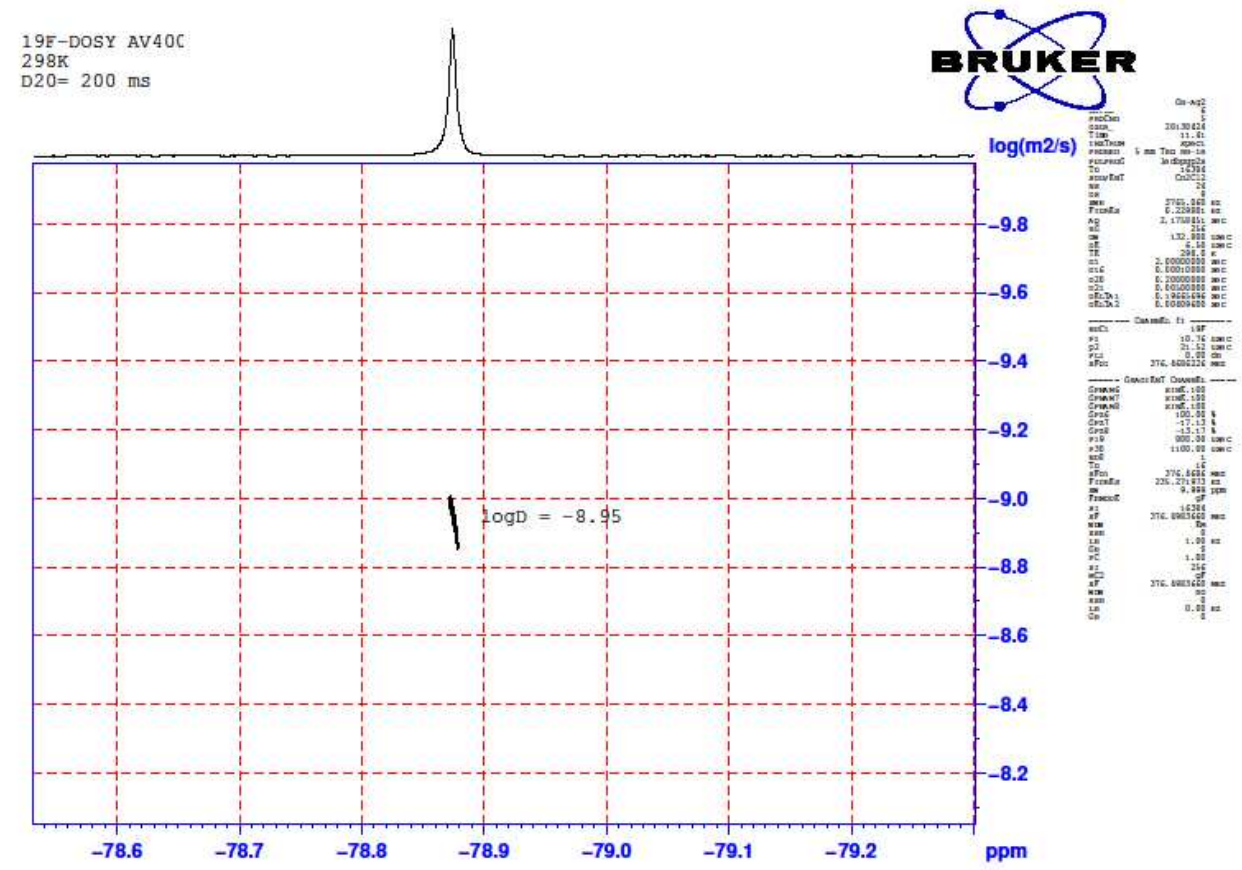

Complex 4. ${ }^{19} \mathrm{~F}$ DOSY 298K D20 $=200 \mathrm{~ms}$
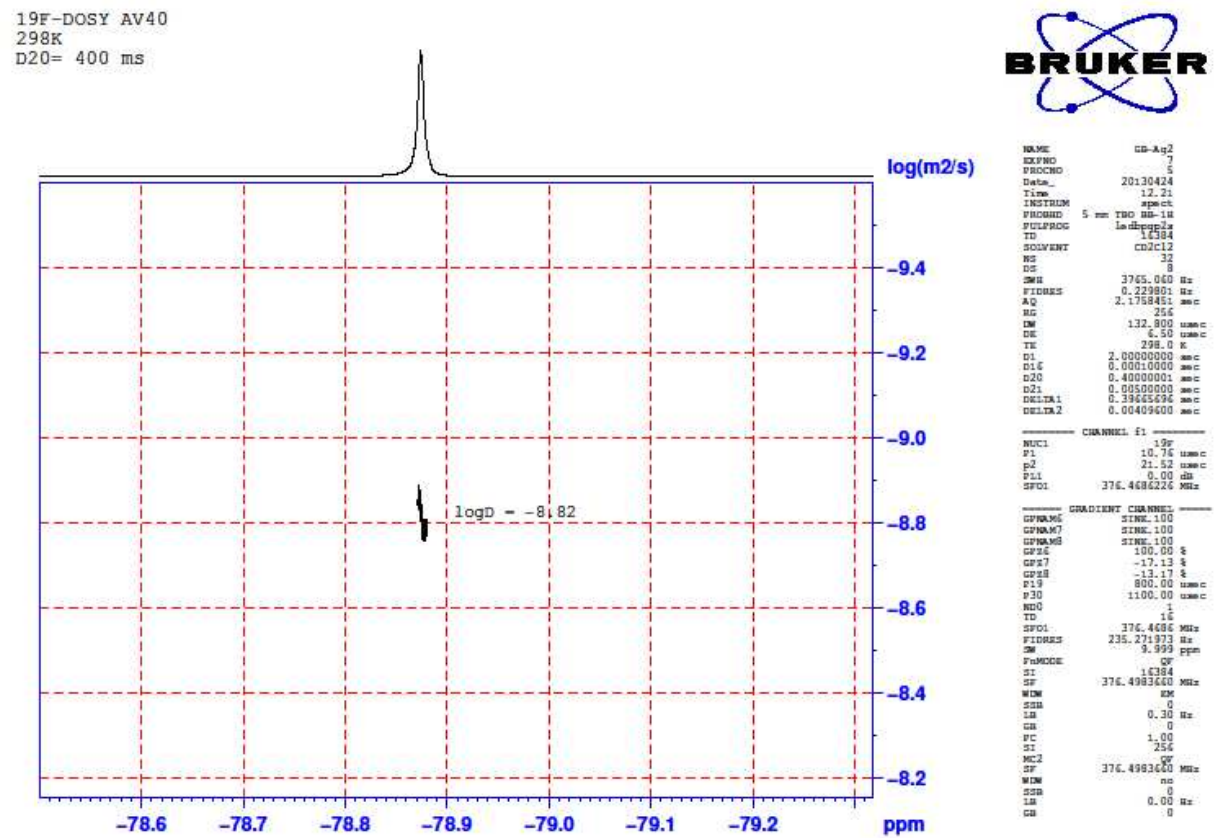

Complex 4. ${ }^{19} \mathrm{~F}$ DOSY 298K D20 $=400 \mathrm{~ms}$ 


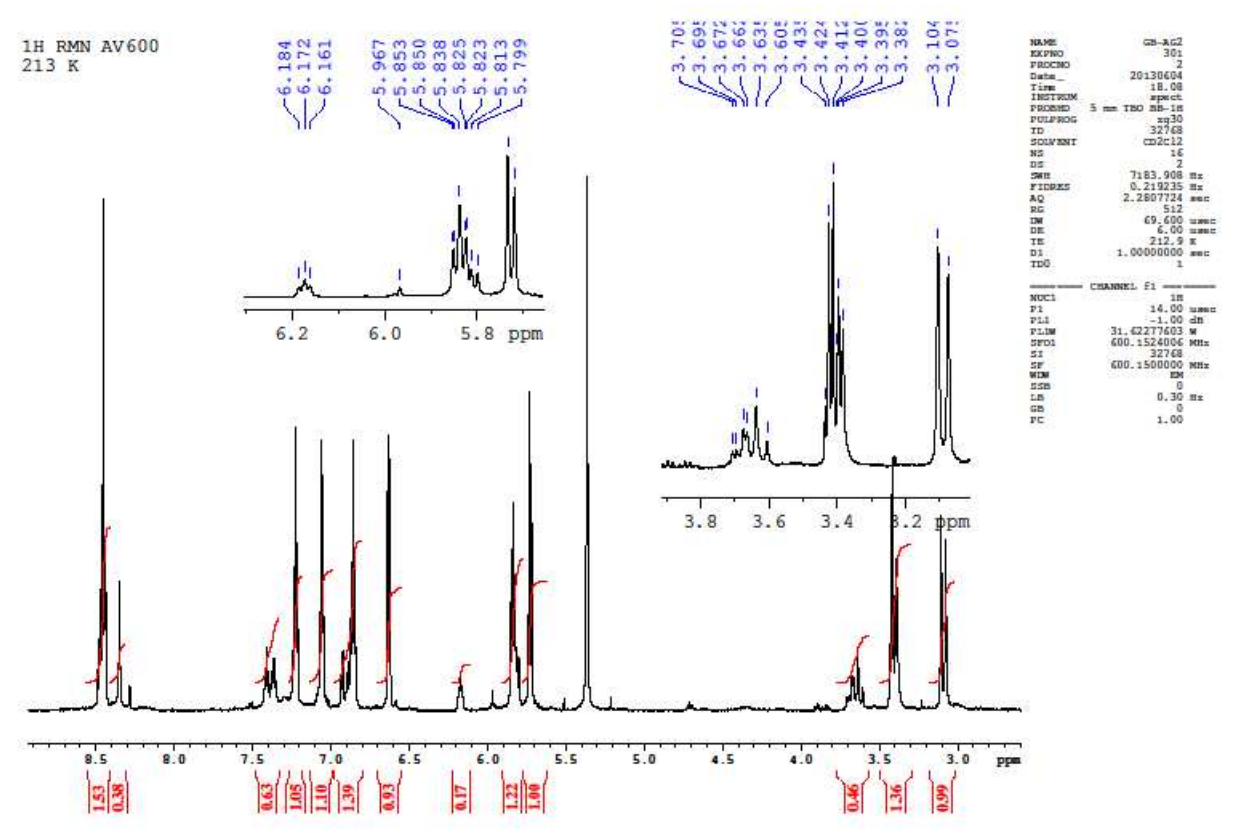

Complex 4. ${ }^{1} \mathrm{H}$ RMN 213K

\section{Complex 10}

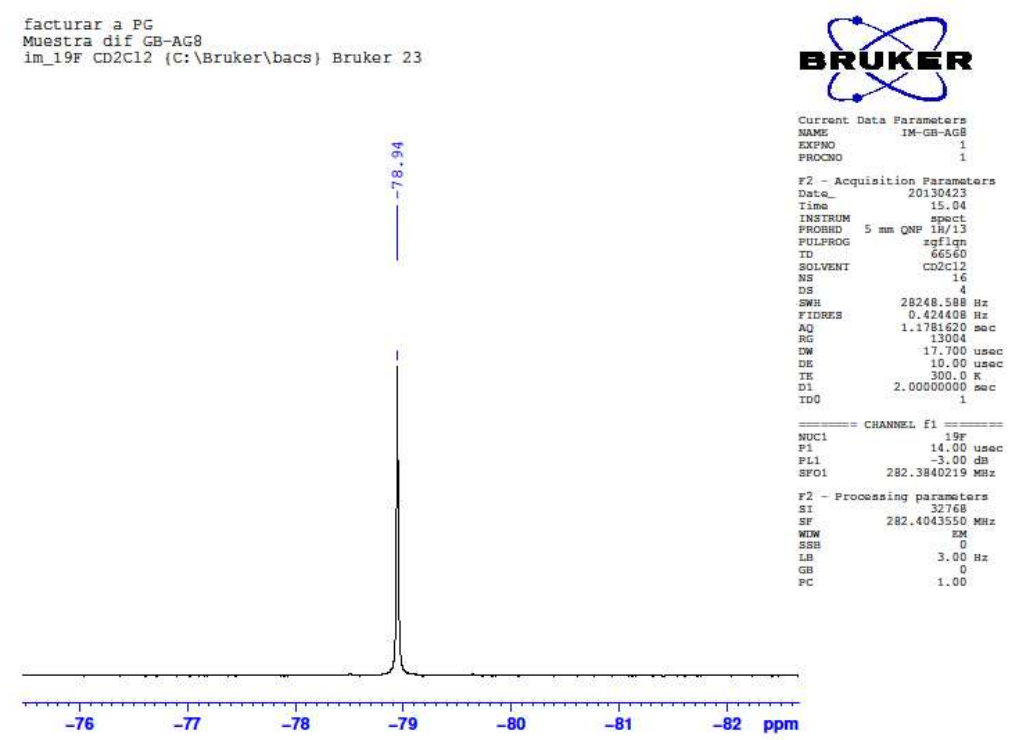

Complex 10. ${ }^{19} \mathrm{~F}$ RMN 298K 


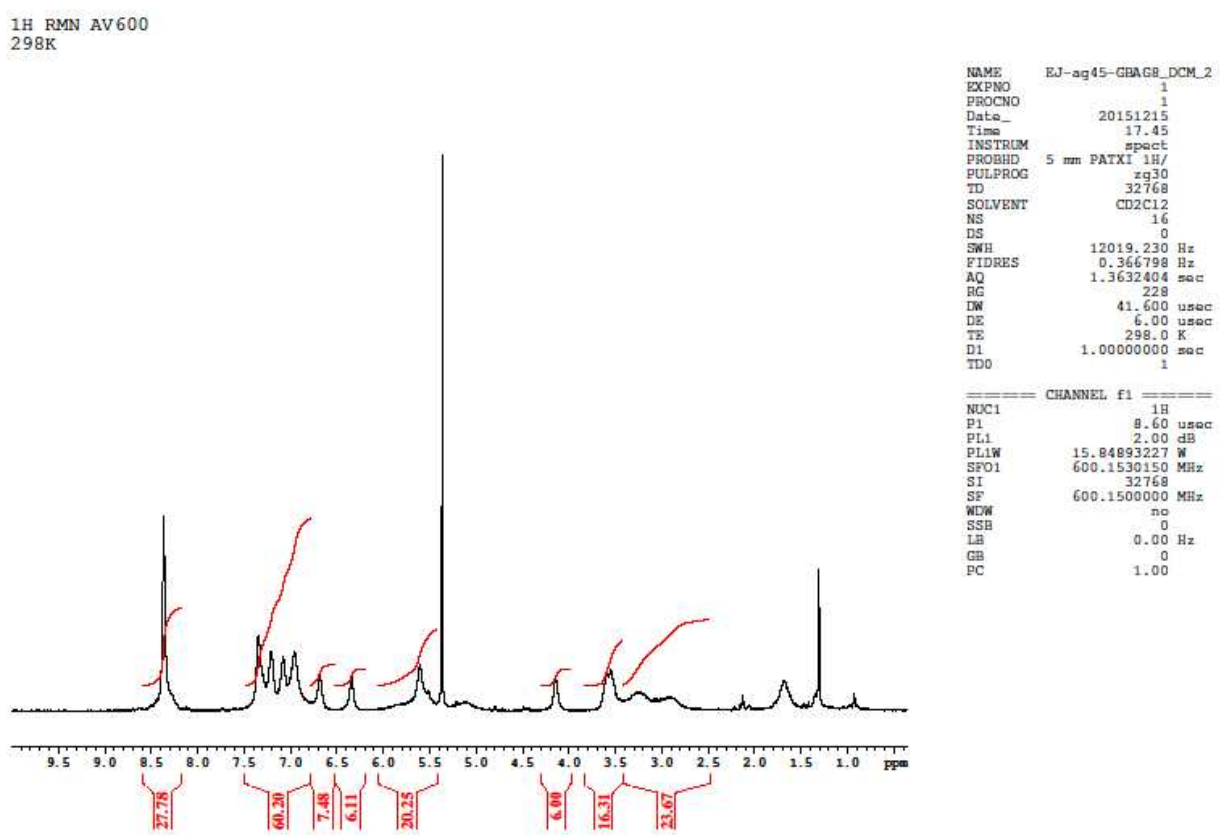

Complex 10. ${ }^{1} \mathrm{H}$ RMN $298 \mathrm{~K}$
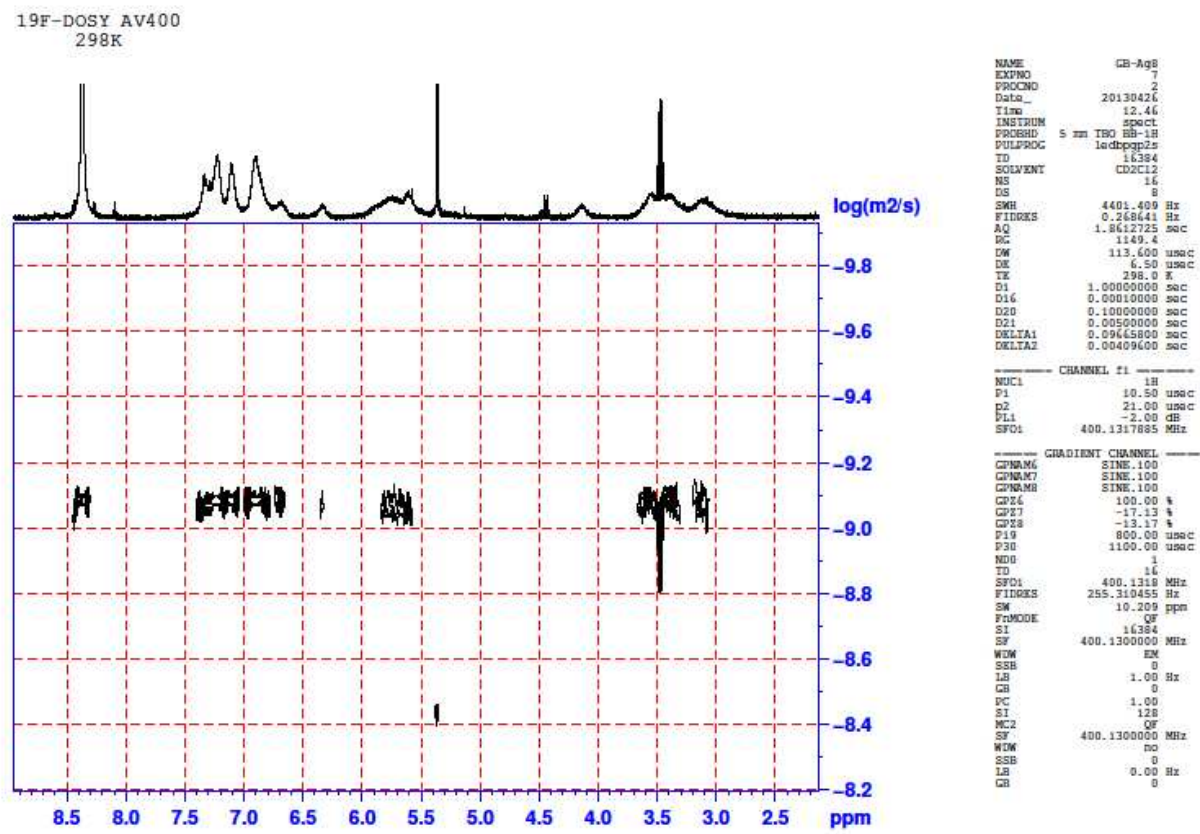

Complex 10. ${ }^{1} \mathrm{H}$ DOSY $298 \mathrm{~K}$ 
facturar a servicio

im_hoesy19F CD2C12 (C: \Bruker\bacs) Bruker 23
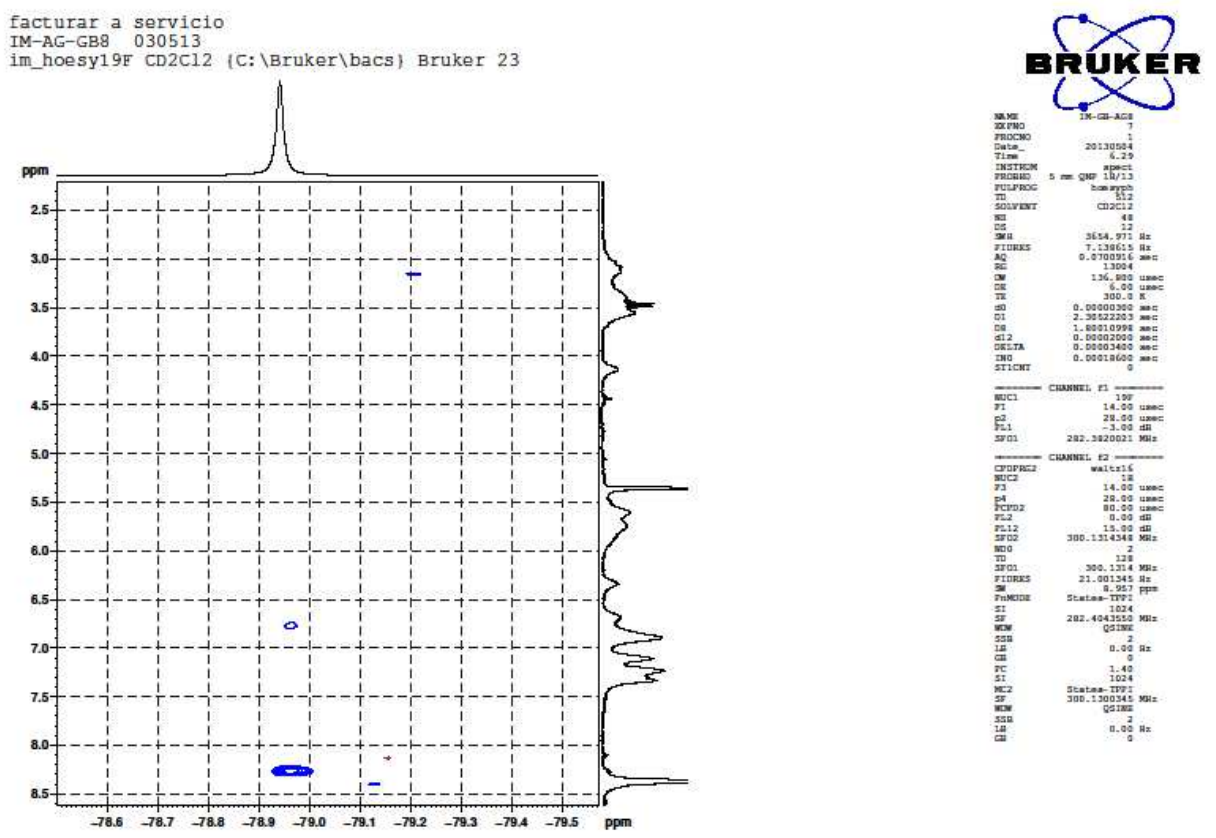

Complex 10. ${ }^{1} \mathrm{H}^{19} \mathrm{~F}$ HOESY $298 \mathrm{~K}$

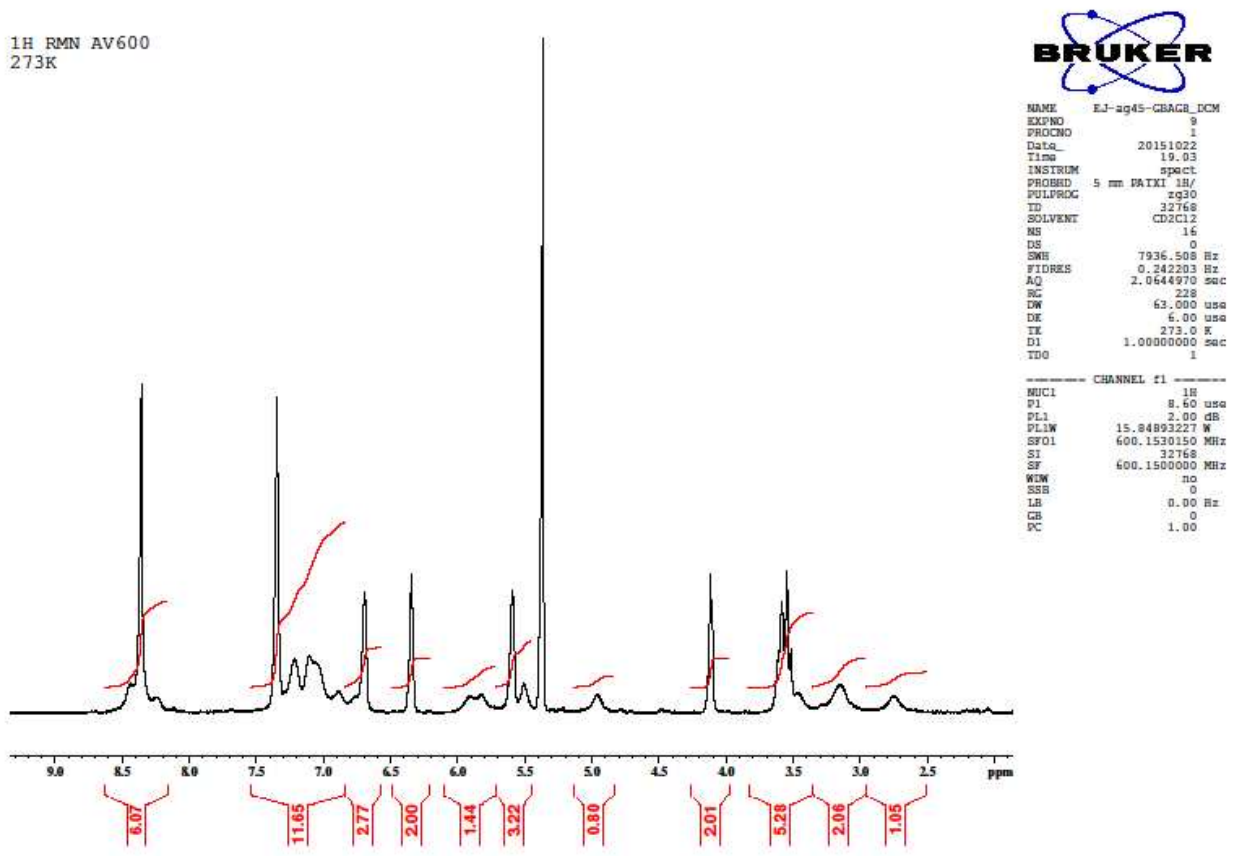

Complex 10. ${ }^{1} \mathrm{H} \mathrm{RMN} \mathrm{CD} \mathrm{Cl}_{2} 273 \mathrm{~K}$ 

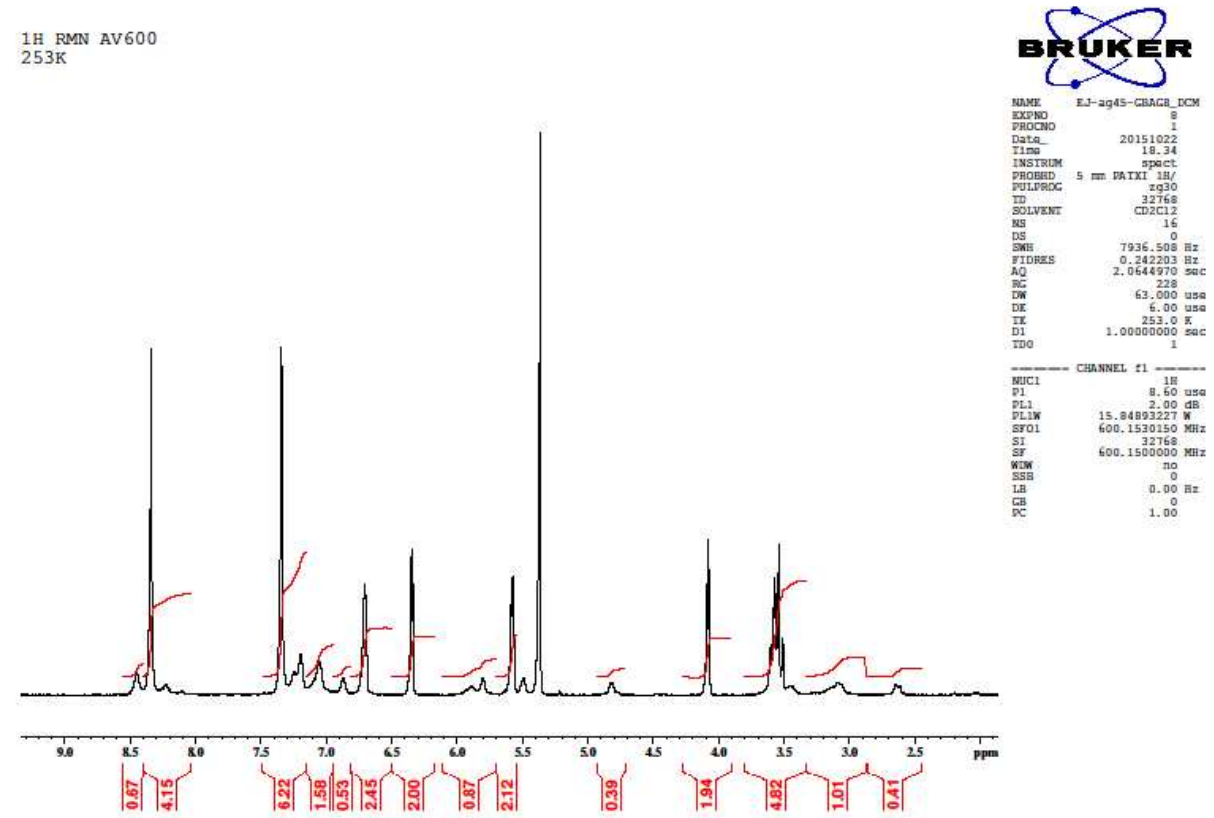

Complex 10. ${ }^{1} \mathrm{H} \mathrm{RMN} \mathrm{CD} \mathrm{Cl}_{2} 253 \mathrm{~K}$

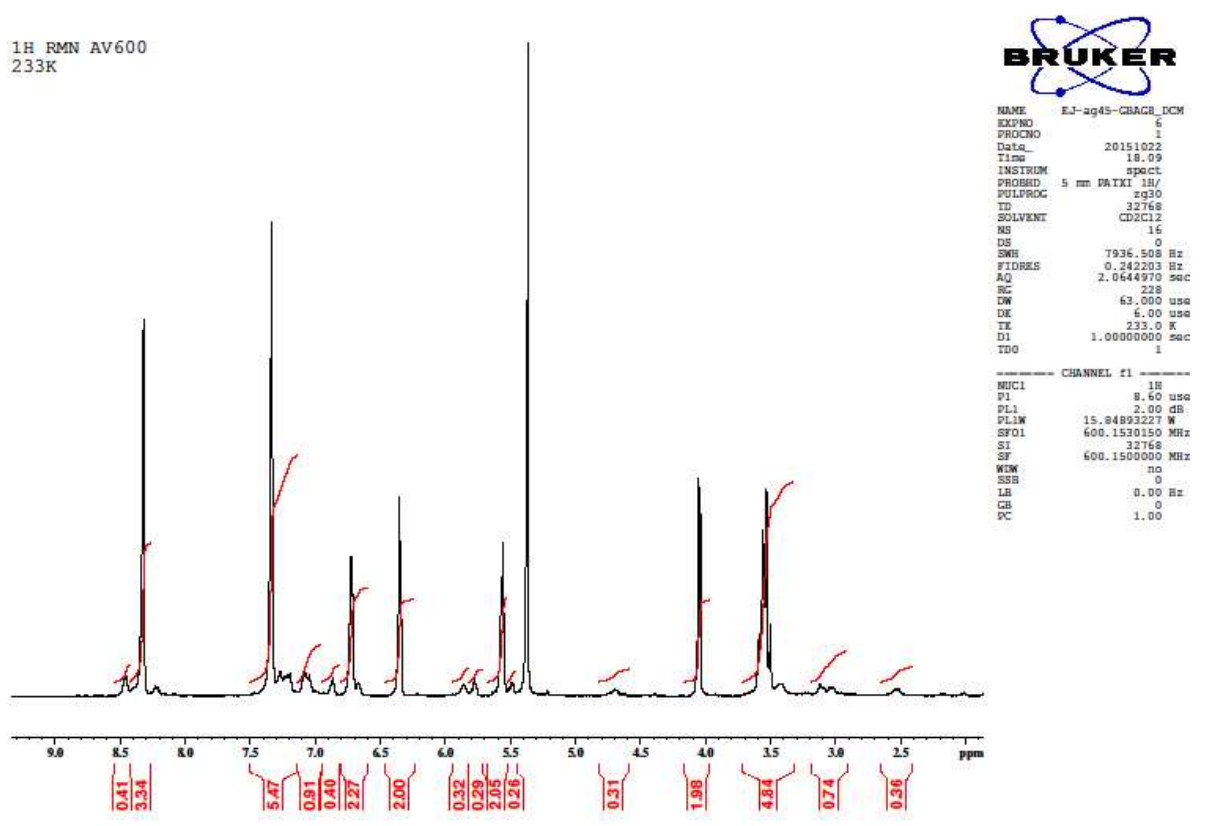

Complex 10. ${ }^{1} \mathrm{H} \mathrm{RMN} \mathrm{CD}{ }_{2} \mathrm{Cl}_{2} 233 \mathrm{~K}$ 


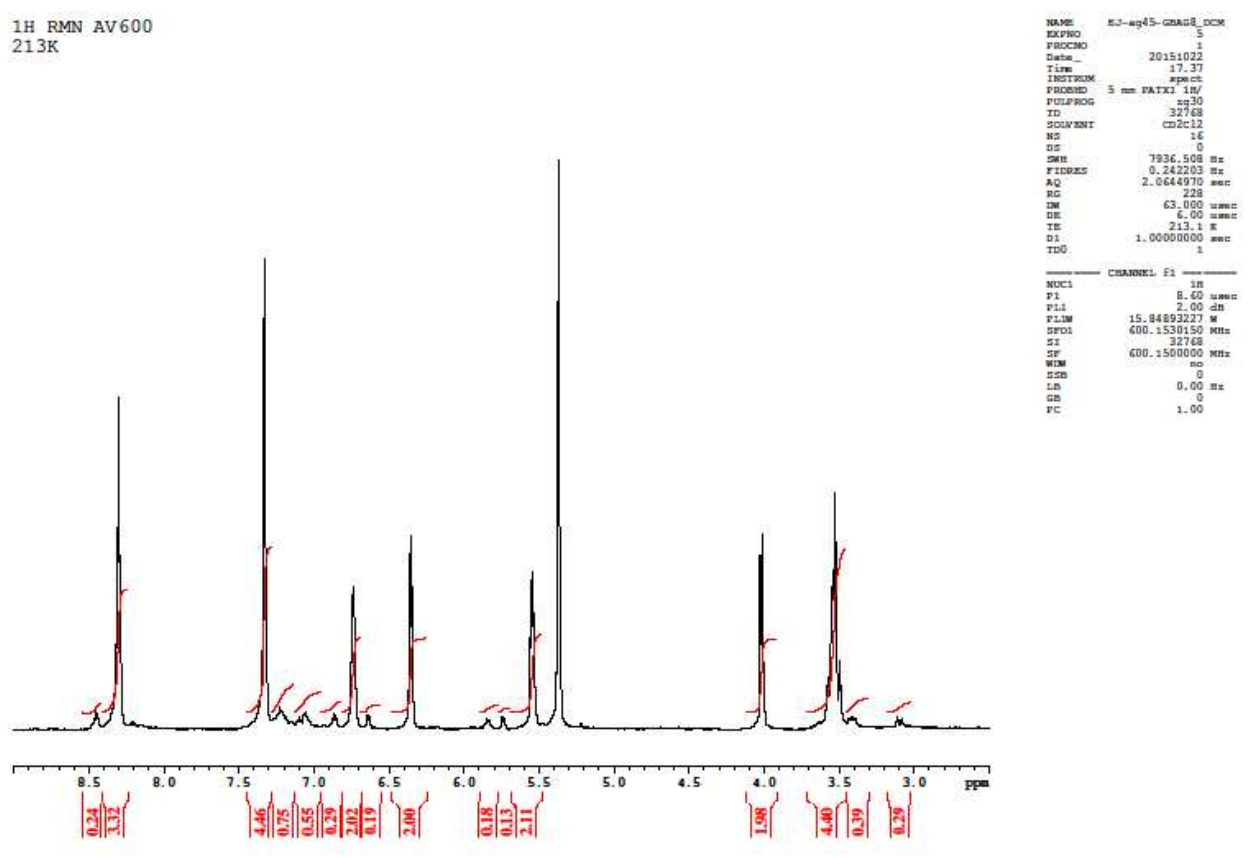

Complex 10. ${ }^{1} \mathrm{H} \mathrm{RMN} \mathrm{CD} \mathrm{Cl}_{2} 213 \mathrm{~K}$

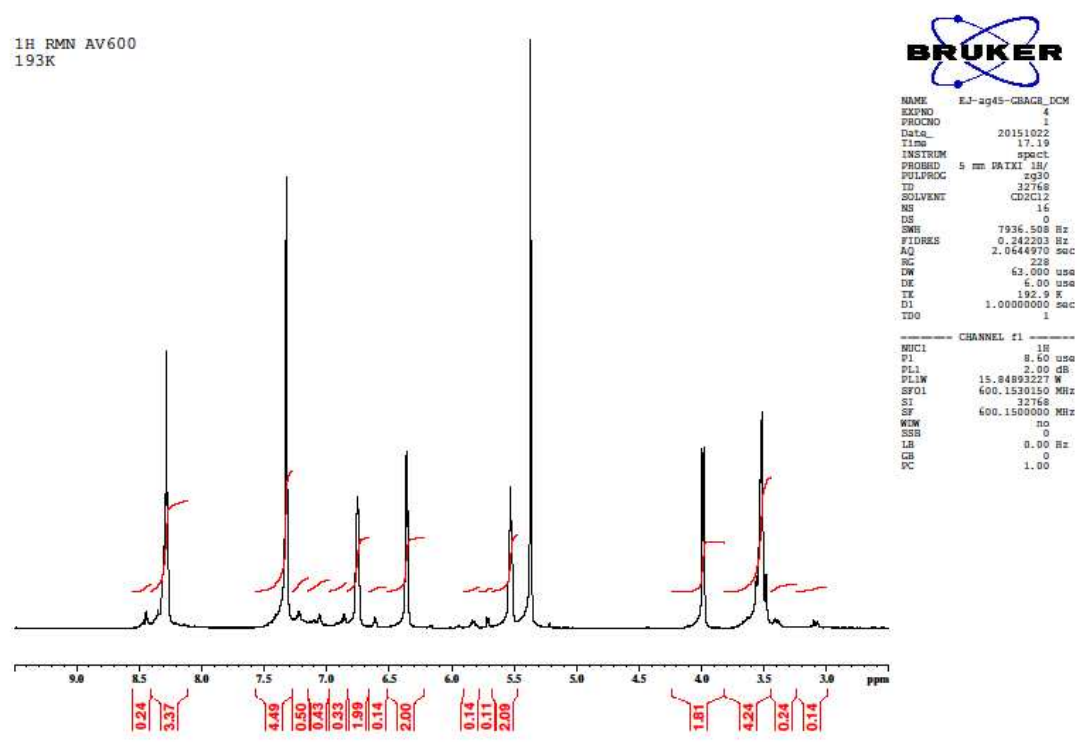

Complex 10. ${ }^{1} \mathrm{H} \mathrm{RMN} \mathrm{CD}{ }_{2} \mathrm{Cl}_{2} 193 \mathrm{~K}$ 


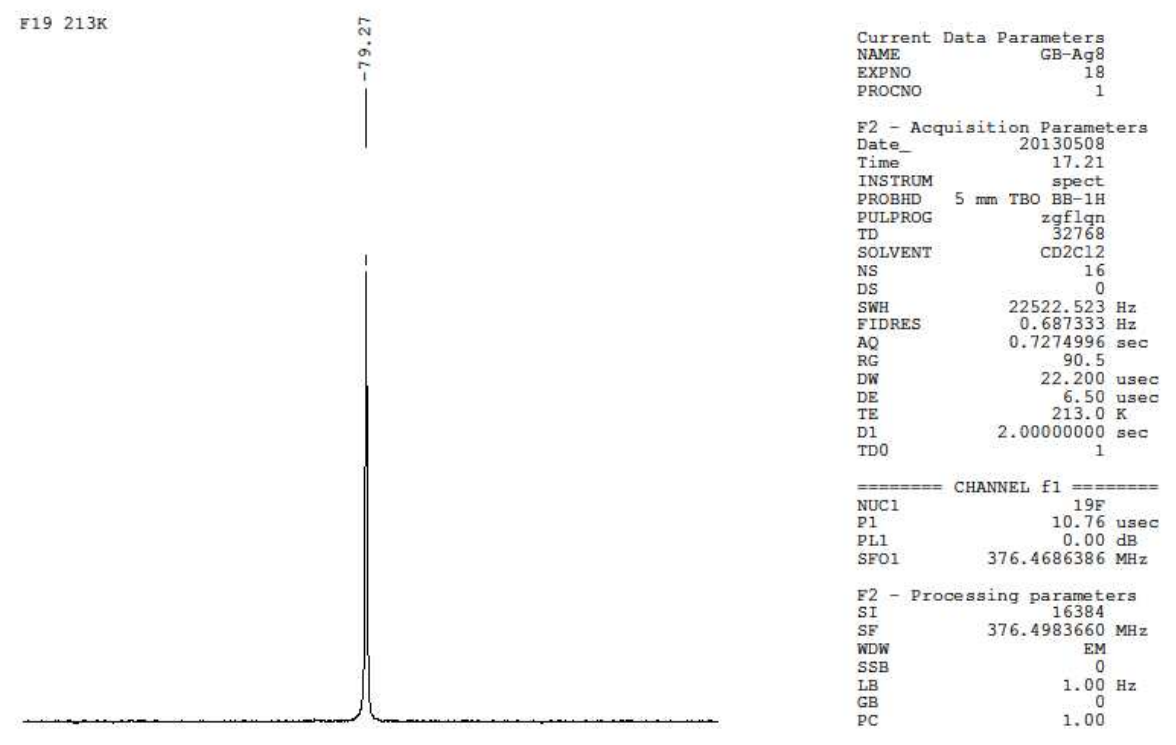

Complex 10. ${ }^{19} \mathrm{~F}$ RMN $\mathrm{CD}_{2} \mathrm{Cl}_{2} 213 \mathrm{~K}$
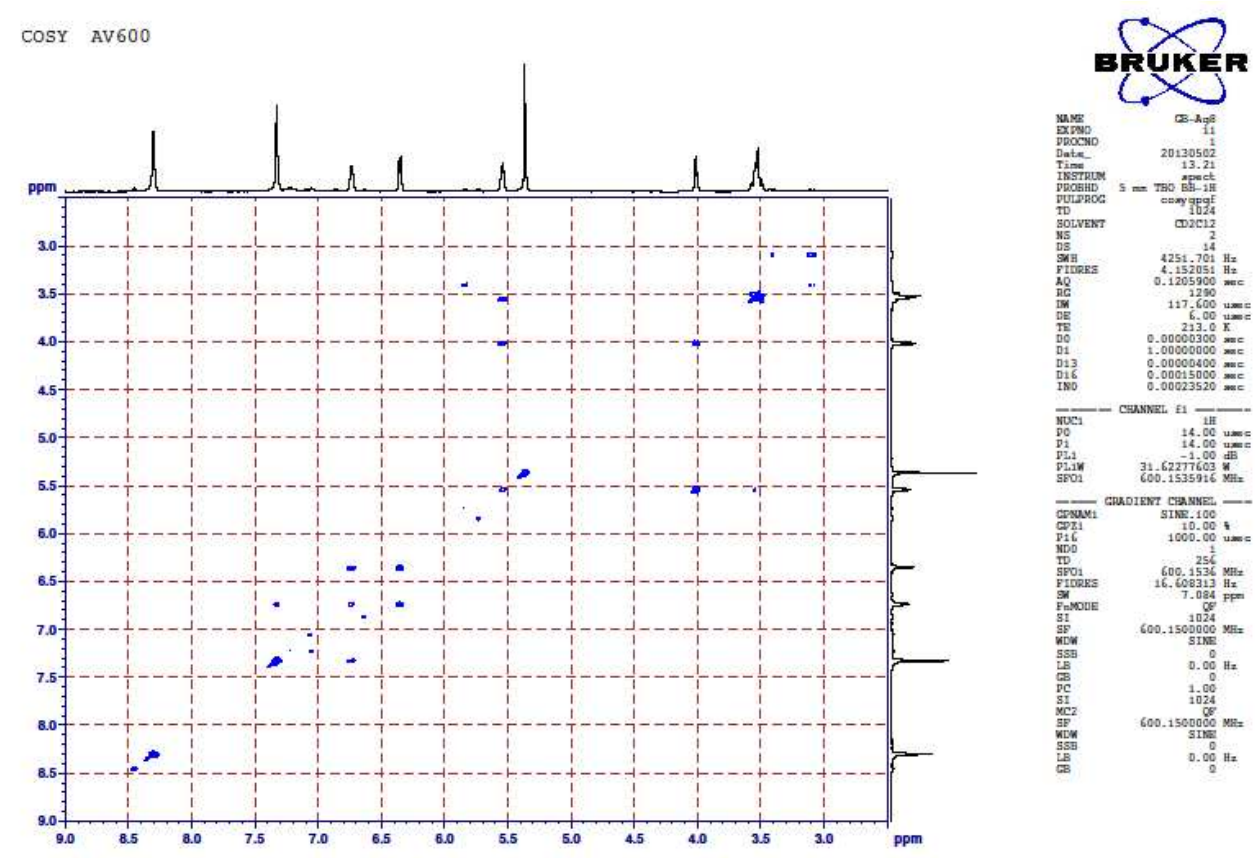

Complex 10. COSY 213K 

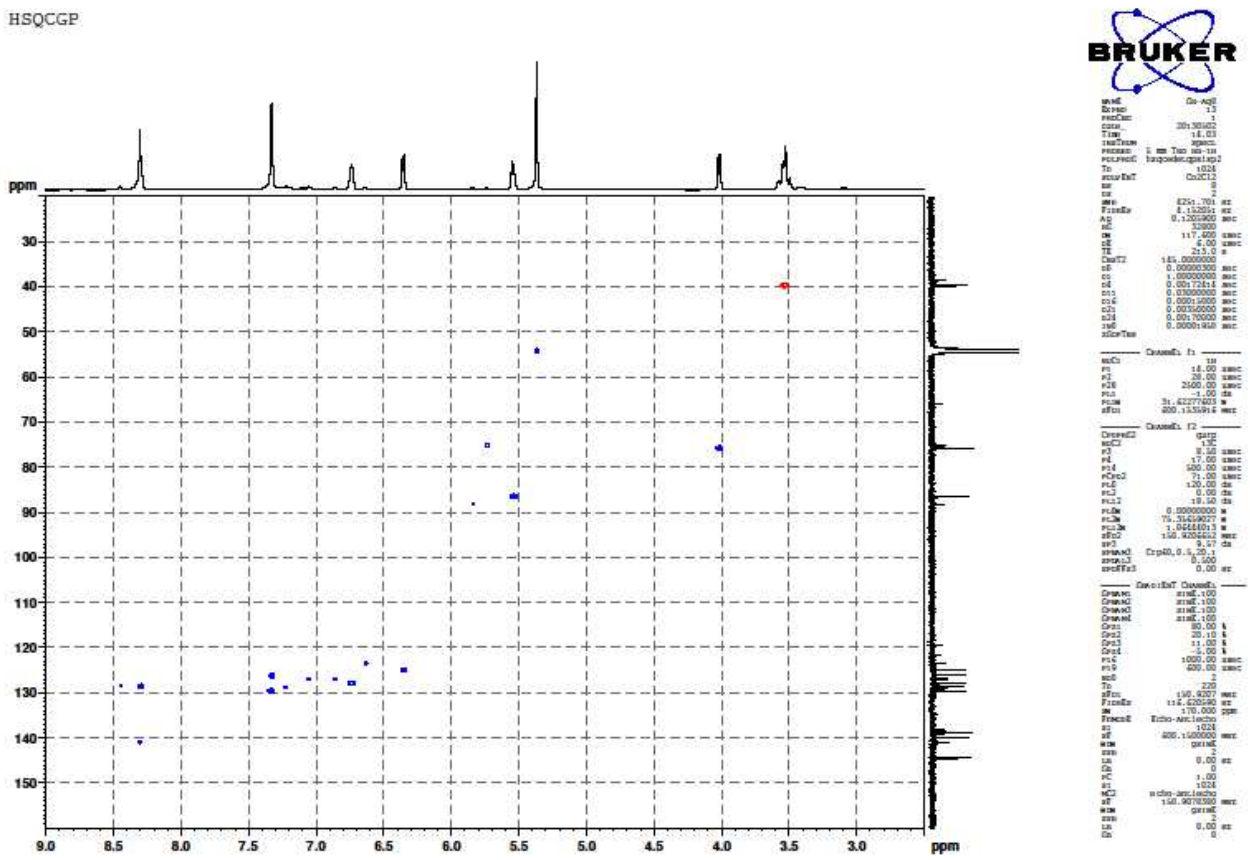

Complex 10. ${ }^{1} \mathrm{H}^{13} \mathrm{C}$ HSQC $213 \mathrm{~K}$
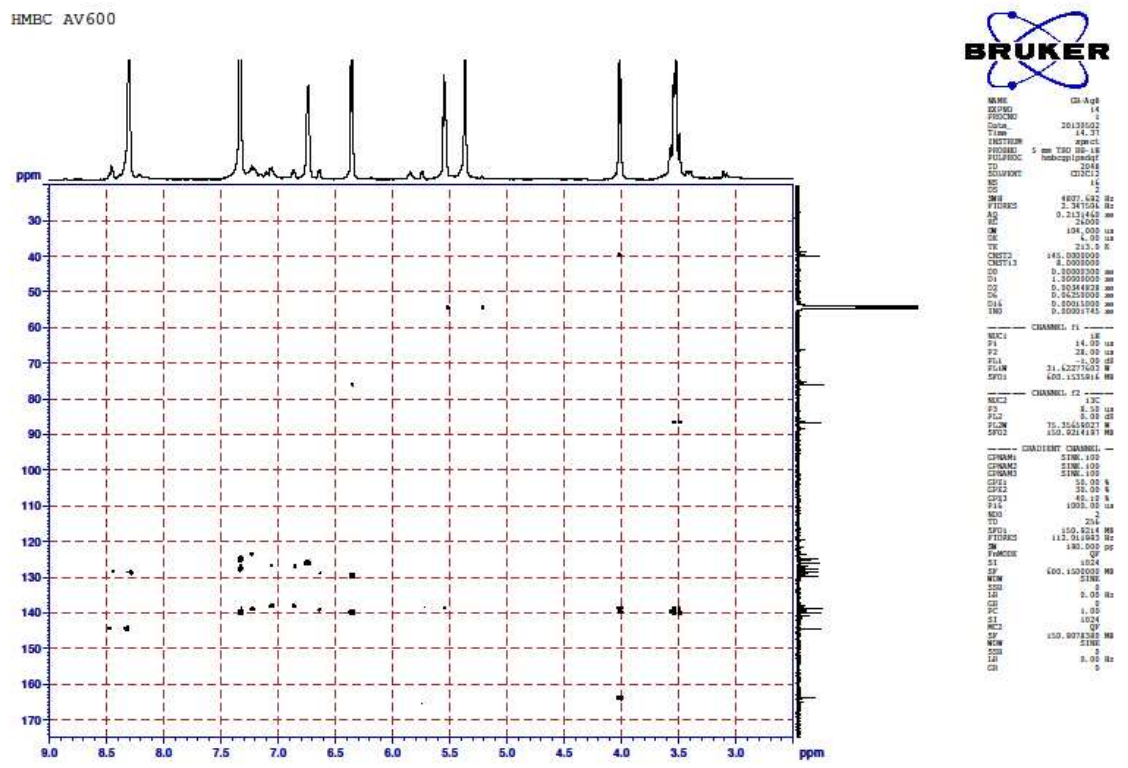

Complex 10. ${ }^{1} \mathrm{H}^{13} \mathrm{C}$ HMBC $213 \mathrm{~K}$ 


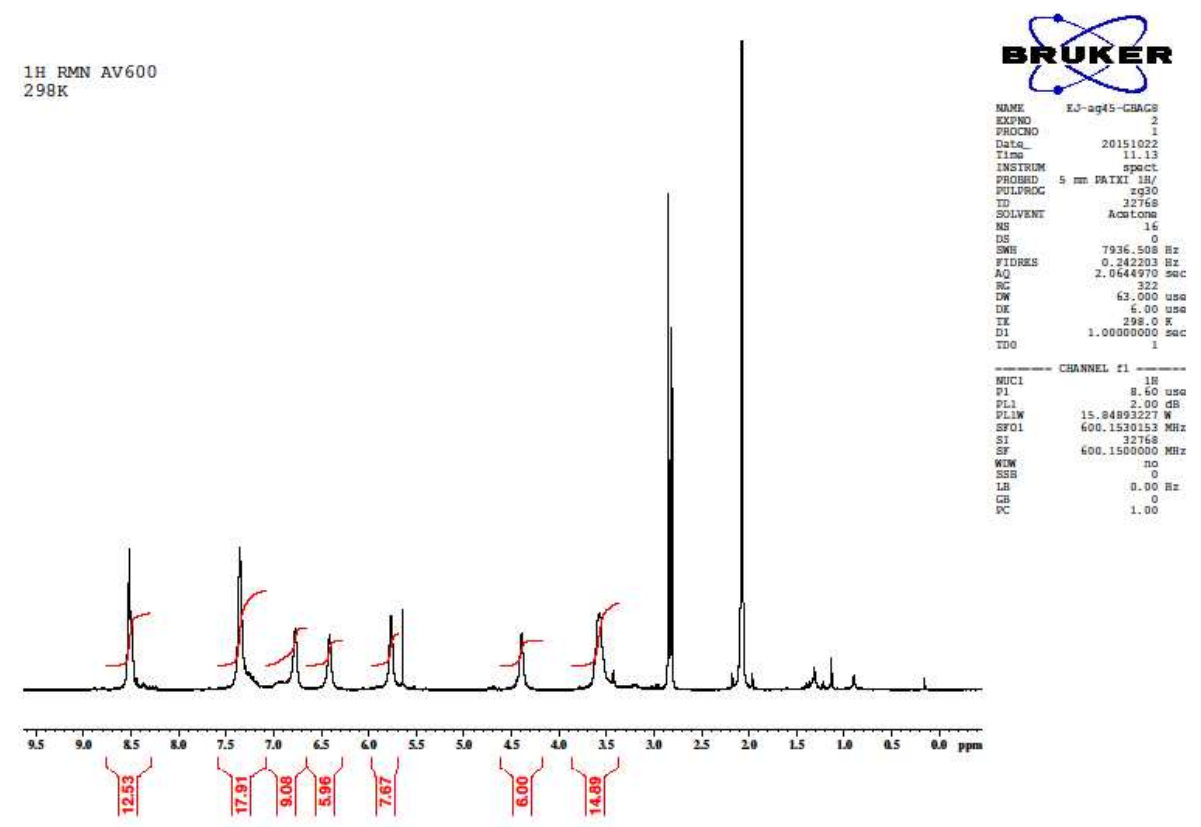

Complex 10. ${ }^{1}$ H RMN 298K Acetone- $d_{6}$

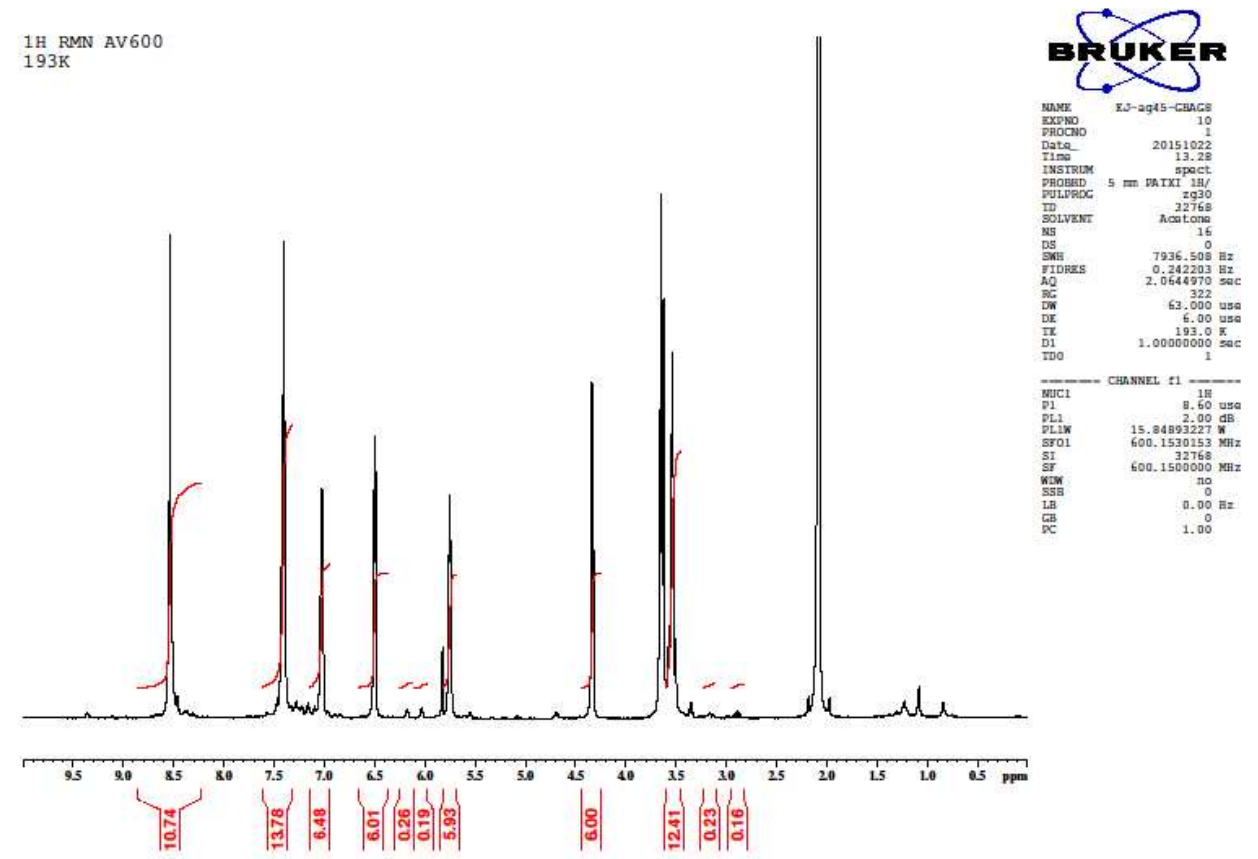

Complex 10. ${ }^{1} \mathrm{H}$ RMN 193K Acetone- $d_{6}$ 


\section{Complex 12}

$\begin{array}{ll}\text { facturar a } & \text { pg } \\ \text { IM-GB-Ag18 } & \text { PF6 }\end{array}$

p31.200 CD2C12 (C: \Bruker \bacs) Bruker 26
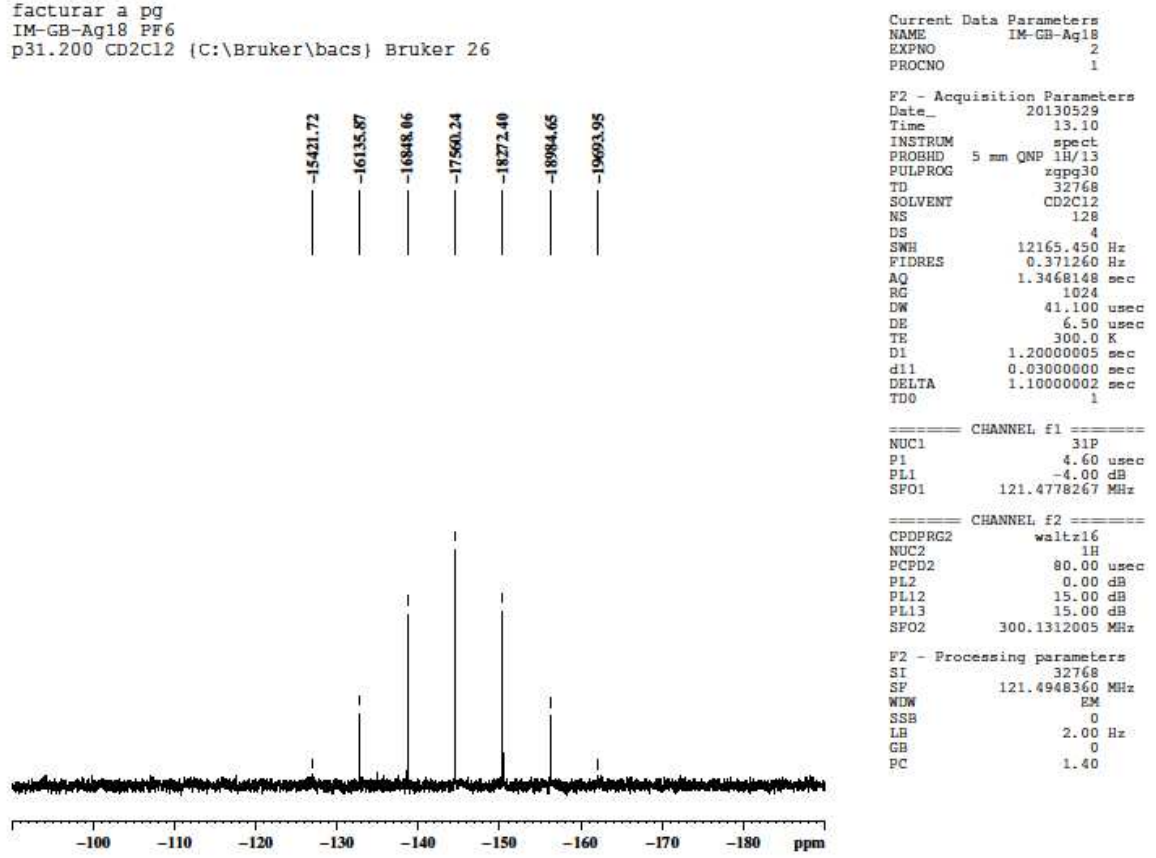

Complex 12. ${ }^{31} \mathrm{P}$ RMN 298K

facturar a pg
IM-GB-Ag18 PF 6

19 CD2C12 (C: \Bruker\bacs) Bruker 26
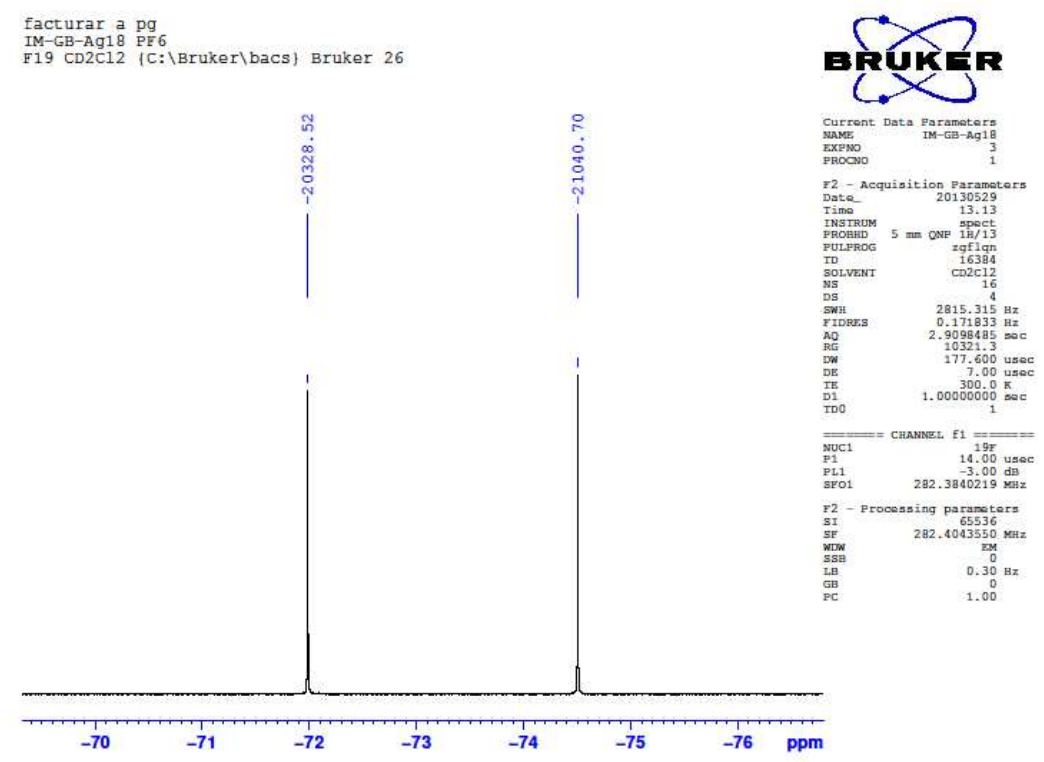

Complex 12. ${ }^{19} \mathrm{~F}$ RMN 298K 

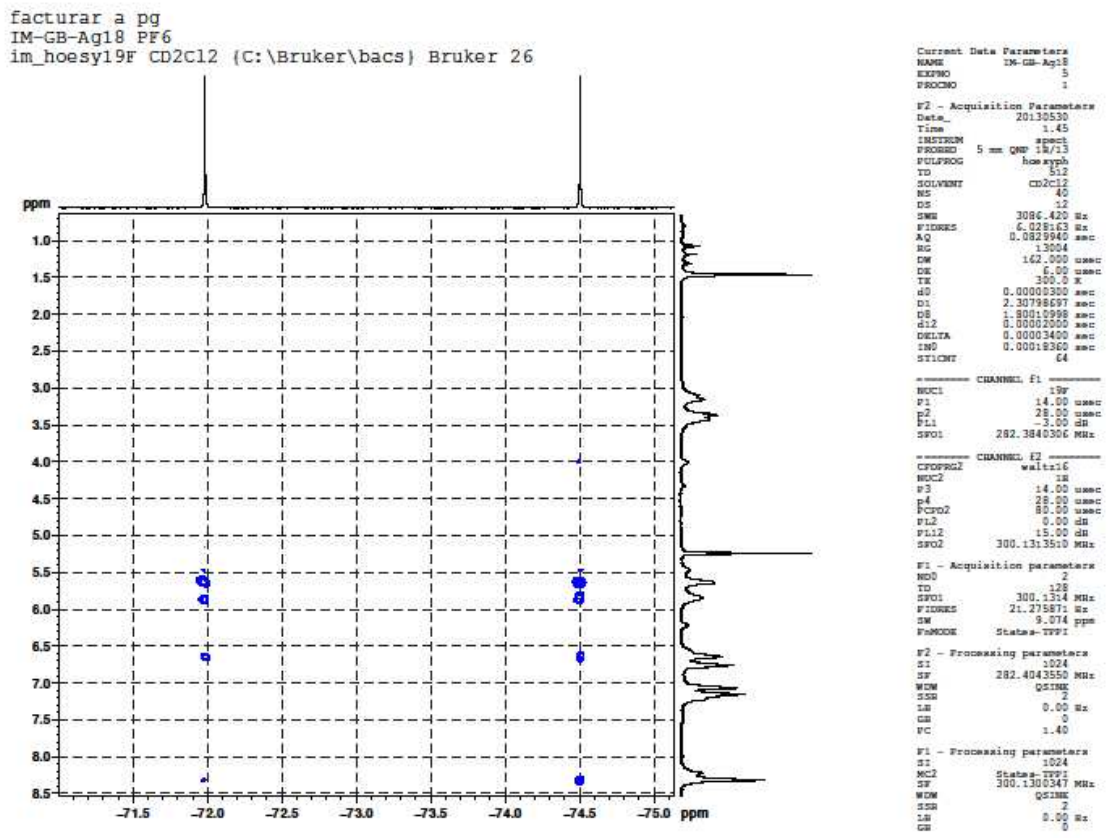

Complex 12. ${ }^{1} \mathrm{H}^{19} \mathrm{~F}$ HOESY $298 \mathrm{~K}$
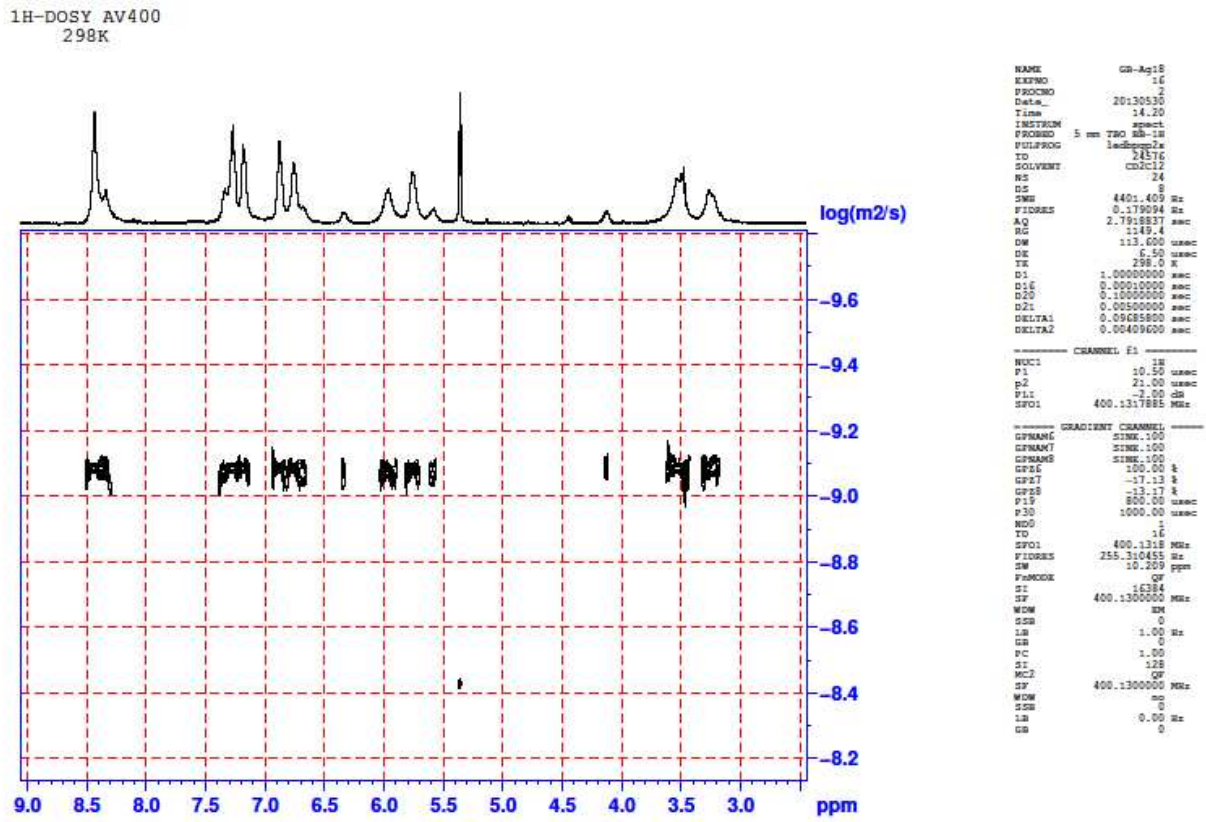

Complex 12. ${ }^{1}$ H DOSY $298 \mathrm{~K}$ 


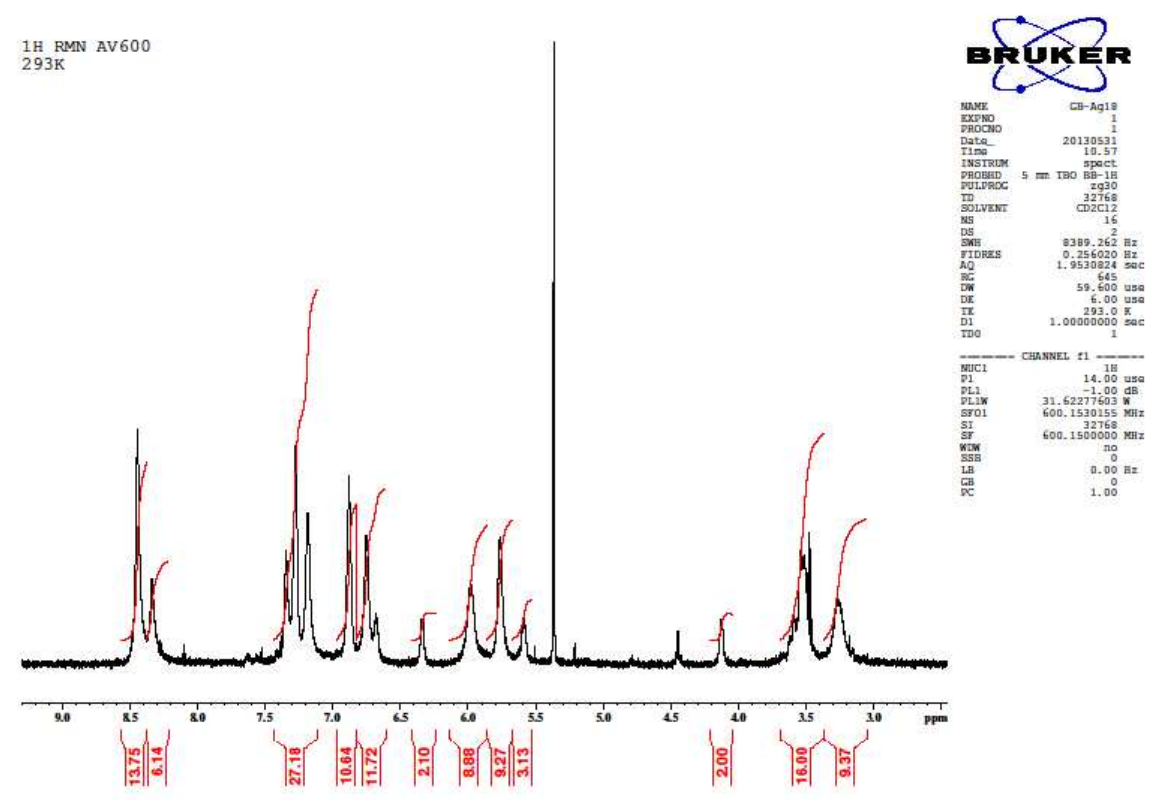

Complex 12. ${ }^{1} \mathrm{H}$ RMN $293 \mathrm{~K}$

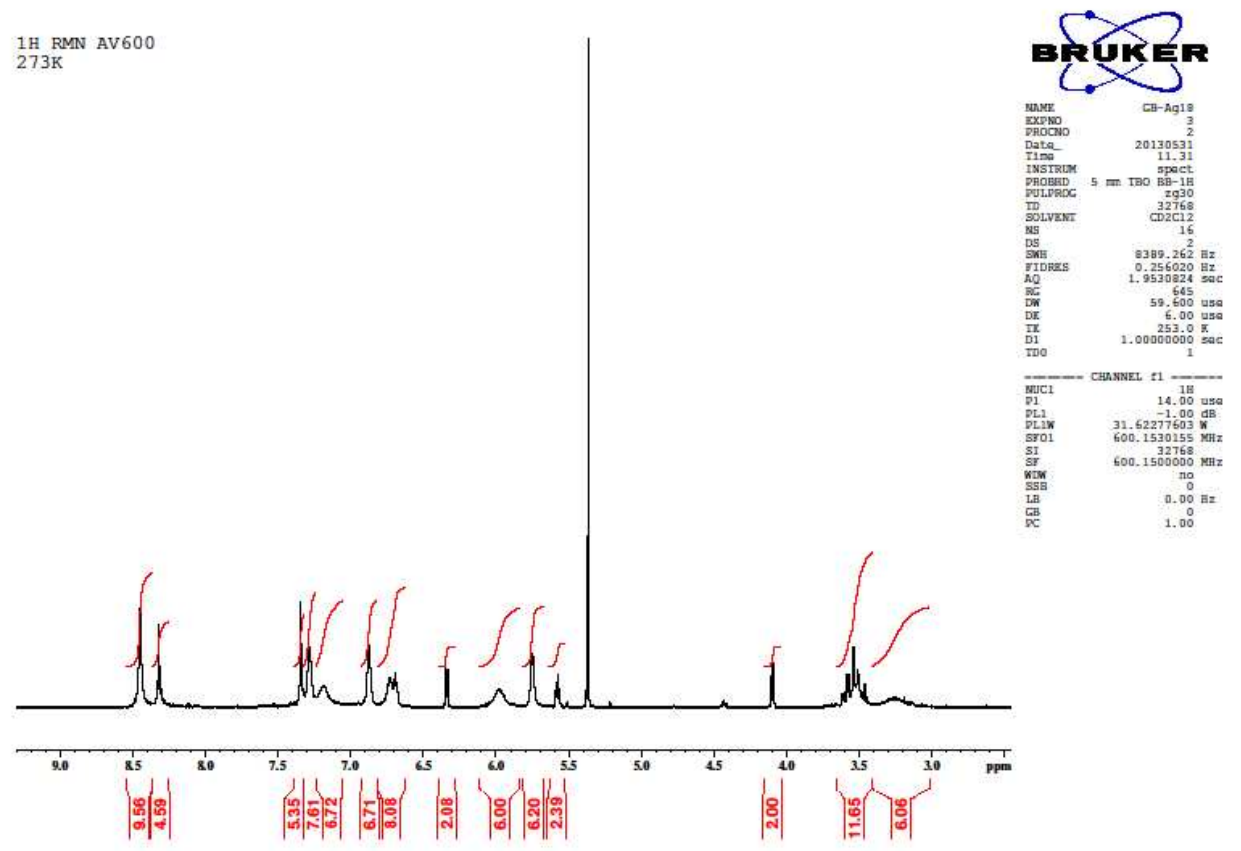

Complex 12. ${ }^{1} \mathrm{H}$ RMN $273 \mathrm{~K}$ 


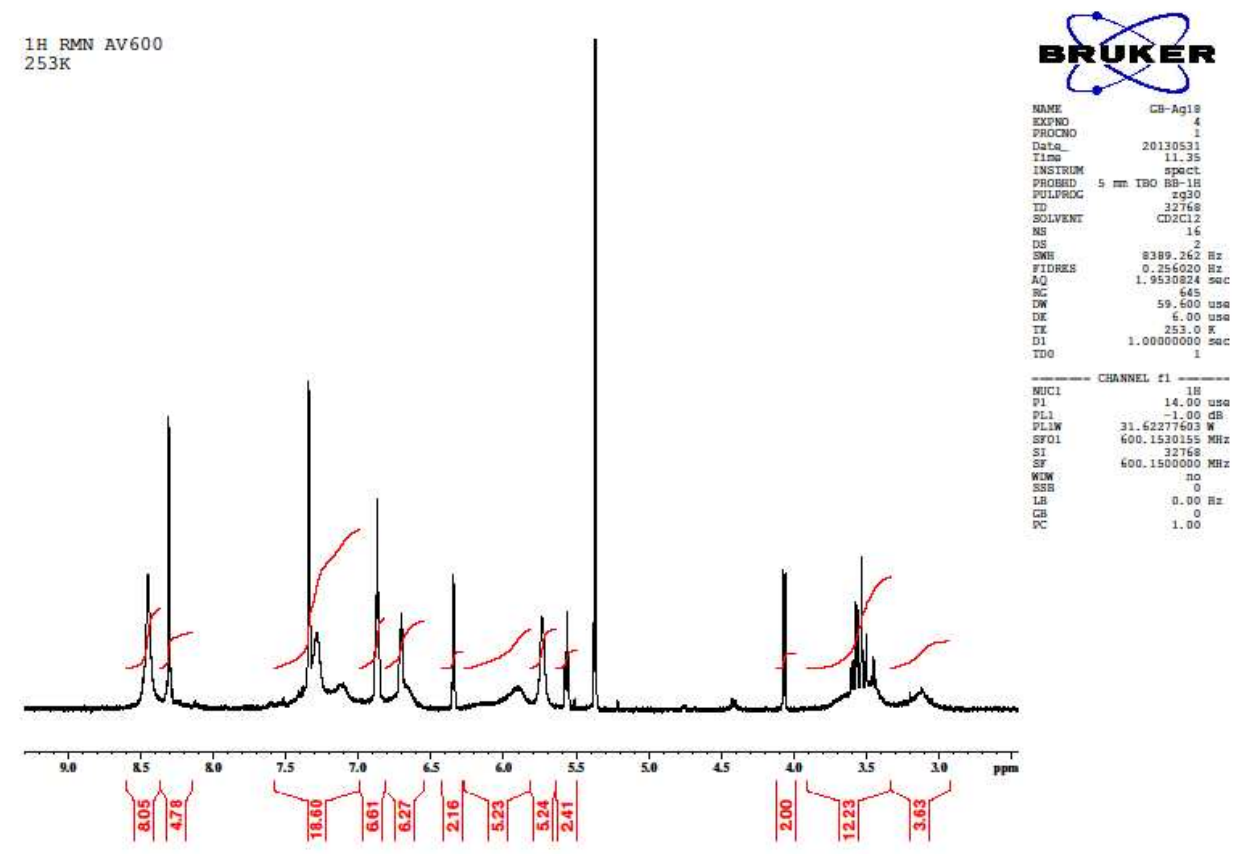

Complex 12. ${ }^{1} \mathrm{H}$ RMN $253 \mathrm{~K}$

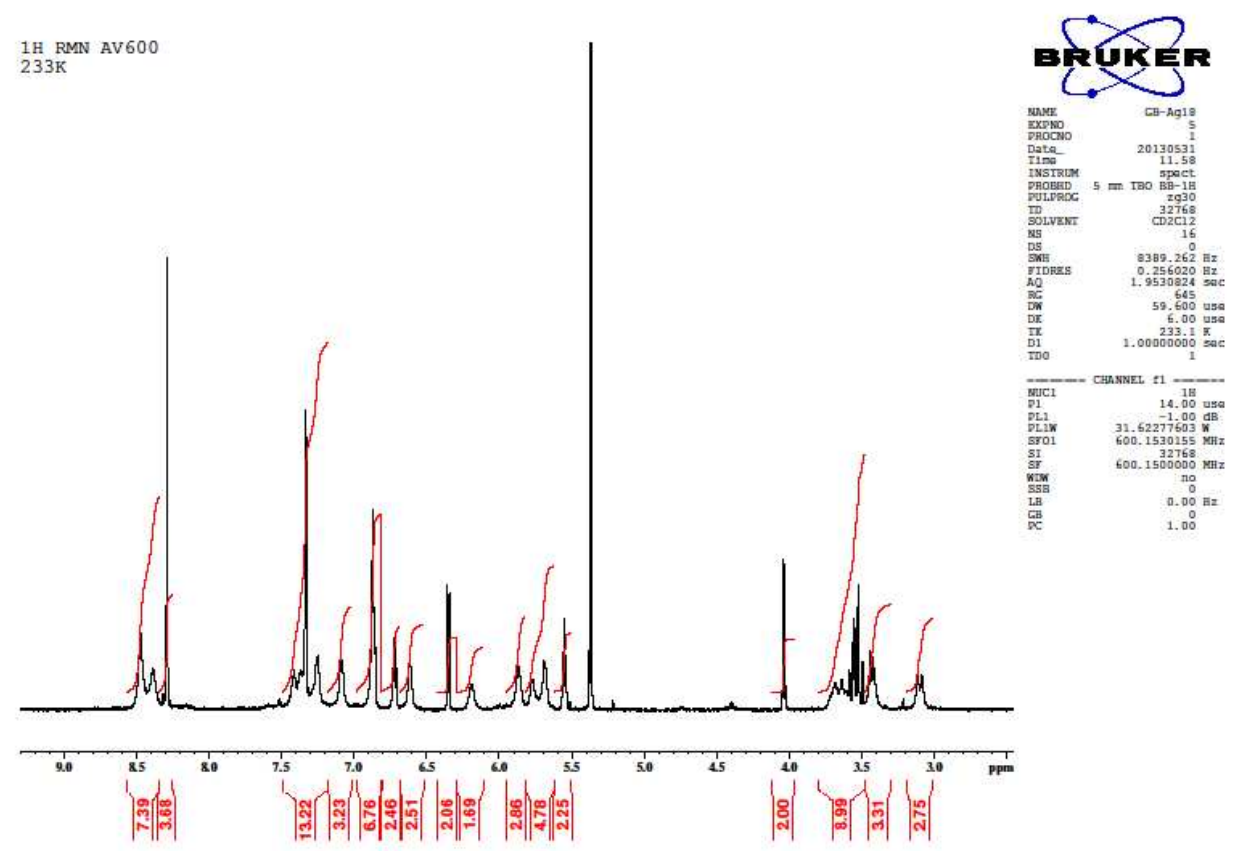

Complex 12. ${ }^{1} \mathrm{H}$ RMN $233 \mathrm{~K}$ 


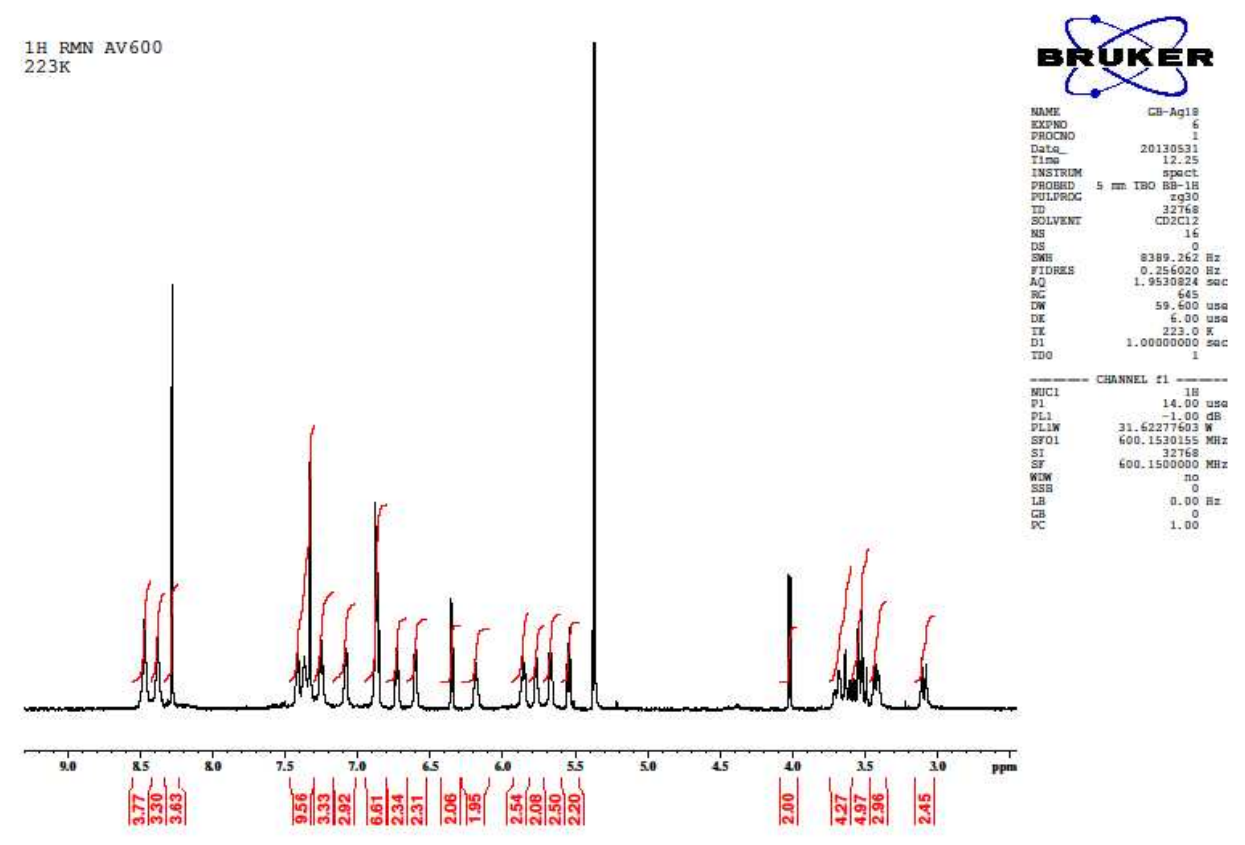

Complex 12. ${ }^{1} \mathrm{H}$ RMN $223 \mathrm{~K}$

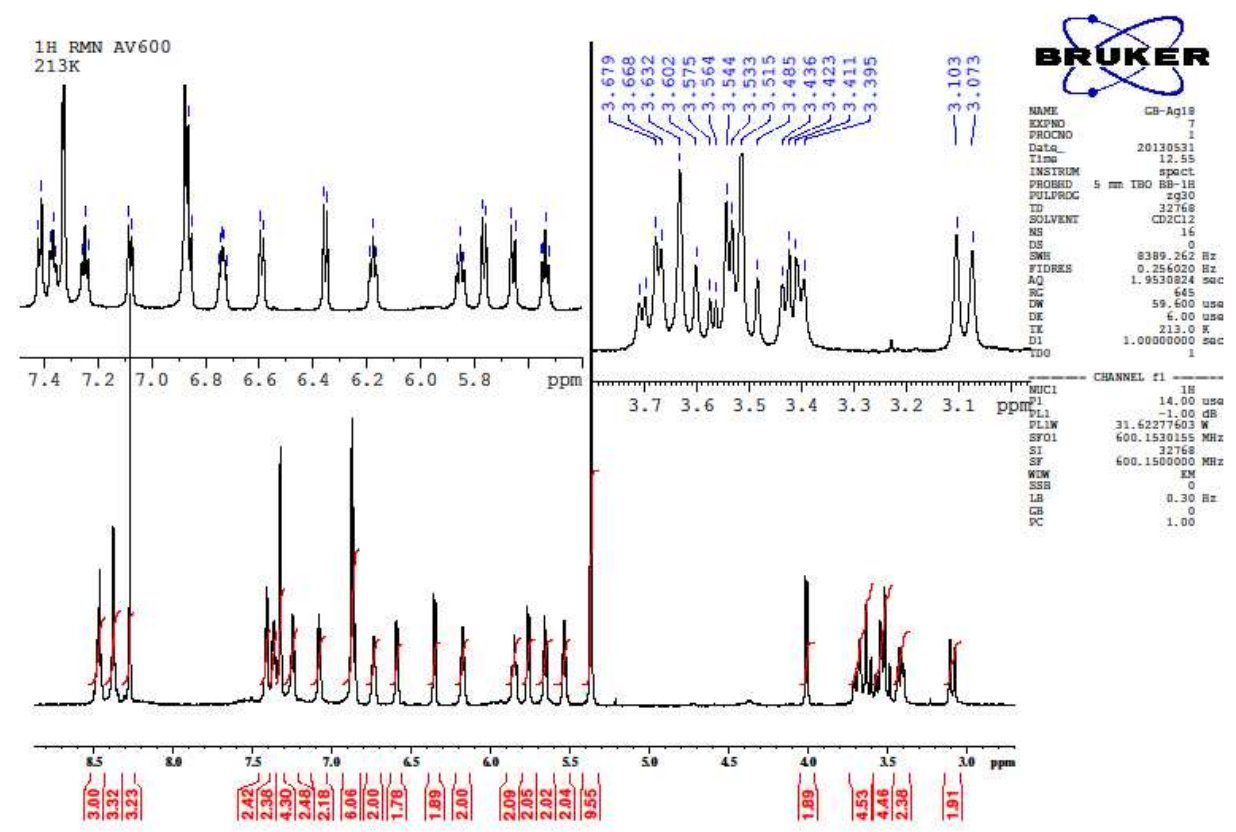

Complex 12. ${ }^{1} \mathrm{H}$ RMN $213 \mathrm{~K}$ 


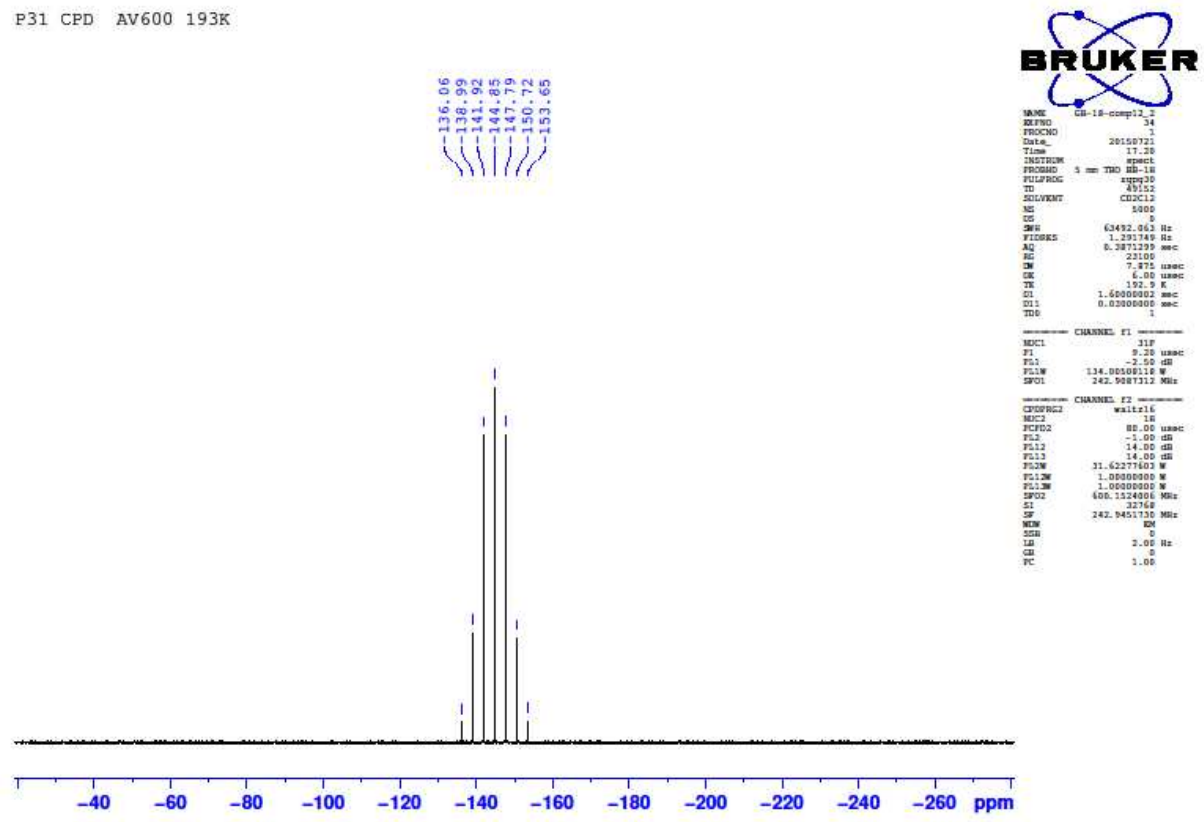

Complex 12. ${ }^{31} \mathrm{P}$ RMN 193K

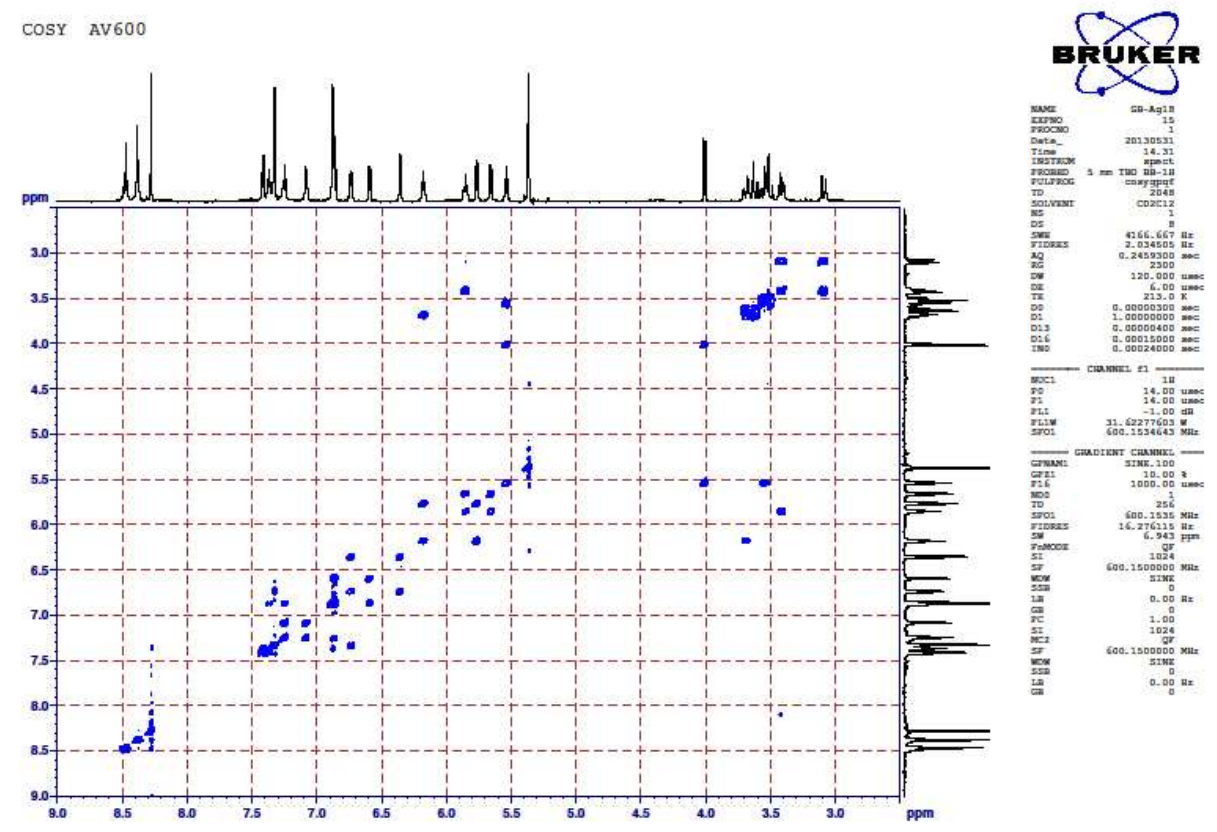

Complex 12. COSY $213 \mathrm{~K}$ 


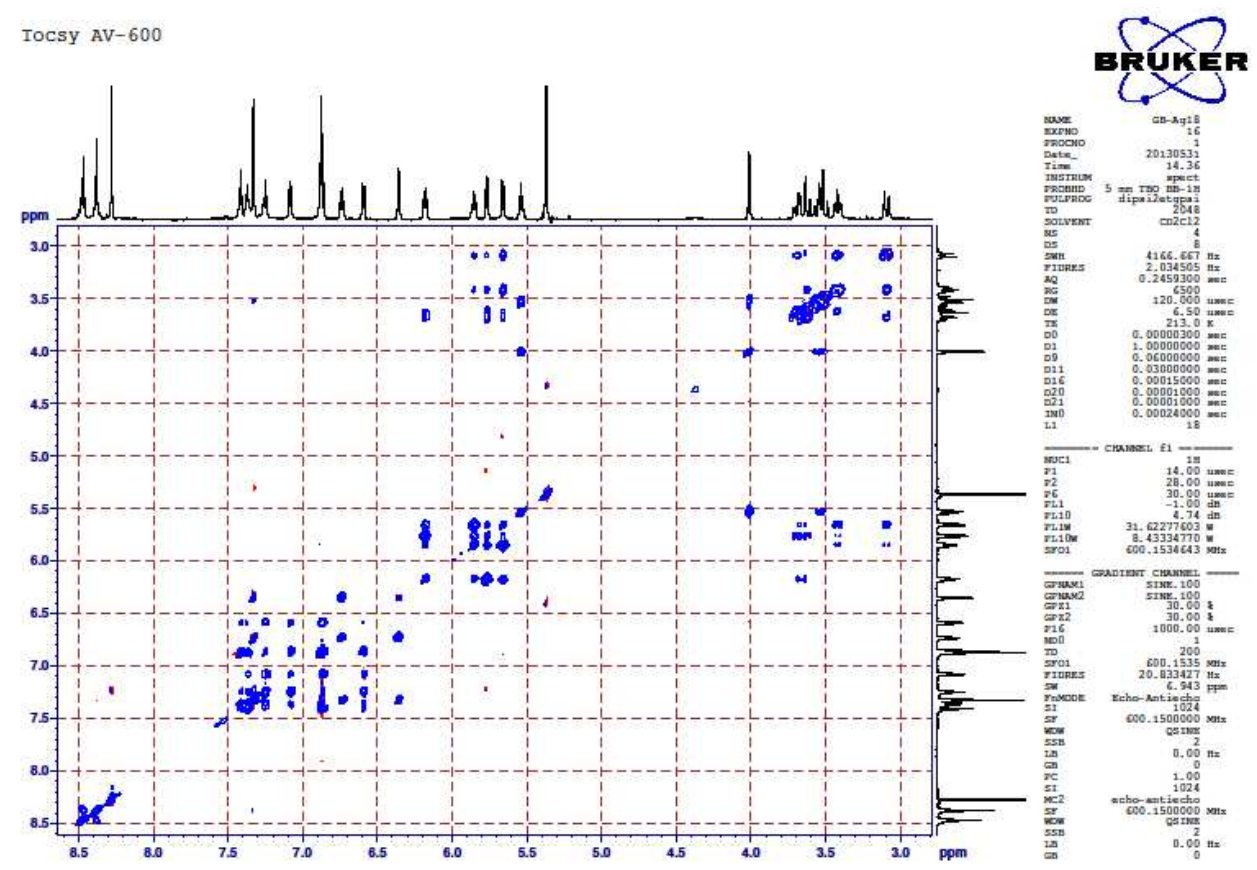

Complex 12. TOCSY 213K
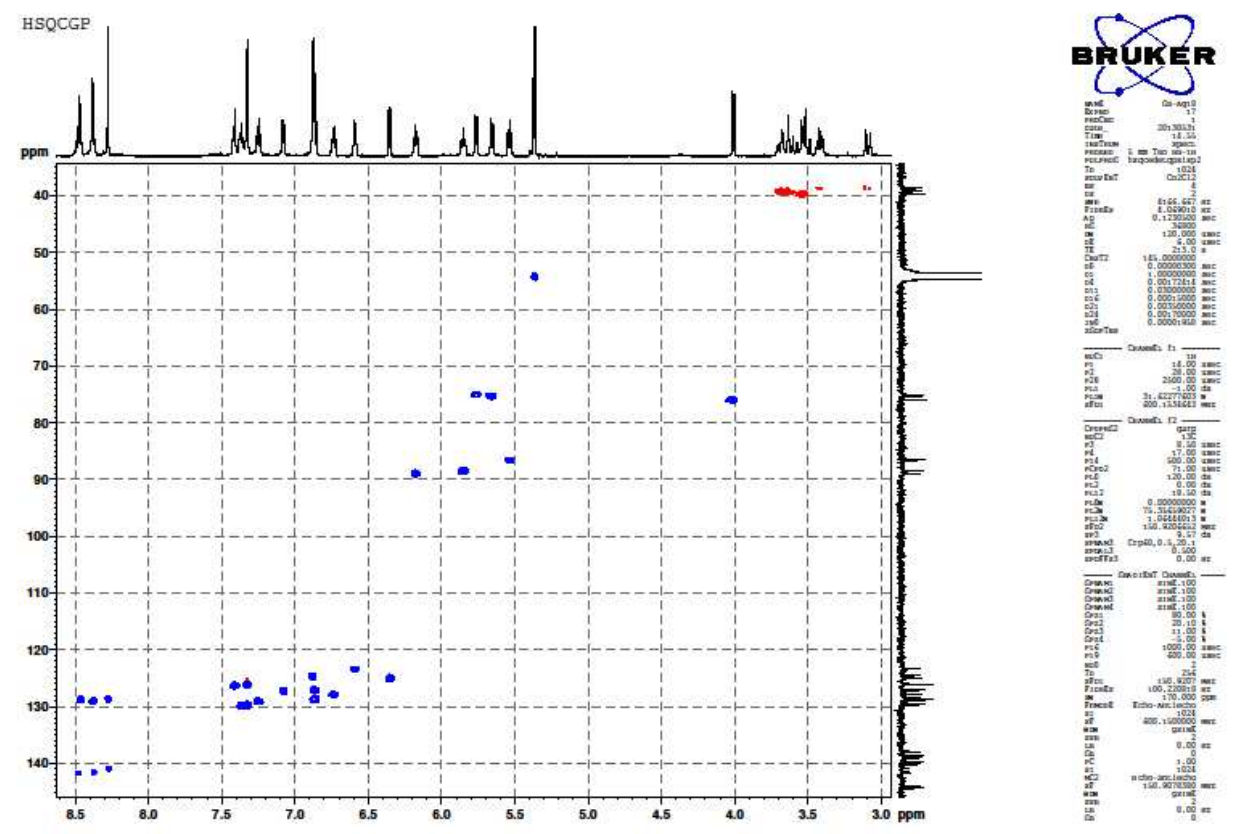

Complex 12. ${ }^{1} \mathrm{H}^{13} \mathrm{C}$ HSQC $213 \mathrm{~K}$ 


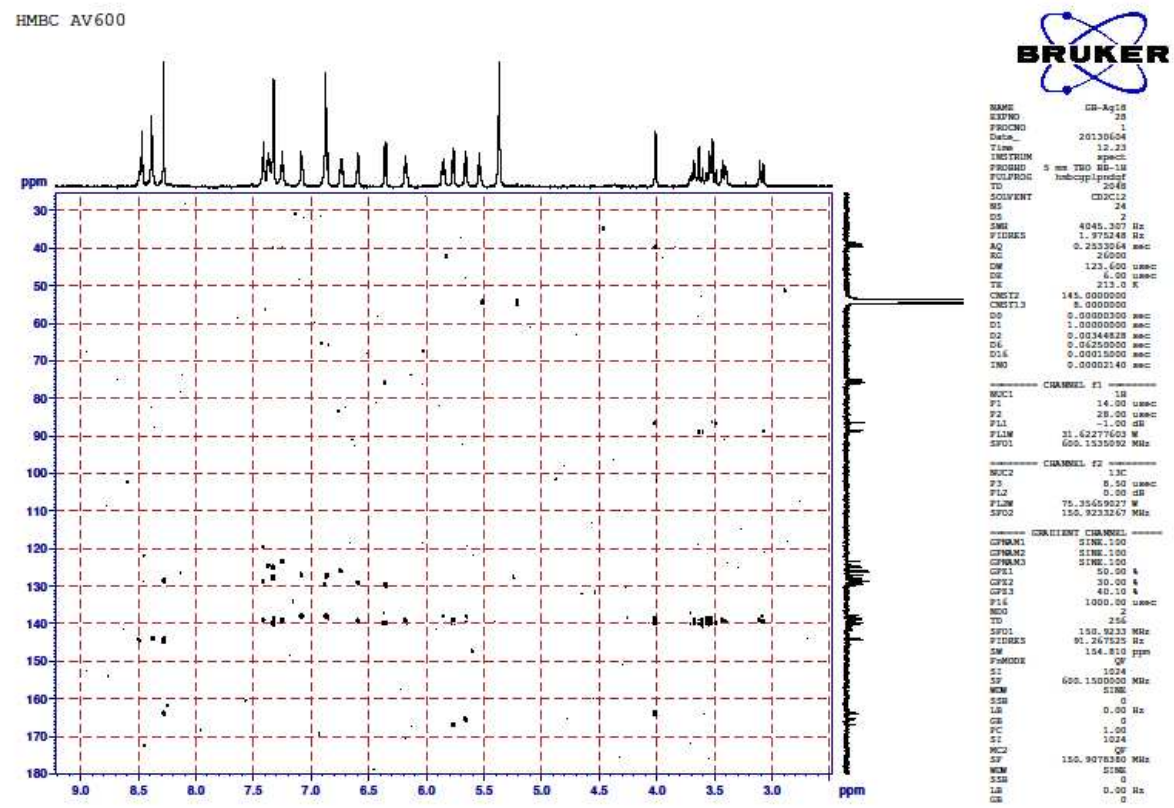

Complex 12. ${ }^{1} \mathrm{H}^{13} \mathrm{C}$ HMB $213 \mathrm{~K}$

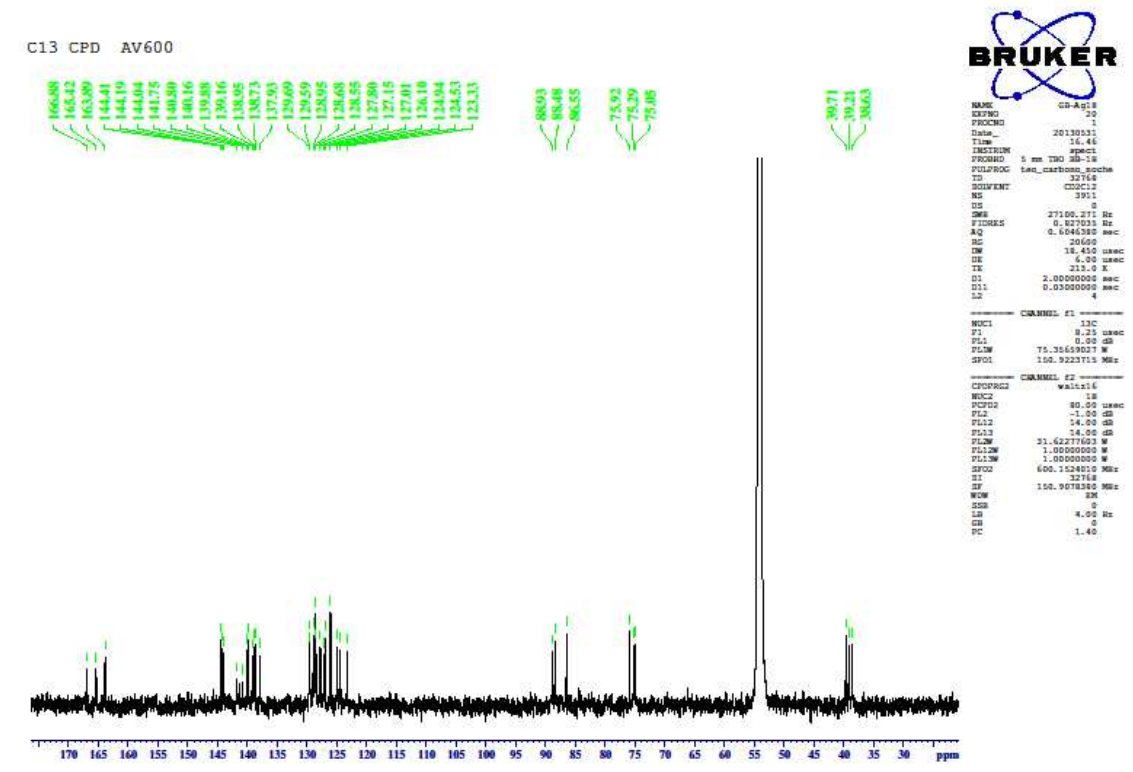

Complex 12. ${ }^{13} \mathrm{C}$ RMN 213K 

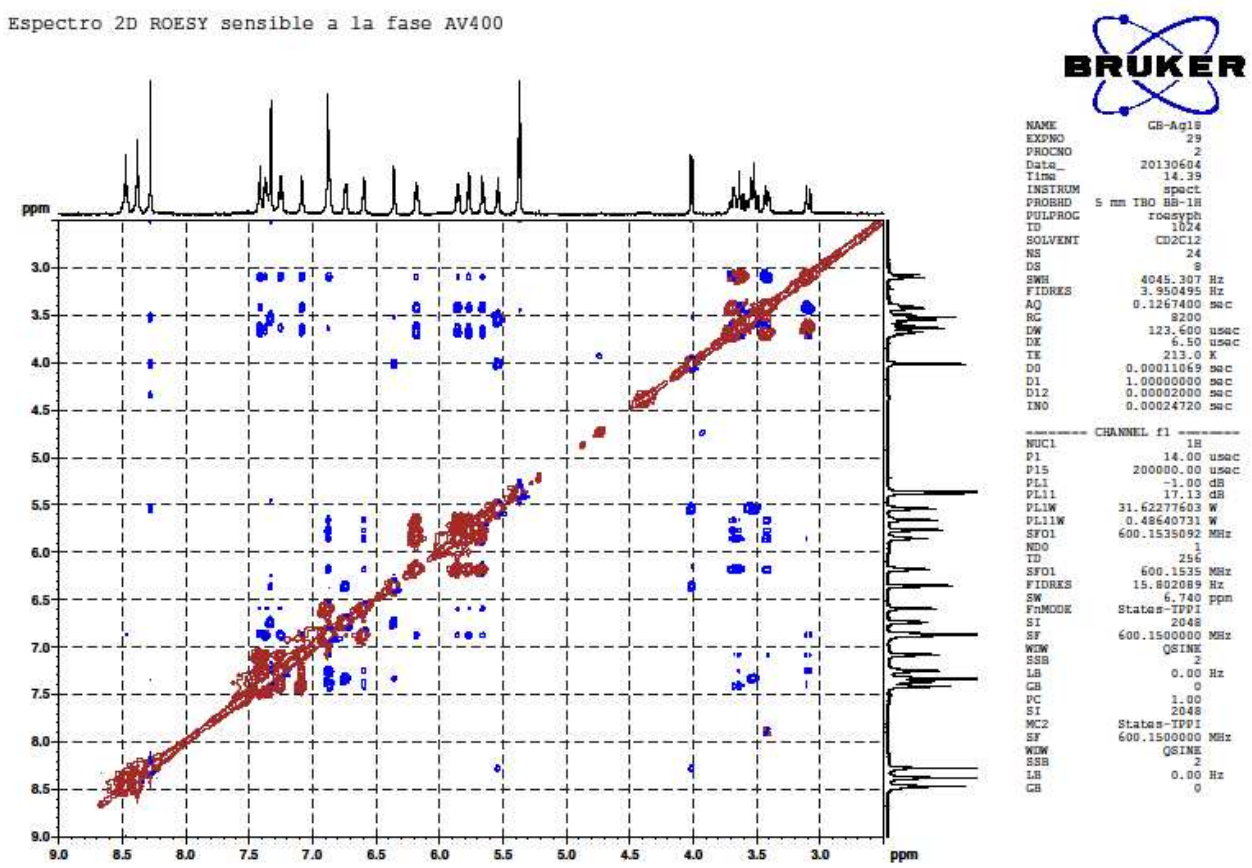

Complex 12. 2D ROESY 213K
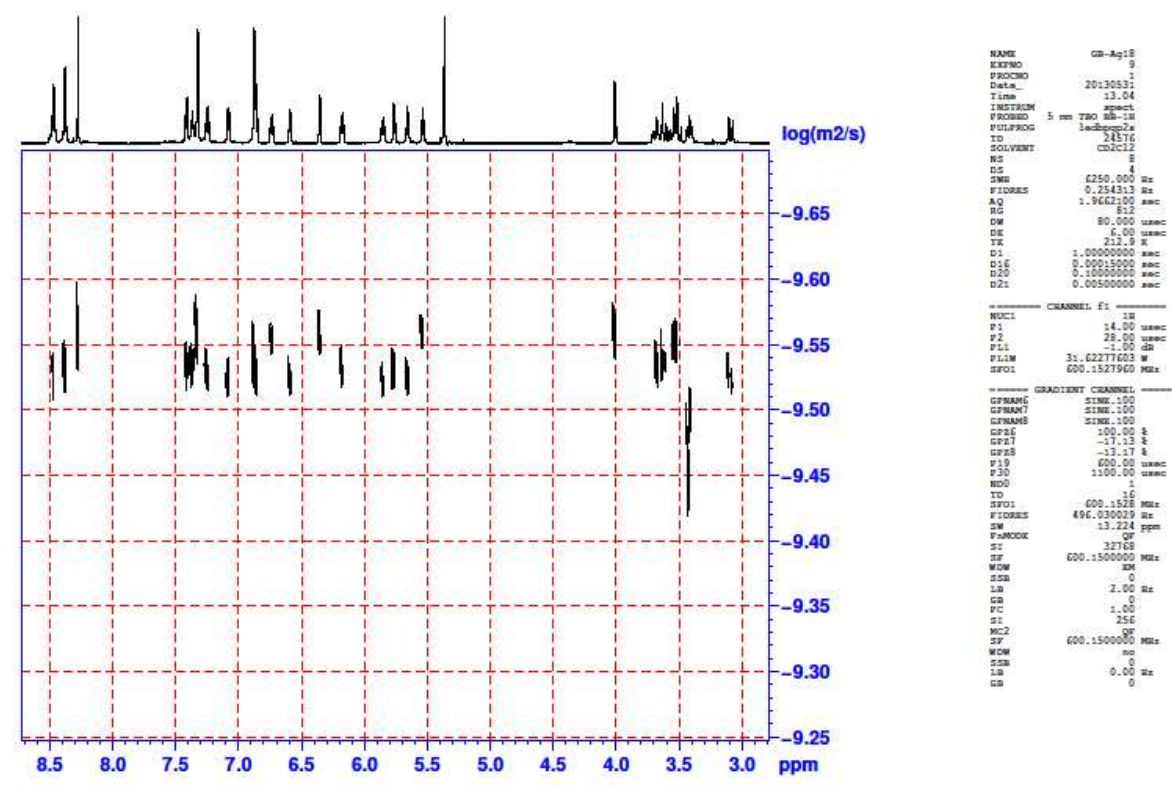

Complex 12. ${ }^{1} \mathrm{H}$ DOSY $213 \mathrm{~K}$ (logarithmic scale) 

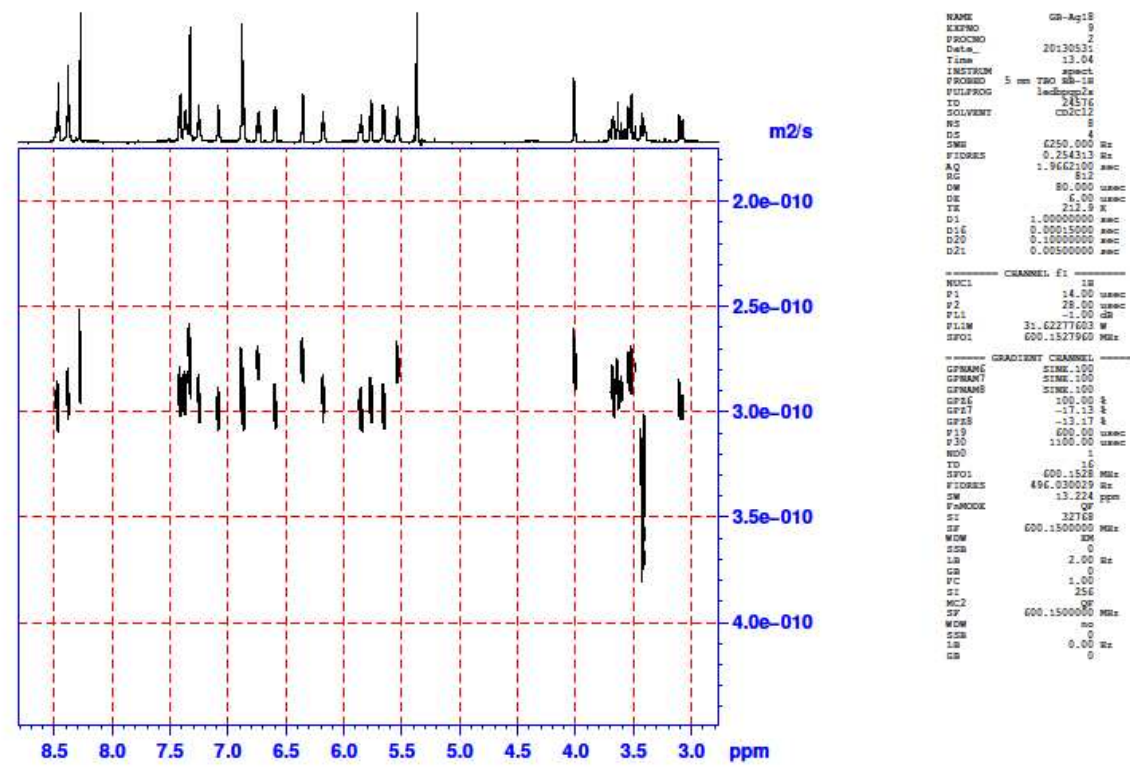

Complex 12. ${ }^{1} \mathrm{H}$ DOSY $213 \mathrm{~K}$ (linear scale) 